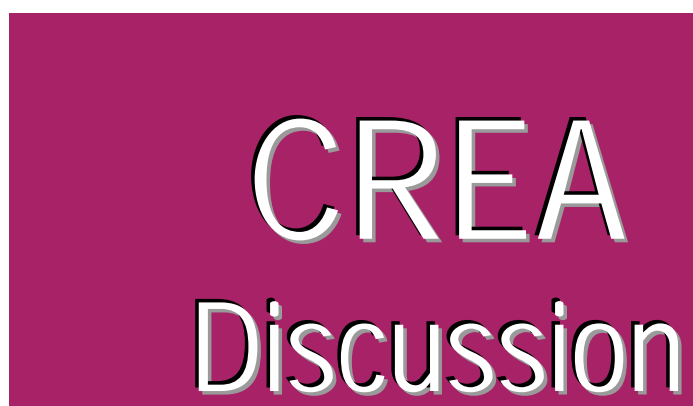
Paper 2015-12 Economics Center for Research in Economics and Management University of Luxembourg

\title{
Currency Areas and Voluntary Transfers
}

available online : http://wwwfr.uni./u/recherche/fdef/crea/publications2/discussion_papers

Pierre M. Picard, CREA, Université du Luxembourg Tim Worrall, University of Edinburgh

May, 2015

For editorial correspondence, please contact: crea@uni.lu University of Luxembourg Faculty of Law, Economics and Finance 162A, avenue de la Faïencerie L-1511 Luxembourg 


\title{
Currency Areas and Voluntary Transfers*
}

\author{
By Pierre M. Picard ${ }^{\dagger}$ and Tim Worrall ${ }^{\ddagger}$
}

May 2015

\begin{abstract}
This paper discusses the relationship between the formation of a currency area and the use of voluntary fiscal transfers between countries. We show that there is a trade off between the benefits of flexible exchange rates and the additional risk sharing benefits of voluntary transfers that can be sustained in a currency area. We show that whether a currency area is beneficial or not will depend on the magnitude of economic parameter values. In particular, we show that in a simple two country model and for a plausible set of economic parameter values, a currency area is optimal.
\end{abstract}

\section{Introduction}

The recent controversy over the refinancing of high deficit countries within the Euro-zone has highlighted the difficulty of adopting a common fiscal policy amongst sovereign states. The reluctance of the core Euro-zone countries to provide fiscal assistance to peripheral countries has not only renewed the debate about whether the Euro-zone is an optimal currency area but also has cast doubt about whether it might become so. ${ }^{1}$

This paper considers how the possibility of voluntary fiscal transfers between sovereign nations affects the case for/or against optimal currency areas. Our analysis is based on two fundamental ideas. First, that there are welfare gains to risk sharing between countries. There is much evidence to support this position. Amongst others, Forni and Reichlin (1999) have shown that there exists a large potential insurable income risk in the E.U. (about 45\%) and that risk diversification is highly

*We thank Michael Artis, Jacques Drèze, Paul De Grauwe and Jacques Melitz for fruitful discussions. The second author gratefully acknowledges the support of the Hallsworth Research Fellowship Fund at the University of Manchester. All errors are ours.

${ }^{\dagger}$ CREA, University of Luxembourg, 162A avenue de la Faïencerie, L-1511 Luxembourg and CORE, Université Catholique de Louvain, Louvain-la-Neuve, Belgium.

${ }^{\ddagger}$ School of Economics, University of Edinburgh, 30 Buccleuch Place, Edinburgh, EH8 9JT, UK.

${ }^{1}$ Fiscal integration has long been recognized as an important issue in the setting up of a currency union (see, e.g., Kenen, 1969). 
incomplete. ${ }^{2}$ Second, any transfer promises are not contractually enforceable and a country will only make a transfer if it perceives that the long-term benefit of risk sharing offsets the current cost of making the transfer. That is, all transfers must be voluntary.

The key question we study is whether or not a currency area can sustain greater transfers than the flexible exchange rate regime and the extent to which such risk sharing benefits offset the loss of the ability to depreciate the currency, when there is an adverse shock combined with a sticky wage, that the flexible exchange rate regime offers. To examine this issue, we consider a two-country, open economy model with shocks to labor productivity. In much of the analysis, we assume that crosscountry productivity is negatively correlated to ensure that the demand for risk-sharing is large. Households consume differentiated products and money balances and supply their labor to pricesetting producers. They are monopsonists in their supply of labor. A wage rigidity is introduced by assuming that wages are set in advance, before the productivity is known. We consider two cases: a common currency area in which the exchange rate is fixed exogenously, and a flexible exchange rate regime. We embed this model in a repeated context and allow countries to make voluntary transfers contingent on productivity shocks. Transfers are voluntary in the sense that the long term benefits of making a transfer exceed the short term costs. Transfers are sustained by the threat of returning to a situation of no transfers. To keep the model tractable, we assume there are no mechanisms other than transfers to smooth consumption across time. This is a strong assumption, but it is a good starting point given the evidence that risk diversification is highly incomplete. ${ }^{3}$

It is important to note that our baseline model is set up to embed three features that are usually considered inimical to an optimum currency area (Mundell, 1961). That is, there is wage stickiness in the labor market, asynchronous business cycles and absence of transaction costs. ${ }^{4}$ The asynchronicity of productivity shocks exacerbates the negative impact of wage stickiness on labor market adjustments and consumption. Similarly, the absence of transaction costs means that a common currency area does not have the benefit of reducing transaction costs. ${ }^{5}$ However, the asynchronicity of productivity shocks and wage stickiness increase the benefits of voluntary transfers. If a currency area is associated with greater risk-sharing, then the benefits of a currency area may outweigh its costs, reversing Mundell's result. The contribution of this paper is to provide a model in which these issues can be examined and provide conditions where Mundell's result is reversed.

Our results show that for a plausible set of parameter values, a currency area can be optimal. The reason for this result is that the currency area sustains more risk sharing through voluntary transfers than does a flexible exchange rate regime. A currency area can sustain more risk-sharing

\footnotetext{
${ }^{2}$ See also French and Poterba (1991), Baxter and Jermann (1997) and Lewis (1999).

${ }^{3}$ Given our assumption that there are no financial markets that share risk across countries, there are no financial transactions to cause the current account to fluctuate and therefore, the nominal exchange rate is determined through a balanced trade condition in the flexible exchange rate regime.

${ }^{4}$ We do consider the implications of transaction costs in Section 4.

${ }^{5}$ For an analysis, see, for example, Mundell (1961), Mundell (1973), Bayoumi (1994) and Alesina and Barro (2002).
} 
because the same productivity shocks create a larger variance in consumption in a currency areas while the cost of reneging on the tacitly agreed transfers is higher in a currency area than in the flexible exchange rate regime. In this sense, the formation of a currency area is a commitment device that may allow countries to share more risk.

The currency area achieves better risk sharing, but only for some parameter values. When the desire for risk sharing is large and the future is not discounted too heavily, full risk sharing can be achieved in both regimes and the flexible exchange rate regime will dominate. Equally, when the desire for risk sharing is low and the future more heavily discounted, no risk sharing can be sustained in either regime and again the flexible exchange rate regime will dominate. However, for intermediate values the currency area may sustain more risk sharing and the benefits of this extra risk sharing may offset the advantages of a flexible exchange rate to adjust to productivity differences. The analysis is complicated because risk sharing has indirect effects through the exchange rate and through the wage that is set in advance in anticipation of the risk sharing transfers. However, in a two state case, we can show analytically that for some set of parameter values, consumption sharing is sustained in the currency area whereas no transfers can be sustained under flexible exchange rates, and that the currency area dominates. We also show that the currency area can dominate even when productivity shocks are small. In a numerical analysis, we show that the currency area can dominate for some plausible parameter values. The key parameters we examine are risk aversion, labor supply elasticity, the elasticity of substitution between goods, the size of the productivity shock and the discount factor.

Our analysis shows that the choice of a currency regime cannot be disentangled from choices about risk sharing. It suggests that currency areas may, in some circumstances, make redistribution more likely. Empirically, this may be hard to establish. Rose and Engel (2002) find a small but positive relationship between currency areas and risk sharing, but given limited data, the effect is statistically insignificant. Of course, long-lived currency areas with a federal structure such as the U.S.A. and Germany have had considerable intra-country risk sharing. It will be interesting to see if in future the members of the Euro-zone countries establish greater risk sharing between themselves compared to other E.U. member states.

Related literature The paper relates to the literature on optimum currency areas and risk sharing initiated by Mundell (1973). Kenen (1969) emphasizes the need for interregional transfers within a currency area and Drèze (2000) demonstrates that transfers between regions can be used as a means of insurance against regional income shocks. ${ }^{6}$ We build, in particular, upon the analysis of

\footnotetext{
${ }^{6}$ Cooper and Kempf (2004) examine a slightly different trade-off. They have risk sharing within countries but no transfers between countries. In their model a currency union overcomes a cash in advance constraint, allowing consumption to be adapted to taste shocks, but the central monetary authority in a currency union cannot adapt its policy to divergent unemployment shocks in the separate countries. Thus, a currency union is welfare improving when unemployment shocks are sufficiently positively correlated across countries and when taste shocks are sufficiently
} 
Devereux (2004) and Ching and Devereux (2003). Devereux (2004) considers a static model with incomplete financial markets and no risk sharing. He demonstrates that a currency area can be desirable because, absent financial markets, both regimes produce inefficient outcomes and the fixed exchange rate can be associated with more stability. A key parameter in his analysis is the elasticity of labor supply: when labor supply is very elastic, a currency area dominates because output becomes highly responsive to demand shocks in a direction that would be chosen by a social planner. Ching and Devereux (2003) consider a similar model where risk is fully shared in the common currency environment but where no risk is shared in the flexible exchange rate regime. Our baseline model is similar to Devereux (2004) except that in our model countries produce differentiated products and there are productivity rather than preference shocks. However, we consider a repeated version of the model and endogenize risk-sharing transfers by considering transfers that maximize welfare subject to self-enforcing constraints.

Our model is also in the tradition of the New Open Economy Macroeconomics. This literature has mostly focused on monetary policies under the assumption of complete financial markets (see, e.g., Obstfeld and Rogoff, 1995; Corsetti et al., 2010). Under this assumption, financial markets offer an important risk sharing mechanism so that fiscal transfers between countries are not likely to relevant. However, a number of papers have considered cases where financial markets are incomplete, (see, e.g., Corsetti et al., 2008). Our analysis is in this vein. However, we assume that there are no financial markets and that transfers must be used to share risk. Furthermore, we suppose that the ability to share risk through transfers is limited by the willingness of countries to voluntary engage in such mechanisms.

Four related papers that address currency unions in a similar context to ours are Arellano and Heathcote (2010), Castro and Koumtingué (2014), Farhi and Werning (2014) and Fuchs and Lippi (2006). Arellano and Heathcote (2010) consider full dollarization rather than a currency union and non-contingent debt rather than risk sharing. The basic mechanism at work is however, similar: borrowing is limited because default is punished only by exclusion from future borrowing; dollarization has a cost because there is a loss in seigniorage but the very fact that counties cannot use monetary easing makes the costs of default on borrowing greater and hence may allow the country to borrow more in international markets. Castro and Koumtingué (2014) also considers risk sharing and limited commitment in examining the optimality of a currency union. They however, assume that the formation of a union enables full risk sharing and that trade with countries outside of the union is restricted by limited enforcement. Thus, their modeling assumptions are very different from ours. Farhi and Werning (2014) address a similar issue but in a different model. Their model has a non-traded good and sticky prices that generate an aggregate demand externality. In their dynamic model, financial markets are incomplete, but shocks occur only once at the beginning of the first period. In our model, the need for transfers arises because shocks are repeated. Fuchs and Lippi large. 
(2006) consider a dynamic policy game where policy has to be coordinated in a monetary union. This provides a tension between co-ordination and flexibility. Although Fuchs and Lippi (2006) consider the temporal incentives to leave the monetary union, they do not provide a welfare analysis of the two regimes.

The paper is organized as it follows. Section 2 presents the baseline model of the two different currency regimes when there are transfers between countries. Section 3 considers the sustainability of transfers between countries. That is, it considers transfers where the short term cost of the transfer is offset by future risk sharing benefits. Section 4 extends the analysis to allow for transaction costs. Section 5 concludes. Proofs and detailed derivations are contained in the Appendices.

\section{Model}

In this section we present a standard one-period, two-country open-economy model with money demand. Labor productivity is different across countries and uncertain. Households are monopsonists in their supply of labor. A price rigidity is introduced by assuming households set wages in advance, before productivity is known. Countries can make transfers to one another, contingent on the productivity shock, so as to share risk. We consider both the case of a currency area where the exchange rate is exogenously fixed, and a flexible exchange rate regime. Transfers will affect wage setting in both scenarios and will also affect the exchange rate in the flexible exchange rate regime. At the end of the section, we consider the equilibrium outcome when transfers equalize consumption across countries. Section 3 then considers a repeated version of the model in which transfers are voluntary, that is, are only made if the long run benefits of future risk sharing exceed the short run costs of making a transfer.

\section{The set up}

There are two countries, Home and Foreign. Both countries have a unit mass of domestic households and a unit mass of firms producing differentiated tradeable varieties. Countries are identical except that they may experience different realizations of a productivity shock. The exposition of the model

focuses on Home, with analogous expressions holding for Foreign. Foreign variables are denoted with a star.

Demands The representative Home household $h \in[0,1]$ has preferences given by a utility function

$$
U(h):=V\left((c(h))^{\mu}\left(\frac{m(h)}{P}\right)^{1-\mu}\right)-\chi \frac{\ell(h)^{1+\psi}}{1+\psi}
$$


where

$$
c(h)=\left(\int_{0}^{1}(d(v, h))^{\rho} \mathrm{d} v+\int_{0}^{1}\left(d\left(v^{*}, h\right)\right)^{\rho} \mathrm{d} v^{*}\right)^{1 / \rho}
$$

is the composite of the consumption of local varieties $v \in[0,1]$ (denoted $d(v, h)$ ) and foreign varieties $v^{*} \in[0,1]$ (denoted $\left.d\left(v^{*}, h\right)\right), m(h) / P$ is the real money balance or cash holdings and $\ell(h)$ is the household's labor supply (worked hours). The parameter $\psi \geq 0$ is the inverse of the Frisch elasticity of labor supply. ${ }^{7}$ The parameter $\mu \in(0,1)$ denotes the preference and share of expenditure for real money balances. The parameter $\rho \in(0,1)$ is the intensity of the preference for product variety. The elasticity of substitution between product varieties, $\sigma>1$, is positively related to $\rho$ : $\sigma=1 /(1-\rho)$. The function $V$ is assumed to exhibit constant relative risk aversion, with $V(x):=x^{1-\gamma} /(1-\gamma)$ for $\gamma>1$, where $x$ denotes the composite of the consumption of varieties and real money balances. For simplicity, we refer to $x$ as consumption. Finally, the parameter $\chi$ represents the intensity of preference for leisure. In our model, the parameter $\chi$ plays no role and it is set equal to one in the rest of the paper.

The Home household $h$ maximizes its utility subject to the budget constraint

$$
\int_{0}^{1} p(v) d(v, h) \mathrm{d} v+\int_{0}^{1} p\left(v^{*}\right) d\left(v^{*}, h\right) \mathrm{d} v^{*}+m(h)=y(h)+T+M_{0}
$$

where $p(v)$ is the Home price of domestic variety $v, p\left(v^{*}\right)$ is the Home price of Foreign variety $v^{*}, y(h)$ is the income of household $h, T$ is a transfer received by the Home country (the aggregate transfer $T$ is distributed equally to all households) and $M_{0}$ is the endowment of local money (distributed equally to all households). Household income has two components: labor income $w(h) \ell(h)$, where $w(h)$ is the household wage (see below), and an equal share of local profits, $\Pi$. That is, household income is $y(h)=w(h) \ell(h)+\Pi$. Money is the unit of numéraire and is exogenously supplied by the country's central bank. For simplicity, money fully depreciates in each time period. In the above expression, all values are denominated in the Home country's currency.

Under this specification, it is well known that the households maintain a constant expenditure share for each type of good. So, $c(h)$ and $m(h)$ satisfy

$$
\frac{P c(h)}{\mu}=\frac{m(h)}{1-\mu}=w(h) \ell(h)+\Pi+T+M_{0},
$$

where

$$
P:=\left(\int_{0}^{1}(p(v))^{1-\sigma} \mathrm{d} v+\int_{0}^{1}\left(p\left(v^{*}\right)\right)^{1-\sigma} \mathrm{d} v^{*}\right)^{\frac{1}{1-\sigma}}
$$

is the Home price index. The demand for the local variety $v$ by household $h$ is

$$
d(v, h)=\mu \frac{(p(v))^{-\sigma}}{P^{1-\sigma}}\left(w(h) \ell(h)+\Pi+T+M_{0}\right)
$$

\footnotetext{
${ }^{7}$ If $\psi=0$, then labor supply is infinitely elastic.
} 
while its demand for the imported variety $d\left(v^{*}, h\right)$ is defined similarly with $p\left(v^{*}\right)$ replacing $p(v)$. Hence, consumption for household $h$ is $x(h):=c(h)^{\mu}(m(h) / P)^{1-\mu}=\xi\left(w(h) \ell(h)+\Pi+T+M_{0}\right) / P$ where $\xi:=\mu^{\mu}(1-\mu)^{1-\mu}$.

We define the nominal exchange rate, $\varepsilon$, as the units of Home currency required to purchase one unit of Foreign currency. ${ }^{8}$ With no transaction costs (Section 4 below considers the implications of transaction costs), $p(v)=\varepsilon p^{*}(v)$ and $p\left(v^{*}\right)=\varepsilon p^{*}\left(v^{*}\right)$. Using equation (1), it is easy to check that $P=\varepsilon P^{*}$. Also, since there are no transfers from outside the two countries, we have $T=-\varepsilon T^{*}$. Denoting the aggregate of the local income as $Y:=\int_{0}^{1} y(h) \mathrm{d} h$, total world income, denominated in the Home currency, is $Y^{w}:=Y+\varepsilon Y^{*}$. Similarly, the world money supply in terms of the Home currency is $M_{0}^{w}:=M_{0}+\varepsilon M_{0}^{*}$. The aggregate demand for local and foreign varieties $v$ and $v^{*}$, are defined as $D(v):=\int_{0}^{1} d(v, h) \mathrm{d} h$ and $D\left(v^{*}\right):=\int_{0}^{1} d\left(v^{*}, h\right) \mathrm{d} h$. Given the above analysis, they can be written as

$$
D(v)=\mu \frac{(p(v))^{-\sigma}}{P^{1-\sigma}}\left(Y^{w}+M_{0}^{w}\right) \quad \text { and } \quad D\left(v^{*}\right)=\mu \frac{p^{*}\left(v^{*}\right)^{-\sigma}}{P^{1-\sigma}} \frac{\left(Y^{w}+M_{0}^{w}\right)}{\varepsilon} .
$$

If the two countries belong to a common currency area, then $\varepsilon=1$.

Production Home firms hire imperfectly substitutable labor services from local households and are price setters in their product market. Consider a Home firm producing the variety $v$. The firm hires $\ell(v, h)$ units of differentiated labor service from each local household $h$ to produce an amount of output given by the production function

$$
F(\ell(v, \cdot)):=\frac{1}{a}\left(\int_{0}^{1}(\ell(v, h))^{\frac{\theta-1}{\theta}} \mathrm{d} h\right)^{\frac{\theta}{\theta-1}}
$$

where $\theta>1$ measures the elasticity of substitution between labor services and $1 / a$ is the country productivity. A lower $\theta$ is associated with more differentiated labor services, which gives households more power in wage setting. Therefore, a lower $\theta$ implies a higher mark-up over the marginal disutility of labor. An increase in $1 / a$ implies a fall in the labor input needed for each unit of output.

The firm chooses the labor input mix $\ell(v, \cdot)$ that minimizes its cost per unit of output $\int_{0}^{1} w(h) \ell(v, h) \mathrm{d} h$ subject to $F(\ell(v, \cdot))=1$. Its demand for labor service per unit of output can therefore be computed as

$$
\ell(v, h)=a\left(\frac{w(h)}{W}\right)^{-\theta}
$$

where

$$
W:=\left(\int_{0}^{1}(w(h))^{1-\theta} \mathrm{d} h\right)^{1 /(1-\theta)}
$$

is the Home wage index. As a consequence, the cost per unit of output equals $a W$ and the firm's profit is:

$$
\pi(v)=(p(v)-a W) D(v) .
$$

\footnotetext{
${ }^{8}$ So an appreciation of the Home currency corresponds to a fall in $\varepsilon$.
} 
The firm chooses the price $p(v)$ to maximize $\pi(v)$ taking exchange rates and prices indices as given. Because its demand is iso-elastic, it sets the price $p(v)=a W / \rho$, which is the same for each variety. Letting $p$ denote the common price, there is a common profit, denoted by $\pi$. Moreover, the demand for each variety is the same, and we denote this common demand by $D$. With a unit mass of firms, profits $\pi$ equals the aggregate profit $\Pi$.

Labor market The labor market is the source of rigidity in this model. As will be explained below the local productivity, $1 / a$ is uncertain and workers set their wages before the realization $a$ is known, adapting their labor supply to balance the demand of local firms at the preset wage after the productivity parameter is realized. For the moment, suppose that all wages $w(h)$ are pre-set and consider a Home household $h$. At its pre-set wage, $w(h)$, the household matches its labor supply to the labor demand by each firm. Each firm's labor demand is equal to its output $D(v)=D$ times its labor input per unit of output given in equation (2). Aggregating over all local firms, gives the total demand to the household $\ell(h):=\int_{0}^{1} \ell(v, h) \mathrm{d} v$, which simplifies as

$$
\ell(h)=a\left(\frac{w(h)}{W}\right)^{-\theta} D .
$$

Furthermore, because of domestic firms and households are symmetric and face the same local economic conditions, Home households must have the same wage and offer the same supply of labor to firms: $w(h)=W$ and $\ell(h, v)=\ell(h)=: \ell$. Since households have a unit mass, the aggregate labor supply is equal to $L:=\int_{0}^{1} \ell(h) \mathrm{d} h=\ell$. Equally, consumption and earnings are the same for all households and the aggregate consumptions and incomes are given by $C:=\int_{0}^{1} c(h) \mathrm{d} h, M:=\int_{0}^{1} m(h) \mathrm{d} h$, $X:=\int_{0}^{1} x(h) \mathrm{d} h$, and $Y:=\int_{0}^{1} y(h) \mathrm{d} h$. Likewise, all firms in the same country set the same prices, hire the same amount of labor, and produce the same quantity of each variety. Hence, the indices $h$ and $v$ referring to households and firms can be dropped from now on.

Equilibrium Now we consider the equilibrium relationships for given inverse productivity $a$, transfer $T$, wages $W$ (and $W^{*}$ ) and exchange rate $\varepsilon$. The model will be closed by the determination of the exchange rate when we consider the currency area and flexible exchange rate regimes below. ${ }^{9}$ Product prices are set by firms such that

$$
p=a \frac{W}{\rho} \quad \text { and } \quad p^{*}=a^{*} \frac{W^{*}}{\rho} .
$$

Thus, $p=\left(a / a^{*}\right)\left(W / W^{*}\right) p^{*}$. Labor supply clears with its demand

$$
L=a D .
$$

\footnotetext{
${ }^{9}$ We consider wage setting shortly and we will consider the determination of transfers in the next Section.
} 
National income equals the value of production

$$
Y=W L+\Pi=p D=\frac{W}{\rho} L
$$

The demand for goods and money holding are proportional to disposable income

$$
\frac{P C}{\mu}=\frac{M}{1-\mu}=Y+T+M_{0}
$$

where $P$ is the local price index. World money demand equals world money supply: $M+\varepsilon M^{*}=$ : $M^{w}=M_{0}^{w}$. In this model, we can, w.l.o.g., normalize the money supplies to $M_{0}=M_{0}^{*}=(1-\mu) / \mu{ }^{10}$ Thus, using (7) and the fact that $T+\varepsilon T^{*}=0$, it follows that $Y^{w}+M_{0}^{w}=M^{w} /(1-\mu)=(1+\varepsilon) / \mu$ and $Y^{w}=(1+\varepsilon)$. Thus, the demand for each variety are equal to

$$
D=\frac{p^{-\sigma}}{P^{1-\sigma}}(1+\varepsilon) \quad \text { and } \quad D^{*}=\frac{\left(p^{*}\right)^{-\sigma}}{\left(P^{*}\right)^{1-\sigma}} \frac{(1+\varepsilon)}{\varepsilon} .
$$

The local price index is given by

$$
P(W, \varepsilon):=(W / \rho)\left((a)^{1-\sigma}+\left(\varepsilon \frac{W^{*}}{W}\right)^{1-\sigma}\left(a^{*}\right)^{1-\sigma}\right)^{\frac{1}{1-\sigma}}
$$

where $W /\left(\varepsilon W^{*}\right)$ is the relative wage. To discuss the role of exchange rates, it is convenient to disentangle the effect of global productivity from local terms of trade. Towards this aim, we re-write the local price index more succinctly as

$$
P(W, \varepsilon)=(W / \rho) A B(\varepsilon)
$$

where

$$
A:=\left(a^{1-\sigma}+\left(a^{*}\right)^{1-\sigma}\right)^{\frac{1}{1-\sigma}} ; \quad b:=\frac{a^{1-\sigma}}{\frac{1}{2} A^{1-\sigma}} ; \quad B(\varepsilon):=\left(\frac{1}{2} b+\frac{1}{2} b^{*}\left(\varepsilon \frac{W^{*}}{W}\right)^{1-\sigma}\right)^{\frac{1}{1-\sigma}}
$$

The term $A$ measures global productivity, $b$ is a measure of relative productivity and $B(\varepsilon)$ embeds the effect of relative wages, $\varepsilon W^{*} / W$, on local prices. ${ }^{11}$ When $\varepsilon W^{*} / W=1, B(1)=b / 2+b^{*} / 2=1$, and local prices do not depend on the relative wage. ${ }^{12}$

\footnotetext{
${ }^{10}$ With the wage setting described below, the level of money supply is irrelevant in this model: a proportional rise in money supply will be matched by the same proportional rise in wages and prices. With this normalization, money supplies exactly adjust to households' preferences for cash holdings so that any changes in the preferences for cash holdings, $\mu$, is accompanied with a rise in the money supply that keeps nominal prices and wages unchanged.

${ }^{11}$ Since $b / 2+b^{*} / 2=1, B(\varepsilon)$ is a weighted power mean of 1 and $\varepsilon\left(W^{*} / W\right)$ with weights $b / 2$ and $b^{*} / 2$. Thus, $\min \left\{1, \varepsilon\left(W^{*} / W\right)\right\}<B(\varepsilon)<\left(\varepsilon\left(W^{*} / W\right)\right)^{\left(b^{*} / 2\right)}$ where the second inequality follows because $\sigma>1$ implies $B(\varepsilon)$ is less than the weighted geometric mean.

${ }^{12}$ Since $P=\varepsilon P^{*}$, we have $B^{*}(\varepsilon)=\frac{W}{\varepsilon W^{*}} B(\varepsilon)$.
} 
Taking the ratio of the expressions in (8), using (4) and (5) and $P=\varepsilon P^{*}$ allows us to express the exchange rate as

$$
\varepsilon=\left(\frac{W}{W^{*}}\right)\left(\frac{b}{b^{*}}\right)^{-\frac{1}{\sigma}}\left(\frac{L}{L^{*}}\right)^{\frac{1}{\sigma}}
$$

From this equation, it can be seen that for fixed wages, changes in $b / b^{*}$ can be absorbed either through changes in the exchange rate or changes in labor supplies (and thus, production). Letting $L(W, \varepsilon)$ denote labor supply as a function of the wage and exchange rate and $Y(\varepsilon)$ denote national income, we have

$$
L(W, \varepsilon)=b \frac{\rho}{W} \frac{\frac{1}{2}(1+\varepsilon)}{B(\varepsilon)^{1-\sigma}} ; \quad Y(\varepsilon):=W L(W, \varepsilon) / \rho=b \frac{\frac{1}{2}(1+\varepsilon)}{B(\varepsilon)^{1-\sigma}},
$$

where $Y(\varepsilon)$ is independent of wage if $W=W^{*}$. Also note that if $W=W^{*}$, then $Y(1)=b$ since $B(1)=1$. Money demand can be written as $M(\varepsilon, T)=(1-\mu)\left(Y(\varepsilon)+T+M_{0}\right)$ and consumption $X(T, W, \varepsilon)=\xi\left(Y(\varepsilon)+T+M_{0}\right) / P(W, \varepsilon)$. Finally, utility can be written as

$$
U(T, W, \varepsilon)=V(X(T, W, \varepsilon))-\frac{L(W, \varepsilon)^{1+\psi}}{1+\psi} .
$$

So, we have described the economy for a given set of productivity parameters $\left(a, a^{*}\right)$. We now make precise the structure of productivity shock uncertainty and describe the wage setting.

Uncertainty and symmetry The only uncertainty in the model is a shock to a country's productivity. Thus, we treat the inverse productivities $a$ and $a^{*}$ as random variables. Each state of nature $s \in S$ is therefore defined by pair of inverse productivity levels $\left(a_{s}, a_{s}^{*}\right)$ for Home and Foreign. We will use the subscript $s$ where we wish to emphasize state dependence. For example, we denote the transfer in state $s$ by $T_{s}$, the exchange rate in state $s$ by $\varepsilon_{s}$ and so on.

From the above model description, it is clear that the two countries are structurally symmetric because they face the common parameters $\mu, \psi, \rho, \gamma$, and $\theta$. We assume additional symmetry by imposing symmetric probability distributions of country productivity shocks. That is, for every state $s$ with inverse productivity pair $\left(a_{s}, a_{s}^{*}\right)$, we assume that there exists another state $q$ with the same probability and the opposite shock structure: $\left(a_{q}, a_{q}^{*}\right)=\left(a_{s}^{*}, a_{s}\right)$. Thus, the two countries are ex-ante symmetric and households form the same expectations in both countries.

Wage Setting We now explain how the wage is determined. We assume that workers set their wage before the shocks to productivities are realized. Hence, wages are sticky and do not respond to productivity shocks. Home household $h$ sets its wage $w(h)$, considering its own labor demand (3) and taking aggregate profits $\Pi_{s}$, transfers $T_{s}$, prices $P_{s}$ for $s \in S$ and money supply $M_{0}$ as given, to maximize its expected utility

$$
\mathrm{E}\left[U_{s}(h)\right]=\mathrm{E}\left[V\left(\xi \frac{w(h) \ell_{s}(h)+\Pi_{s}+T_{s}+M_{0}}{P_{s}}\right)\right]-\frac{\mathrm{E}\left[\ell_{s}(h)^{1+\psi}\right]}{1+\psi},
$$


where $\mathrm{E}$ is the expectation over all states $s \in S$. Aggregating over the household's first-order conditions, it can be checked (see Appendix A) that for a given set of transfers, $T_{s}$ and given set of exchange rates, $\varepsilon_{s}$, the wage satisfies ${ }^{13}$

$$
W=\frac{\theta}{\theta-1} \frac{\mathrm{E}\left[L_{s}\left(W, \varepsilon_{s}\right)^{1+\psi}\right]}{\mathrm{E}\left[\xi V^{\prime}\left(X_{s}\left(T_{s}, W, \varepsilon_{s}\right)\right) \frac{L_{s}\left(W, \varepsilon_{s}\right)}{P_{s}\left(W, \varepsilon_{s}\right)}\right]} .
$$

We are now equipped to discuss the impact of of the exchange rate system on the households' utility.

\subsection{Currency area}

We first consider the equilibrium in which the two countries belong to a common currency area, where $\varepsilon_{s}=1$ for all $s \in S$. World income is constant, $Y_{s}^{w}=\left(1+\varepsilon_{s}\right)=2$ for each $s \in S$. Since countries are ex-ante symmetric, households in the two countries set the same wages: $W=W^{*}$. With $W=W^{*}, B_{s}(1)=1$ and therefore, price indices depend on the wage $W$ with

$$
P_{s}(W):=P_{s}(W, 1)=\left(\frac{W}{\rho}\right) A_{s} .
$$

Prices are equal in both countries, $P_{s}^{*}(W)=P_{s}(W)$. Likewise, employment is given by

$$
L_{s}(W):=L_{s}(W, 1)=b_{s} \frac{\rho}{W} .
$$

The higher relative productivity country (higher $b_{s}$ ) has a higher employment level. National income is equal to relative productivity: $Y_{s}(1)=b_{s}$, and is independent of transfers. Employment is only indirectly affected by transfers through the wage. This indirect effect of transfers changes the level but not the variability of employment because wages are fixed in advance of the productivity shocks. Money demand in state $s$ however, depends on the transfer in that state: $M_{s}\left(T_{s}\right):=(1-\mu)\left(b_{s}+\right.$ $T_{s}+M_{0}$ ). Using the wage set in (4) and the labor supply (12), one can verify (see Appendix A) that the wage $W$ satisfies

$$
W=\left(\kappa_{0} \frac{\mathrm{E}\left[b_{s}^{1+\psi}\right]}{\mathrm{E}\left[\left(\mu\left(b_{s}+T_{s}\right)+(1-\mu)\right)^{-\gamma} b_{s} A_{s}^{\gamma-1}\right]}\right)^{\frac{1}{\gamma+\psi}}
$$

where $\kappa_{0} \equiv \rho^{\gamma+\psi-1} \xi^{\gamma-1} \mu^{-\gamma} \theta /(\theta-1)$. We denote by $W_{0}$ the wage set in the absence of any transfers $\left(T_{s}=0\right.$ for each state $\left.s\right)$.

Utility in the Home country in state $s$ as a function of the wage the transfer $T_{s}$ in that state and the wage $W$ is given by

$$
U_{s}\left(T_{s}, W\right):=U_{s}\left(T_{s}, W, 1\right)=V\left(X_{s}\left(T_{s}, W\right)\right)-\frac{\left(L_{s}(W)\right)^{1+\psi}}{1+\psi},
$$

\footnotetext{
${ }^{13}$ We leave implicit the dependence of $W$ on the vector of transfers for the moment. The effect of an increase in risk sharing on the wage set depends on the effect on expected marginal utility. Although an increase in risk sharing will tend to reduce the variability in marginal utility, the effect on expected marginal utility is, in general, ambiguous.
} 
where $X_{s}\left(T_{s}, W\right)=\xi\left(b_{s}+T_{s}+M_{0}\right) / P_{s}(W)$. Utility $U_{s}\left(T_{s}, W\right)$ is an increasing and concave function of the received transfer $T_{s}$. The effect of a change in the wage on utility is ambiguous because an increase in $W$ reduces employment, which is good for utility, but also increases prices and reduces consumption.

\section{$2.2 \quad$ Flexible exchange rate}

We now consider equilibrium output and consumption under a flexible exchange rate regime. A flexible exchange rate provides an additional instrument through which relative prices can adjust to lessen the impact of shocks on production and employment. Under a flexible exchange rate regime, money demand equates its supply within each country, so that $M=M^{*}=M_{0}=(1-\mu) / \mu$. Again, since countries are ex-ante symmetric, households set the same ex-ante wages, $W=W^{*}$. From (7), $P_{s} C_{s}=1$ for each state $s$, and therefore using $M_{0}=(1-\mu) / \mu$, national income is $Y_{s}\left(\varepsilon_{s}\right)=1-T_{s}$. A transfer is completely offset by a reduction in national income. Using the definition of $Y_{s}\left(\varepsilon_{s}\right)$ in $(2)$ and the condition $Y_{s}\left(\varepsilon_{s}\right)=1-T_{s}$, the exchange rate can be determined implicitly as a function of transfer in that state. That is, $\varepsilon_{s}\left(T_{s}\right)$ is implicitly defined by $b_{s}\left(1+\varepsilon_{s}\right)=2\left(1-T_{s}\right) B_{s}\left(\varepsilon_{s}\right)^{1-\sigma}$. This can be rewritten as $^{14}$

$$
\varepsilon_{s}\left(T_{s}\right)=\left(\frac{b_{s}}{b_{s}^{*}}\right)^{-\frac{1}{\sigma}}\left(\frac{1-T_{s}}{1+\frac{T_{s}}{\varepsilon_{s}\left(T_{s}\right)}}\right)^{\frac{1}{\sigma}} .
$$

The exchange rate in the absence of a transfer in state $s$ is $\varepsilon_{s}(0)=\left(b_{s} / b_{s}^{*}\right)^{-1 / \sigma}$. It is easy to check that a small transfer to the Home country leads to a appreciation of the Home currency. ${ }^{15}$

The employment level is defined by

$$
L\left(T_{s}, W\right):=L\left(W, \varepsilon_{s}\left(T_{s}\right)\right)=\frac{\rho}{W}\left(1-T_{s}\right)
$$

When the Home country receives a transfer of $T_{s}$, denominated in the Home currency, the Foreign country transfers $T_{s}^{*}=-T_{s} / \varepsilon_{s}$ in its currency. Therefore, $L^{*}\left(T_{s}, W\right)=\rho\left(1+\left(T_{s} / \varepsilon_{s}\left(T_{s}\right)\right)\right) / W$. Transfers and labor supply are perfect substitutes. Household preferences mean that demand for the composite consumption and demand for money balance are in fixed proportion to each other. With a fixed money supply, there is no substitutability toward consumption and households consequently reduce participation in the labor market when there is a positive transfer. ${ }^{16}$

Price indices depend on the wage and the exchange rate and therefore the transfers from equations (9) and (15). Using the wage set in (5) and the labor supply (16), it can be shown that

\footnotetext{
${ }^{14}$ Note that (15) can also be derived by using (10), $T_{s}=-\varepsilon_{s} T_{s}^{*}$ and the equations for employment (see, (16) below).

${ }^{15}$ That is, $\varepsilon_{s}^{\prime}(0)=-\left(1+\varepsilon_{s}(0)\right) / \sigma<0$.

${ }^{16}$ Transfers introduce variability to labor supply. Corsetti et al. (2013) note a similar effect of transfers. However, they approximate the economy around zero transfer, so that this property plays no role in their analysis.
} 
(see Appendix A)

$$
W=\left(\kappa_{0} \frac{\mathrm{E}\left[\left(1-T_{s}\right)^{1+\psi}\right]}{\mathrm{E}\left[\left(1-T_{s}\right) A_{s}^{\gamma-1}\left(B_{s}\left(\varepsilon_{s}\left(T_{s}\right)\right)^{\gamma-1}\right]\right.}\right)^{\frac{1}{\gamma+\psi}} .
$$

Utility in state $s$ as a function of $T_{s}$ and $W$ is defined by

$$
U_{s}\left(T_{s}, W\right):=U_{s}\left(T_{s}, W, \varepsilon_{s}\left(T_{s}\right)\right)=V\left(X_{s}\left(T_{s}, W, \varepsilon_{s}\left(T_{s}\right)\right)\right)-\frac{L\left(T_{s}, W\right)^{1+\psi}}{1+\psi},
$$

where $X_{s}\left(T_{s}, W, \varepsilon_{s}\left(T_{s}\right)\right)=(\xi / \mu)\left(1 /\left(P_{s}\left(W, \varepsilon_{s}\left(T_{s}\right)\right)\right)\right)$. It can be noted that in the absence of shocks, that is, if $a_{s}=a_{s}^{*}$ is constant for all $s$, then wage and utility levels are the same under fixed and flexible exchange rates. The differences between the two regimes arises only when there is uncertainty. Thus, understanding risk sharing in each regime is key to determining which regime is better.

\subsection{Fiscal transfers, redistribution and consumption sharing}

Consumption and utility levels vary with the realization of the productivity shocks in both a currency area and under a flexible exchange rate regime. To smooth those fluctuations, countries may have the possibility to assist each other and redistribute consumption by making fiscal transfers. In a fully-fledged federation this redistribution mechanism is embedded in the fiscal system. ${ }^{17}$ In this section, we focus on transfers that equalize consumption. It is shown that, like the situation with no shocks to productivity, if there are transfers that equalize consumption, then the equilibrium allocation is the same whether there is flexible exchange rate regime or a common currency area.

Currency area In a currency area, $Y_{s}(\varepsilon)=b_{s}$ and Home consumption in state $s$ is $X_{s}\left(T_{s}, W\right)=$ $\xi\left(b_{s}+T_{s}+M_{0}\right) / P_{s}(W)$. Letting $\bar{T}_{s}$ be the transfer that equalizes the consumption levels across the two countries gives

$$
\bar{T}_{s}=\frac{1}{2}\left(b_{s}^{*}-b_{s}\right) .
$$

The transfers compensate the country with bad productivity shocks and employment levels. Anticipating these transfers, households set their wage $\bar{W}$ according to (13), so that

$$
\bar{W}=\left(\kappa_{0} \frac{\mathrm{E}\left[b_{s}^{1+\psi}\right]}{\mathrm{E}\left[b_{s} A_{s}^{\gamma-1}\right]}\right)^{\frac{1}{\gamma+\psi}} .
$$

With transfers $\bar{T}_{s}$ and the wage set to $\bar{W}$, utility in state $s$ is

$$
U_{s}\left(\bar{T}_{s}, \bar{W}\right)=V\left(\frac{\xi}{\mu} \frac{1}{P_{s}(\bar{W})}\right)-\frac{\left(L_{s}(\bar{W})\right)^{1+\psi}}{1+\psi .},
$$

where $P_{s}(\bar{W})$ and $L_{s}(\bar{W})$ are given by (11) and (12).

\footnotetext{
${ }^{17}$ Here we think of governments choosing transfers. However, since the government is benevolent in choosing for a homogenous group of households, this is equivalent to households choosing transfers to maximize long-run utility.
} 
Flexible exchange rate regime With a flexible exchange rate, local consumption is inversely related to the local price index. Equalizing consumption across the two countries, implies that price indices are equal: $P_{s}\left(W, \varepsilon_{s}\right)=P_{s}^{*}\left(W^{*}, \varepsilon_{s}\right)$. Since $P_{s}\left(W, \varepsilon_{s}\right)=\varepsilon_{s} P_{s}^{*}\left(W, \varepsilon_{s}\right)$, and because $W=W^{*}$ from the symmetry across countries, it follows that with consumption equalizing transfers, the exchange rate is fully sterilized, so that $\varepsilon_{s}=1$. Using the latter result and equation (15), we get

$$
\bar{T}_{s}=\frac{1}{2}\left(b_{s}^{*}-b_{s}\right) .
$$

These are the same transfers as under common currency. Moreover, with the transfers $\bar{T}_{s}$, it follows from (16) that $L\left(\bar{T}_{s}, W\right)=L_{s}(W)=\rho b_{s} / W$. Since employment is the same function of the wage in both the currency area and flexible exchange rate regime, the wage set in both regimes is $\bar{W}$, given by (20). Thus, when consumption is equalized across countries, welfare is the same under both flexible exchange rate and common currency regimes. We conclude that if there are differences between the two regimes, it is because of difference in risk sharing.

A utilitarian objective As an aside, we note that in the currency area, the transfers equalizing consumption, $X_{s}\left(T_{s}, W\right)=X_{s}\left(-T_{s}, W\right)$, are those that would be chosen by a utilitarian planer who maximizes the welfare objective $\mathcal{W} \equiv \mathrm{E}\left[U_{s}\left(T_{s}, W\right)+U_{s}^{*}\left(T_{s}, W\right)\right]$. This occurs because labor supply and the price indices are independent of transfers. By contrast, under a flexible exchange rate regime, the consumption equalizing transfers do not maximize this welfare objective. This is because, in the flexible exchange rate regime, transfers affect labor supply and introduce unwanted variability to the disutility of labor. Consequently, the transfers that maximize the utilitarian welfare objective tradeoff consumption equalization and employment equalization. Therefore, a utilitarian social planner in the flexible exchange rate regime would choose transfers below the consumption equalizing transfers. In what follows, we will however, concentrate on consumption equalizing transfers even in the flexible exchange rate regime. We do this for three reasons. First, because a complete analysis of the utilitarian optimum in the flexible exchange rate regime is complicated. Second, because in practice, there are likely to be political difficulties in taking labor supply variability into consideration when considering redistribution. For example, the debate about redistribution in the E.U. has not taken into account the benefits in terms of leisure. Thirdly, because our discussion below about the potential benefits of a currency area are not dependent on this distinction.

\section{Voluntary Transfers}

In this section, we consider a repeated version of the model described in Section 2 with voluntary transfers that satisfy self-enforcing or participation constraints. Countries will be prepared to make voluntary transfers when the long term benefits from future risk sharing exceed the short run costs 
of making a transfer. When transfers satisfy all such constraints, we say that they are sustainable. Whether transfers are sustainable or not will depend on parameter values.

We begin the section by comparing the welfare achieved in flexible exchange rate regime without transfers to a currency area with consumption equalizing transfers. This provides a benchmark for how well a currency area might do (absent transfers and transaction costs, the flexible exchange rate regime is always better). Next, we describe the participation constraints that need to be satisfied for transfers to be voluntary. Then, we consider for what parameter values full consumption sharing can be achieved in each regime, and for what parameter values no transfers can be sustained in each regime. We provide some analytical results when productivity shocks are small. In particular, we show that there are parameter values such that no transfers are sustained in the flexible exchange rate regime whereas full consumption sharing transfers are sustained in a currency area (the case analyzed in Section 3.1). Finally, we present numerical results on the welfare achieved in both regimes when the transfers are chosen optimally subject to the sustainability condition. This analysis shows that there is a range of plausible parameter values for which a currency area dominates.

For simplicity, from now on, we assume that there are two equiprobable states, $s=\{1,2\}$. These two states correspond to two inverse productivities $a_{G}$ and $a_{B}>a_{G}$, where by symmetry $\left(a_{1}, a_{1}^{*}\right)=\left(a_{G}, a_{B}\right)$ and $\left(a_{2}, a_{2}^{*}\right)=\left(a_{B}, a_{G}\right)$. We normalize $a_{B}=1$ and let $a_{G}=z \in(0,1)$. Thus, the Home country has the good productivity in state 1 and the bad productivity in state 2 , with the reverse being true for the Foreign country. In this two state case, we can label variables using $B$ and $G$ subscripts and avoid the asterisk notation in much of what follows. For example, we have $b_{B}=b_{G}^{*}=1 /\left((1 / 2) A^{(1-\sigma)}\right)$ and $b_{G}=b_{B}^{*}=z^{(1-\sigma)} b_{B}$ where $A^{(1-\sigma)}=\left(1+z^{(1-\sigma)}\right)$ is independent of the state. In the flexible exchange rate case, we let $\varepsilon$ be the exchange rate in state 1 and note that the exchange rate in state 2 is $1 / \varepsilon$. This two-state is widely used in the literature (see, e.g., Kehoe and Levine, 2001) and is interesting not only because it is tractable. Mundell's argument against a currency union is strongest when productivities are negatively correlated. In this example, productivities are perfectly anti-correlated and in this sense the example is designed to favor the flexible exchange rate regime. However, such a setting is also the one in which the incentives for risk sharing are the strongest. Thus, it provides a simple setting in which these opposing forces can be assessed.

\subsection{A currency area with fiscal transfers}

As a first step to examining the repeated model, this subsection compares the flexible exchange rate regime in the absence of transfers with a currency area with consumption equalizing transfers. ${ }^{18}$ That is, we compare $\mathrm{E}\left[U_{s}\left(0, W_{0}\right)\right]$, the expected utility with a flexible exchange rate regime and no transfers, with $\mathrm{E}\left[U_{s}\left(\bar{T}_{s}, \bar{W}\right)\right]$, the expected utility with the consumption sharing transfers $\bar{T}_{s}$ for

\footnotetext{
${ }^{18}$ This is the comparison considered by Ching and Devereux (2003) in a slightly different model.
} 
$s \in\{G, B\}$. That is, we ignore the issue of sustainability for the moment. Clearly, if there are no parameter values such that the currency area dominates in these circumstances, there will be no parameter values for which it ever dominates for any sustainable transfers.

Appendix B shows that the comparison between these two situations depends on only four parameters: the productivity shocks $z$, the elasticity of substitution $\sigma$, the risk aversion $\gamma$ and the inverse elasticity of labor supply $\psi$.

Proposition 1 There exists some $\hat{z}$ such that for large enough shocks $z<\hat{z}$, the currency area with consumption equalizing transfers dominates the flexible exchange rate regime with no transfers. For small enough shocks ( $z$ close to one), the currency area dominates if and only if $\gamma>\sigma(\psi \sigma-1)$. That is, for sufficiently high risk aversion, large labor supply elasticity and weak product substitutability.

\section{Proof. See Appendix B.}

The above proposition presents an important result in its own right. It shows that a currency area can be optimal, if it is associated with more redistribution of resources than would occur under a flexible exchange rate regime. To understand this, recall that consumption is variable in the flexible exchange rate regime without transfers whereas, by construction, consumption is equalized in the currency area. Employment is independent of the productivity shock in the flexible exchange rate regime without transfers because the exchange rate adjusts to productivity differences, whereas employment varies with the productivity shock in the currency area. Both the variability in employment and variability in consumption are disliked because $\psi>0$ and $\gamma>1$. Thus, a low value of $\psi$ and and a high value of $\gamma$ favor the currency area because, in that case, the cost of employment variability in the currency area is low and the cost of variability in consumption in the flexible exchange rate regime is high. The effect of a change in $\sigma$ is more complicated because changes in $\sigma$ have both level and variance effects. ${ }^{19}$ An increase in $\sigma$ means that products become more substitutable, weakening the monopoly power of firms, which leads to greater responses in production to productivity shocks. This increases the variability of employment and consumption. But, it tends to increase the variability of employment in the currency area more than the variability in consumption in the flexible exchange rate regime. Thus, a low value of $\sigma$ tends to favor the currency area. Finally, very large shocks will, without transfers, drive the consumption very low in the bad state in the flexible exchange rate regime. With constant relative risk aversion preferences, such an outcome has a very adverse consequences for expected utility. Hence, for large shocks there is a preference for a currency area.

Figure 1 shows numerical results for $z \in[0.8,1]$. It depicts the loci where currency areas with fiscal transfers yields the same expected welfare as a flexible exchange rate system without any transfers. The areas below (resp. above) those loci represents the parameters where a currency area dominates (resp. is dominated by) a flexible exchange rate system. Currency areas are preferred for stronger risk

\footnotetext{
${ }^{19}$ Changes in $\gamma$ and $\psi$ have indirect level effects through changes in the wage.
} 
aversion (higher $\gamma$ ), more elastic labor supply (lower $\psi$ ) and lower elasticity of substitution (lower $\sigma$ ), in line with Proposition 1. In addition, the figure shows that currency areas are preferred for larger productivity shocks (lower $z$ ). Larger productivity shocks increase the variability of consumption and hence, the desire for more risk sharing. Typical estimates from the literature put the value of relative risk aversion $\gamma$ are in a range between 2 and 7, labor supply elasticity $\psi^{-1}$ in an interval encompassing values slightly below $1^{20}$ and elasticity of product substitution in a range between 2 and $8 .^{21}$ The preference for common currency area with fiscal transfers depends on the actual values of these parameters. Finally, the figure also suggests that shocks need to be quite large for currency areas to dominate for all parameter values. However, it is likely to dominate for sufficiently high elasticity of labor supply and low elasticities of product substitution.

The above discussion leaves unexplained the reason why a currency has risk-sharing transfers and the flexible exchange rate regime has none. ${ }^{22}$ However, the comparison may be relevant in some circumstances. It is certainly conceivable that institutional factors play a role in enforcing fiscal transfers within a currency area. For instance, where a currency area coincides with a legal state, the constitution may provide a legal framework for redistributive policies. However, it is less clear how the redistributive policies may be implemented in an association of sovereign states that nevertheless belong to a common currency area. In the next sub-sections, we examine how the adoption of common currency may enhance risk sharing mechanisms even absent a legal framework for redistribution.

\subsection{Participation constraints}

In this section we describe a repeated version of the model considered in Section 2 and the participation constraints that transfers must satisfy for them to be voluntary.

To discuss the possibility of voluntary transfers, we assume that countries interact repeatedly over an infinite horizon and let all households and countries have the same discount factor $\delta \in(0,1)$.

\footnotetext{
${ }^{20}$ The impact of labor supply elasticity on the preference for a currency area is discussed by Devereux (2004) in a static model with preference shocks.

${ }^{21}$ Todter (2008) assesses the coefficients of relative risk aversion in the range $\gamma \in[1.4,7.1]$. Backus et al. (1992) and Corsetti et al. (2008) use a low value $\gamma=2$. Basu and Fernald (1997) estimate of elasticity of substitution in a range $\sigma \in[4,6]$. Mendoza (1991) using a sample of industrialized countries sets that elasticity equal to $\sigma=3.8$. Stockman and Tesar (1995) estimate a lower elasticity $\sigma=1.8$. It is generally agreed that $\sigma$ is low for traded products. For example, Corsetti et al. (2008) estimates that the short term volatility of real exchange rates is consistent with $\sigma$ slightly lower than one for traded goods. However, because our model does not distinguish the elasticities of traded and local goods and because there is monopolistic competition, it is compatible only with $\sigma>1$. Thus, in the baseline numerical computations presented later, we use $\sigma=2$. Estimates of the Frisch elasticity range from 0.1 to more than 1.0, but cluster around 0.4. Devereux (2004) explores the labor supply elasticity in the range $\psi^{-1} \in[0.6,2.2]$. Reichling and Whalen (2015) suggest the range [0.27, 0.53] for $\psi^{-1}$.

${ }^{22}$ Alesina et al. (1995) and Persson and Tabellini (1996) that a currency area is preferred because of public goods or externalities.
} 


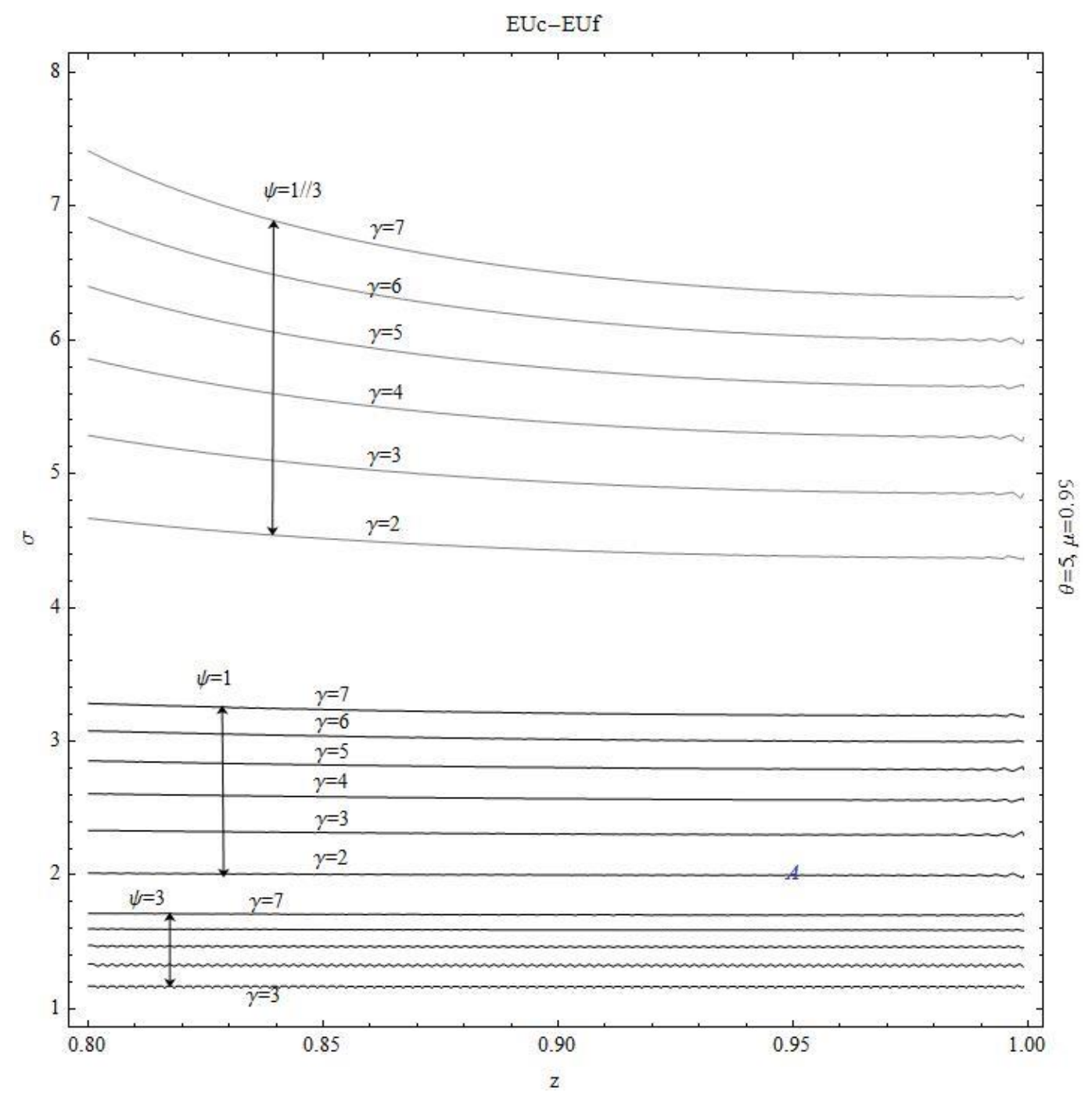

Figure 1: LOCI OF INDIFFERENCE BETWEEN A COMMON CURRENCY WITH CONSUMPTION SHARING TRANSFERS AND A FLEXIBLE EXCHANGE RATE REGIME WITH NO TRANSFERS FOR A RANGE OF VAlUeS OF $\psi$ AND $\gamma$. OTher PARAMETER VAlues: $\theta=5$ AND $\mu=$ 0.99. The CURRENCY Union DOMinates FOR LARGER SHOCKS (LOWER $z$ ) AND FOR LESS COMPETITIVE ENVIRONMENTS (LOWER $\sigma$ ).

For tractability of the analysis, we assume that there exists no asset that can be transferred between time periods. As claimed in the introduction, we take the view that money and asset holdings by households are not sufficiently large to permit any consumption smoothing. ${ }^{23}$ Since transfers cannot be legally enforced, countries can renege on any agreement if they find it in their interest not to make a transfer. Hence any transfer programme has to be designed to be self-enforcing. We apply an

\footnotetext{
${ }^{23}$ In this paper, money holdings are deemed to be ineffective in transmitting wealth from one period to the other. This assumption is made to highlight our analysis of the incentives for risk sharing through voluntary transfers mechanisms.
} 
approach similar to Thomas and Worrall (1988) who examine self-enforcing wage contracts between employers and employees. Thus, we presume that countries make a tacit agreement that specifies a state contingent transfer to be made from the good to the bad productivity country. ${ }^{24}$

For the sake of conciseness, let $\mathcal{T}$ denote the transfer received by the country with the bad shock (Foreign in state 1 and Home in state 2) and $\epsilon$ be the exchange rate when Home has the good shock (in state 1 ). That is, $\varepsilon_{G}=: \epsilon$, and hence, $\varepsilon_{B}=1 / \epsilon$. Also, $T_{B}=: \mathcal{T}$ and $T_{G}=-\epsilon \mathcal{T}^{25}$ Such a formulation allows us to dispense with the state $s$ subscript on transfers and exchange rates without introducing confusion. Since the wage is set before transfers, and transfers are summarized by $\mathcal{T}$, the equilibrium wage is a function of $\mathcal{T}$, which we write as $W(\mathcal{T})$. In the currency area, $\epsilon=1$, whereas in the flexible case, the exchange rate is also a function of $\mathcal{T}$, defined by applying $T_{G}=-\epsilon \mathcal{T}$ to equation (15),

$$
\epsilon(\mathcal{T})=\left(\frac{b_{G}}{b_{B}}\right)^{-\frac{1}{\sigma}}\left(\frac{1+\epsilon(\mathcal{T}) \mathcal{T}}{1-\mathcal{T}}\right)^{\frac{1}{\sigma}} .
$$

As stated above, we assume that any breach of this tacit agreement results in a breakdown in which no transfers are made. Let $\Delta U_{G}(\mathcal{T}):=U_{G}(-\epsilon(\mathcal{T}) \mathcal{T}, W(\mathcal{T}))-U_{G}(0, W(0))$ denote the net change in utility from the addition of a transfer $\mathcal{T}$, where utility is defined in equations (14) or (18) depending on the exchange rate regime. The term $\Delta U_{G}(\mathcal{T})$ is the short term loss in utility from making the prescribed transfer in the good state relative to no transfer being in place. Let $\Delta U_{B}(\mathcal{T})$ be defined similarly as the short term gain in utility from being in receipt of the transfer. The expected net gain of a country in the good state of making the transfer over not making the transfer is given by

$$
V_{G}(\mathcal{T}):=U_{G}(-\epsilon(\mathcal{T}) \mathcal{T}, W(\mathcal{T}))-U_{G}(0, W(\mathcal{T}))+\frac{\delta}{(1-\delta)}\left(\frac{1}{2} \Delta U_{G}(\mathcal{T})+\frac{1}{2} \Delta U_{B}(\mathcal{T})\right)
$$

The first term is the initial short term loss of making the transfer given that the wage will not adjust in the short term but is set beforehand as if the transfer were to be made. The second term is the long term expected benefit from being in the transfer scheme relative to the situation with no transfers. This equation can be rewritten as

$$
V_{G}(\mathcal{T})=U_{G}\left(0, W_{0}\right)-U_{G}(0, W(\mathcal{T}))+\frac{1}{(1-\delta)}\left(\left(1-\frac{\delta}{2}\right) \Delta U_{G}(\mathcal{T})+\left(\frac{\delta}{2}\right) \Delta U_{B}(\mathcal{T})\right)
$$

where $W_{0}:=W(0)$. A similar expression holds for the expected net gain of a country in the bad state in receipt of a transfer, $V_{B}(\mathcal{T})$. The transfer $\mathcal{T}$ is sustainable if and only

$$
V_{G}(\mathcal{T}) \geq 0
$$

It is clear that if $V_{G}(\mathcal{T}) \geq 0$, then $V_{B}(\mathcal{T}) \geq 0$, so that provided the inequality (23) is satisfied, the transfer $\mathcal{T}$ is sustainable. Clearly $V_{G}(0)=0$.

\footnotetext{
${ }^{24}$ We consider a stationary state in which transfers are not history dependent. With two states of nature, convergence to the stationary state occurs as soon as both states have been realized.

${ }^{25}$ By symmetry $T_{G}^{*}=T_{B}$ and $T_{B}^{*}=T_{G}$.
} 


\subsection{Sustaining consumption sharing}

In this subsection, we study the set of discount factors for which countries will voluntarily participate in consumption equalizing transfers.

The consumption equalizing transfers are: $\mathcal{T}=\overline{\mathcal{T}}=(1 / 2)\left(b_{G}-b_{B}\right)=\left(z^{1-\sigma}-1\right) /\left(1+z^{1-\sigma}\right) .{ }^{26}$ With these transfers, households set their wages to $\bar{W}:=W(\overline{\mathcal{T}})$. The net gain of making the transfer in the good state is given by equation (22) and this system of transfers is sustainable if and only if inequality (23) is satisfied for transfer $\overline{\mathcal{T}}$ :

$$
V_{G}(\overline{\mathcal{T}})=U_{G}\left(0, W_{0}\right)-U_{G}(0, \bar{W})+\frac{1}{(1-\delta)}\left(\left(1-\frac{\delta}{2}\right) \Delta U_{G}(\overline{\mathcal{T}})+\left(\frac{\delta}{2}\right) \Delta U_{B}(\overline{\mathcal{T}})\right) \geq 0
$$

where $W_{0}$ is the equilibrium wage without transfers. Provided $\Delta U_{G}(\overline{\mathcal{T}})+\Delta U_{B}(\overline{\mathcal{T}})>0$, a larger discount factors relaxes this inequality. ${ }^{27}$ In this case, we may compute a critical discount factor $\bar{\delta}$ above which consumption equalizing transfers are sustainable. The analytical value of this discount factor for each exchange rate regime is provided in Appendix C. For the sake of conciseness, we concentrate here on the case where shocks are small (we linearize about $z=1$ ) and for the particular parameter values $\mu=\psi=(\theta-1) / \theta=1$ (that is, for small money holdings, intermediate labor supply elasticity and zero market power for workers). ${ }^{28}$ This allows us to concentrate on the impact of small productivity shocks $z$, risk aversion $\gamma$, and elasticity of substitution $\sigma$. In this case, the critical discount factor $\bar{\delta}$ is determined by:

$$
\begin{aligned}
& \bar{\delta}^{c} \approx 1+\frac{1}{4}\left(\frac{\gamma((1+\sigma)+\gamma(\sigma-1))}{1+\gamma}\right)\left(\frac{\sigma-1}{\sigma}\right)(z-1), \\
& \bar{\delta}^{f} \approx 1+\frac{1}{4}\left(\frac{(\gamma-\sigma(\sigma-1))((1+\sigma)+\gamma(\sigma-1))}{(1+\gamma) \sigma^{2}}\right)\left(\frac{\sigma-1}{\sigma}\right)(z-1),
\end{aligned}
$$

where the superscripts $c$ and $f$ refer to the currency area and flexible regime cases.

The above approximations illustrates three important points. First, the RHS of (25) is less than one and the critical discount factor $\bar{\delta}^{c}$ falls with the size of the productivity shock (as $z$ decreases). Second, the expression on the RHS of (26) is also less than one and falls with the size of the productivity shock provided $\gamma>\sigma(\sigma-1)$. However, when $\gamma \leq \sigma(\sigma-1)$, the critical discount factor is equal to one and it is not possible to sustain consumption equalizing transfers in the flexible

\footnotetext{
${ }^{26}$ Recall that with the consumption equalizing transfer, $\epsilon(\overline{\mathcal{T}})=1$ in the flexible exchange rate regime.

${ }^{27}$ This is true in the flexible regime case and true in the common currency case for large enough $\mu$. See Appendix C for details.

${ }^{28}$ Although we choose these parameter values to simplify the presentation, there are also sound reasons underlying these choices. A value of $\psi=1$ is within the normal range of empirical estimates. Choosing $\mu=1$ reflects our focus on situations where money holdings are comparatively small. Finally, numerical simulations show that results are not very sensitive to the choice of $\theta$. Equally, it is to be expected that the demand for insurance in higher when shocks are larger and that this would give an advantage to currency areas if transfers can be sustained. By focusing on small shocks, we show that currency areas may, for some parameter values, dominate.
} 
exchange rate regime. ${ }^{29}$ Thirdly, it is easily seen from the above equations that $\bar{\delta}^{c}<\bar{\delta}^{f}$ because $\gamma>(\gamma-\sigma(\sigma-1)) / \sigma^{2}$. Hence, there are parameter configurations for small shocks where consumption equalizing transfers are sustained in a currency area but where they cannot be sustained under a flexible exchange rate system (we present numerical results below to show the same is also true for larger shocks). All these results hold for more general parameterizations provided $\mu$ is close to one and $\sigma>\psi^{-1}$. These observations are summarized by:

Proposition 2 Assuming that productivity shocks are small (z close to 1), consumption equalizing transfers are more likely to be sustained for larger shocks in a currency area. This is true in the flexible exchange rate system for $\gamma>\sigma(\sigma \psi-1)$. If $\mu$ is close to 1 and $\sigma>\psi^{-1}$, consumption equalizing transfers are more likely to be sustained under currency areas than under flexible exchange rate systems $\left(\bar{\delta}^{c}<\bar{\delta}^{f}\right)$.

Proof. See Appendix C for the detailed derivation of the above formulas.

It is also possible to see from equation (25) that the approximation $\bar{\delta}^{c}$ is decreasing with $\gamma$ and $\sigma$. That is, greater risk aversion and stronger product substitutability increase the incentives to sustain the transfers in the currency area. These properties are intuitive. Greater risk aversion means households have a stronger preference for equalizing consumption and are less willing to substitute their consumption by changing their labor supply. Incentives are also stronger when products are better substitutes. Higher product substitutability intensifies competition between firms leading to larger responses in production to a productivity shock. This raises the variance in income and consumption, increasing the preference of households to smooth their consumption with transfers. The same comparative statics for $\bar{\delta}^{f}$ are not as trivial because of the exchange rate effect.

Although analytical results for large shocks are difficult to obtain, the exact values of the critical discount factors can be easily computed (see Appendix C). Figure 2 shows the critical discount factors for large shocks and a particular parametrization (shown in the figure). The solid lines plot $\bar{\delta}^{c}$ (thick) and $\bar{\delta}^{f}$ (thin) as a function of $z$ (ignore the dashed lines for the moment). The figure confirms that the consumption sharing transfers are sustained for lower discount factors under a currency area $\left(\bar{\delta}^{c}<\bar{\delta}^{f}\right)$. These properties can be reproduced for other relevant parameter values, such as those in the ranges discussed in the section $3.1 .^{30}$

\footnotetext{
${ }^{29}$ The reason is that under the flexible exchange rate regime, the transfers that equalize consumption, do not maximize expected utility and therefore may be rejected even by countries that are complete patient $(\delta=1)$.

${ }^{30}$ Although the critical discount factor $\bar{\delta}^{f}<1$ for all shocks shown in Figure 2, it can also be confirmed that $\bar{\delta}^{f}=1$ for a range of shocks for different parameter values. For example, for the parameters $\sigma=4, \gamma=5, \psi=1, \theta=5$ and $\mu=0.99$, it can be computed that $\bar{\delta}^{f}=1$ for $z \in(0.53375,1]$. Thus, for these parameter values, consumption sharing can never be sustained under a flexible exchange rate regime even for some larger shocks.
} 


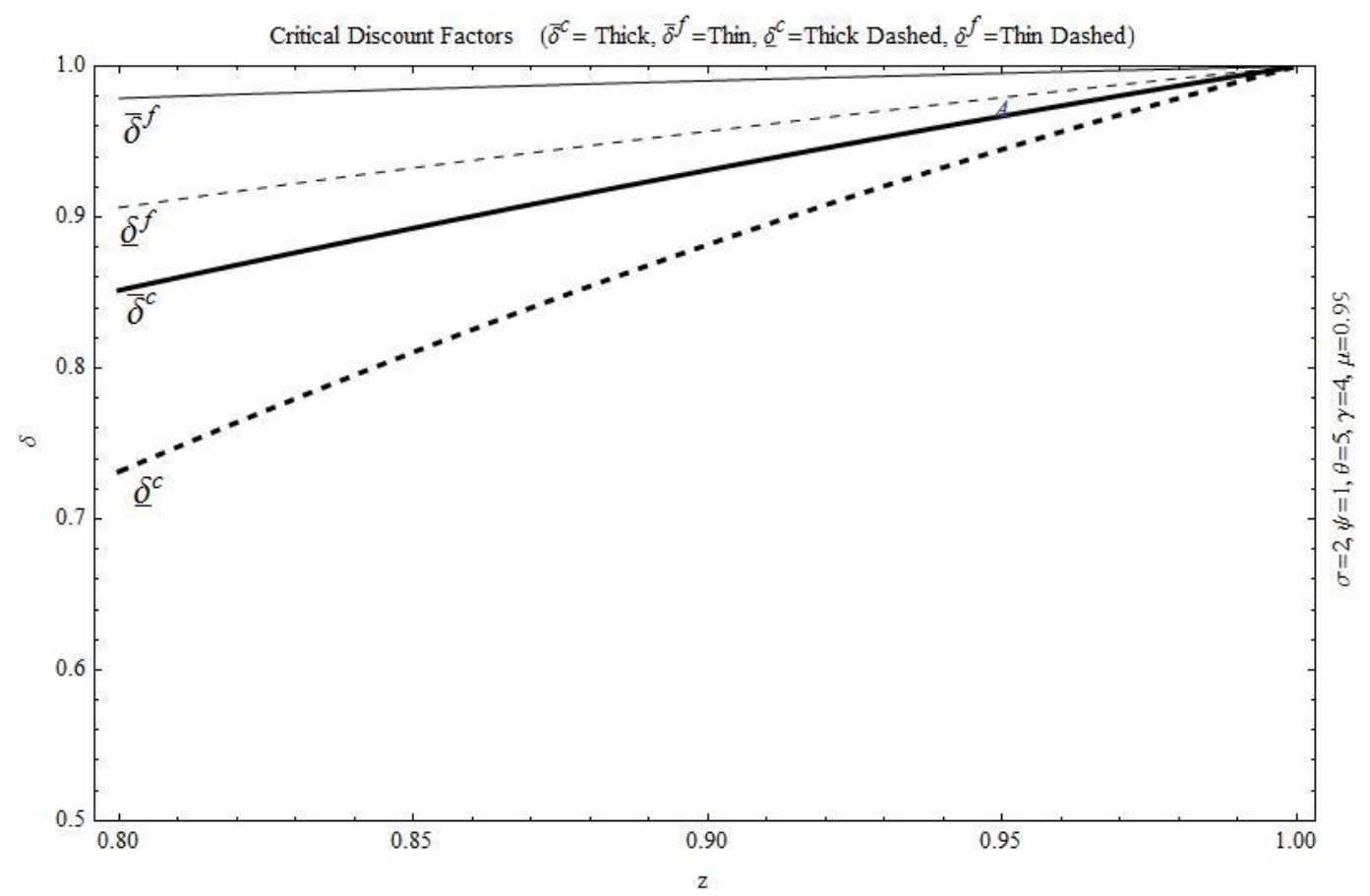

Figure 2: Critical discount factors as a function of the Shock $z$. The Solid THICK LINE CORRESPONDS TO $\bar{\delta}^{c}$ AND THE SOLID THIN LINE TO $\bar{\delta}^{f}$. THE DASHED THICK Line CORRESPonds to $\underline{\delta}^{c}$ (SEE SECTION 3.4) ANd the DAShed thin Line to $\underline{\delta}^{f}$. PARAMETER VALUES: $\sigma=2, \gamma=5, \psi=1, \theta=5$ AND $\mu=0.99$.

\subsection{Sustaining transfers}

We now consider the case where it is not possible for the countries to sustain any transfers. This will occur when the discount factor is low. In the same way the previous section considered critical discount factors above which the consumption equalizing transfers could be sustained, this section will consider the critical discount factors below which no small transfers can be sustained. We have $V_{G}(0)=0$. If $V_{G}^{\prime}(0)>0$, then a small increase in transfer above zero is sustainable; if $V_{G}^{\prime}(0) \leq 0$, then no marginally small transfers will be sustainable. Small transfers create several effects. A transfer reduces the purchasing power of the giving country causing its households to increase their labor supply. The opposite effect takes place in the recipient country. In addition, small transfers affect wage setting and the exchange rate in the flexible exchange rate case. The overall impact of these effects on the sign of $V_{G}^{\prime}(0)$ is complex and examined in Appendix D. Again, for conciseness, we focus on the case where shocks are small ( $z$ close to one) and $\mu=\psi=(\theta-1) / \theta=1$ (the general case being treated in Appendix D). For small shocks, it is shown in Appendix D that the critical discount factor $\underline{\delta}$ is approximated by

$$
\underline{\delta}^{c} \approx 1+\frac{1}{2}\left(\frac{\gamma(\sigma+\gamma(\sigma-1))}{1+\gamma}\right)\left(\frac{\sigma-1}{\sigma}\right)(z-1),
$$


in the case of the currency area and by

$$
\underline{\delta}^{f} \approx 1+\frac{1}{2}\left(\frac{\gamma(\sigma+\gamma(\sigma-1))+\gamma(\sigma-1)^{2}+\sigma(1+\sigma)}{(1+\gamma) \sigma^{2}}\right)\left(\frac{\sigma-1}{\sigma}\right)(z-1),
$$

in the flexible exchange rate case. It is easily seen that the expressions in the large brackets of (27) and (28) are positive, so that $\underline{\delta}^{c}$ and $\underline{\delta}^{f}$ are less than one and fall with smaller $z$. Equally, from equations (27) and (28), it is easily checked that a sufficient condition for $\underline{\delta}^{c}<\underline{\delta}^{f}$ is $2 \sigma^{2}-3 \sigma-1 \geq 0$, or $\sigma \geq(3+\sqrt{17}) / 4 \approx 1.7808 .^{31}$

By comparing equations (25) and (27), and equations (26) and (28), it can also be seen that for small shocks $\underline{\delta}^{c}<\bar{\delta}^{c}$ and $\underline{\delta}^{f}<\bar{\delta}^{f}$. In summary, it can be shown that:

Proposition 3 For small productivity shocks, transfers are more likely to be sustained for larger shocks (smaller $z)$ in a currency area. The same holds in the flexible exchange rate regime for $\mu, \phi$ and $(\theta-1) / \theta$ close to one. For $\sigma>\psi^{-1}$ and $\mu$ close to one, $\bar{\delta}^{c}>\underline{\delta}^{c}$. For $\mu, \phi$ and $(\theta-1) / \theta$ close to one, $\bar{\delta}^{f}>\underline{\delta}^{f}$. A sufficient condition for $\underline{\delta}^{c}<\underline{\delta}^{f}$ is $\sigma>1+(\theta /(\theta-1))$.

Proof. See details in Appendix D.

Returning to the the case of larger shocks, Figure 2 depicts critical discount factor for various shocks in the case of currency area and flexible exchange rates; $\underline{\delta}^{c}$ (thick dashed line) and $\underline{\delta}^{f}$ (thin dashed line) for the parameter values $\mu=0.99, \psi=1, \theta=5, \sigma=2$ and $\gamma=5$. In this numerical example, transfers are sustained for lower discount factors under currency area because $\underline{\delta}^{c}$ is smaller than $\underline{\delta}^{f}$. Again, similar properties can be shown to hold for for other relevant parameter values.

Looking at Figure 2, it can be seen that $\underline{\delta}^{c}<\bar{\delta}^{c}<\underline{\delta}^{f}<\bar{\delta}^{f}<1$. This shows that transfers are more likely to be sustained under currency areas. Consider a fixed shock $z$, and an increases in households' impatience (lowering $\delta$ ). First, for low impatience, countries can sustain consumption equalizing transfers in both regimes. As impatience increases, countries become unable to sustain consumption equalizing transfers under a flexible exchange rate regime. Then, as impatience increases further, they become unable to sustain any transfers in this regime. For higher levels of impatience, consumption equalizing transfers become unsustainable in a currency area and as households become even more impatient, no transfers can be sustained even in the currency area case. Within a currency area, the cost of having no transfers is higher because adjustments in the presence of the wage rigidity cannot be made through the exchange rate flexibility. This cost increases the incentive to sustain more transfers. As we will show next, this effect can improve welfare in the currency area relative to the flexible exchange rate regime.

\footnotetext{
${ }^{31}$ This condition is valid for $\gamma=1$. It is easily checked that the condition on the value of $\sigma$ above which $\underline{\delta}^{c}<\underline{\delta}^{f}$ falls as $\gamma$ increases. Thus, the above condition is sufficient for any $\gamma>1$. For a higher $\gamma$, the corresponding required value of $\sigma$ is lower.
} 


\subsubsection{Sustainable fiscal transfers}

As we have seen voluntary transfers are a substitute for exchange rate flexibility because they allow greater consumption smoothing. We turn now to whether these risk sharing benefits offset the costs of a fixed exchange rate. In this section, we consider the possibility that (i) consumption equalizing transfers are sustained in a currency area regime and no transfers are sustained in the flexible exchange rate regime and (ii) the former regime yields a higher expected welfare than the latter. In Section 3.1, we considered the case with consumption sharing transfers in a currency area regime and no transfers in the flexible exchange rate regime but without considering sustainability. Here we address the possibility that this condition is the optimal outcome given the participation constraints (23). Condition (i) holds for any discount factors $\delta \in\left[\bar{\delta}^{c}, \underline{\delta}^{f}\right]$ where $\underline{\delta}^{f}>\bar{\delta}^{c}$. Condition (ii) is satisfied if $\mathrm{E}_{s} U_{s}^{c}\left(\bar{T}_{s}, \bar{W}\right) \geq \mathrm{E}_{s} U_{s}^{f}\left(0, W_{0}^{f}\right)$ where $W_{0}^{f}$ is the wage set in the flexible exchange rate system. Such a case is illustrated by the point A at $(z, \delta)=(0.95,0.97)$ in Figures 1 and 2. For those parameters, Figure 1 shows that a currency area with such transfers yields a higher expected welfare than a flexible exchange rate without transfers while Figure 2 shows that consumption sharing transfers are sustained in a currency area regime and no transfers are sustained under flexible exchange rates.

For small shocks, condition (i) is determined by comparing (25) and (28). Appendix B shows that the condition (ii) holds for high enough risk aversion, $\gamma>\sigma(\psi \sigma-1)$. Focusing again on the parameters $\mu=\psi=(\theta-1) / \theta=1$ for the sake of clarity, we can state that there exists a non-empty set of parameters such that conditions (i) and (ii) are satisfied for small shocks if and only if

$$
\begin{aligned}
(\sigma-1)\left(\sigma^{2}-2\right) \gamma^{2}+(\sigma-1)\left(\sigma^{2}+2\right) \gamma-2 \sigma(\sigma+1) & >0 \\
\gamma-\sigma(\sigma-1) & >0
\end{aligned}
$$

Many parameters satisfy these conditions. For example, a sufficient condition for (29) to be satisfied is $\sigma>1+\sqrt{2}$. Of course, a higher $\sigma$ means $\gamma$ must be higher to satisfy (30). ${ }^{32}$ For instance, the parameters of Figures 1 and $2(\sigma=2, \gamma=4)$ imply that conditions (i) and (ii) are satisfied if $\delta$ lies between $\underline{\delta}^{f} \approx 1+0.42(z-1)$ and $\bar{\delta}^{c} \approx 1+0.70(z-1)$. We summarize this discussion with the following proposition.

Proposition 4 For small productivity shocks, there exist discount factors such that currency areas are preferred to flexible exchange rate systems under condition (29) and (30). In this case, consumption sharing transfers are sustained in the currency area whereas no transfers are sustained under flexible exchange rates. In particular, for $\mu$ close to one and $\theta$ large, a sufficient condition for $\underline{\delta}^{f}>\bar{\delta}^{c}$ is $\sigma>1+\sqrt{(1+3 \psi) /(1+\psi)}$.

Proposition 4 presents the conditions for the optimality of currency areas when they implement a transfer that sustains consumption sharing. This result qualifies Mundell's argument about currency

\footnotetext{
${ }^{32}$ Both conditions (30) and (29) are satisfied for $\sigma=2$ and $\gamma \geq 2$.
} 
unions. Mundell argues that a currency cannot be optimal if shocks are not positively correlated. The above proposition shows that negatively correlated shocks harm households when there are no transfers between countries. However, wage stickiness increases the consumption variance and increases risk sharing incentives in a currency area. For a set of parameter values, the consumption sharing transfers become sustainable and a currency area may outperform the flexible exchange rate regime. That is, a currency union may be optimal without the need for any form of contract between countries, such as fiscal treaties, common constitution, etc.

We next consider whether currency areas can be welfare-improving even when the only sustainable voluntary transfers are below the full consumption sharing transfers.

\subsection{Constrained transfers}

In this section, we discuss steady-state welfare when transfers are chosen to maximize welfare subject to the participation constraints. The consumption equalizing transfer $\overline{\mathcal{T}}$ is chosen if it is sustainable $(\delta>\bar{\delta})$ and a zero transfer is chosen if no transfer is sustainable $(\delta<\underline{\delta})$. Otherwise, welfare is maximized by choosing a transfer $\hat{\mathcal{T}} \in(0, \overline{\mathcal{T}})$ such that the participation constraints is binding in the good state of nature; that is, when $V_{G}(\hat{\mathcal{T}})=0$. Because analytical results are difficult to obtain, we present simulations that indicate the parameter values for which currency areas dominate or not.

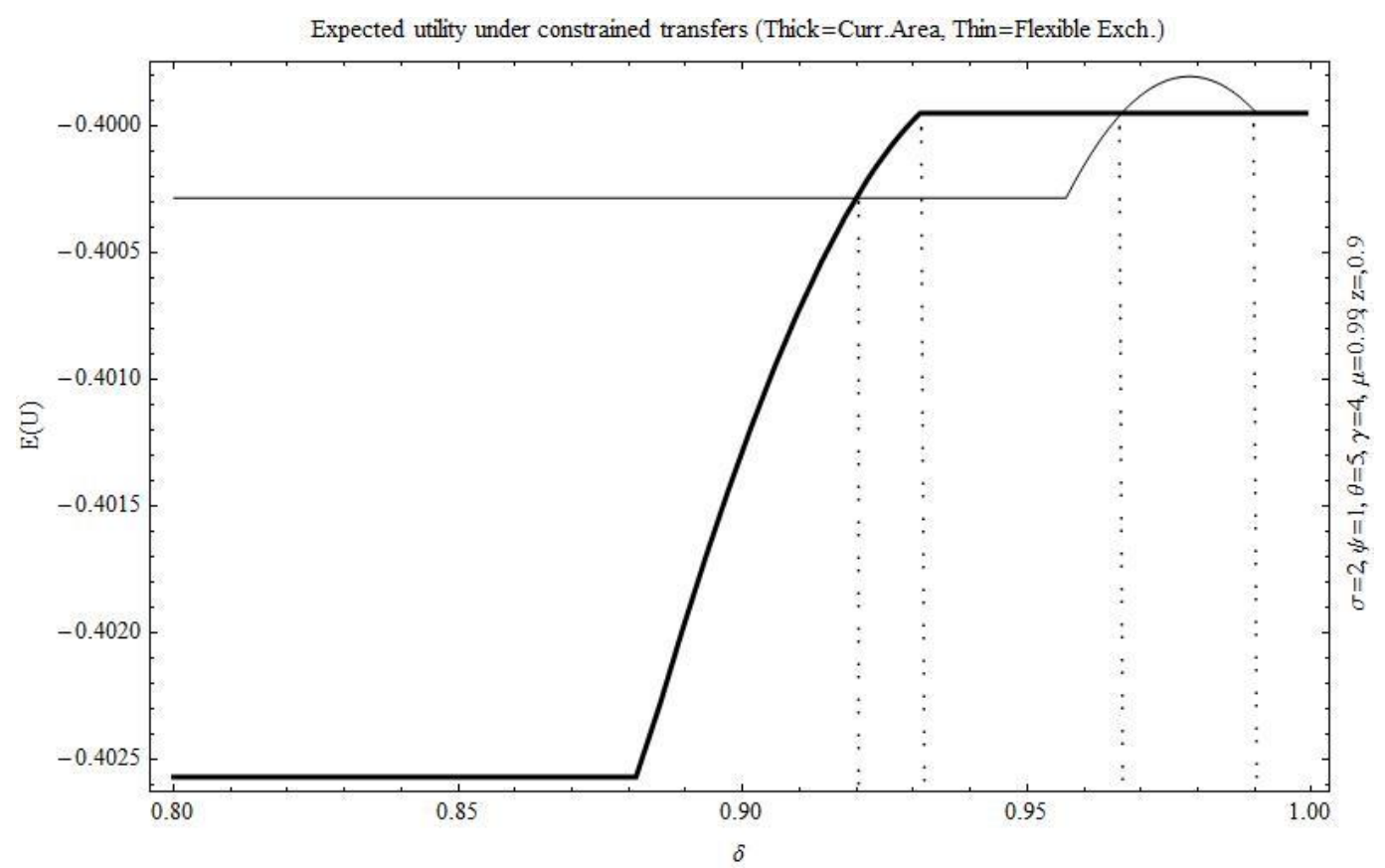

Figure 3: Steady State Expected Utility with Constrained Transfers as a Function of the Discount Factor. Thick line - Currency Area; Thin line Flexible exchange Rate Regime. Parameter values: $\sigma=2, \gamma=5, \psi=1, \theta=5$, $\mu=0.99$ AND $z=0.9$. 


\begin{tabular}{cccccccc}
\hline \hline & $\delta$ & $z$ & $\gamma$ & $\psi$ & $\sigma$ & $\theta$ & $\mu$ \\
\hline \hline Basis & 0.950 & 0.900 & 5.00 & 1.00 & 2.00 & 5.00 & 0.99 \\
\hline \hline Min & 0.902 & 0.860 & 2.40 & 0.00 & 1.40 & 1.20 & 0.40 \\
$\operatorname{Max}$ & 0.965 & 0.946 & 11.80 & 1.58 & 2.40 & $\infty$ & 0.99 \\
\hline \hline
\end{tabular}

Table 1: Sensitivity Analysis. The table reports the minimum and maximum of the range of each parameter for which currency areas dominate. It centers the ranges about the parameters $(\delta, z, \gamma, \psi, \sigma, \theta, \mu)=(0.95,0.90,5,1,2,5,0.99)$.

Figure 3 shows the steady-state expected utility under both exchange rate regimes for different values of the discount factor and for a given set of parameter values. For low values of the discount factor $\delta<0.870$, no transfers are sustainable in either regime and the flexible exchange rate regime dominates as exchange rate absorbs the shocks. For $\delta \in[0.870,0.921]$, the currency area sustains constrained transfers while the flexible exchange rate regime supports no transfers at all. As $\delta$ rises, the constrained transfers increase and improve risk-sharing and welfare in the currency area. But this is not sufficient to outweigh the absence of an exchange rate mechanism. For $\delta \in(0.920,0.932]$, the constrained transfers raise the welfare in the currency area above that under flexible exchange rates. A currency area sustains greater risk-sharing through transfers, but also provides a harder landing in the event of a default on transfers. For $\delta \in(0.932,0.966]$, full consumption sharing is sustained and the currency area generates greater welfare. At approximately $\delta=0.966$, transfers are sustained in the flexible exchange rate regime and both regimes generate the same welfare. For $\delta>0.966$, flexible exchange rates dominate. ${ }^{33}$ Finally, for high enough discount factors, $\delta>0.990$, the consumption-equalizing transfer can be sustained in either regime, so that both regimes yield the same welfare.

As discussed above, currency areas sustain transfers and are optimal for a range of discount factors. It is instructive to study the robustness of this result to changes in other parameters. Table 1 present a sensitivity analysis around the parameters presented in the previous figures. More precisely, we set the parameters to the basis values $(\delta, z, \gamma, \psi, \sigma, \theta, \mu)=(0.95,0.90,5,1,2,5,0.99)$, for which a currency area with voluntary transfers is optimal. For each parameter, we then compute the range within which a currency area dominates keeping the other parameters unchanged. Table 1 reports the minimum and maximum value of a parameter for which currency areas dominate.

To interpret this table, remember that with no transfers, both consumption and employment are variable in a currency area, whereas employment is insulated from shocks in the flexible exchange rate regime. For large $\mu$, consumption variability is larger in the currency area than in flexible exchange rate regime. Thus, with no transfers, the flexible exchange rate regime is preferred. Equally, if in

\footnotetext{
${ }^{33}$ The hump shape in welfare under flexible exchange rates, arises because we consider transfers up to the consumption equalizing transfers, which, as discussed above, do not maximize welfare in this regime.
} 
both regimes there are transfers that equalize consumption, then the flexible exchange rate regime is again preferred. A currency area can only dominate if it sustains greater transfers than the flexible exchange rate regime.

A currency area can sustain more transfers for some parameter values. To understand this, recall that consumption variability, and employment variability in the currency area, decrease in $z$ (shocks are larger for smaller $z$ ) and increase in $\sigma$. The latter because, as discussed in Section 3.1, higher product substitution (higher $\sigma$ ) decreases firms' product market power and raises their incentives to respond to shocks with changes in production and employment. An increase in $\gamma$, increases the demand for insurance, whereas an increase in $\psi$ raises the cost of variable employment. ${ }^{34}$ The cost of variability of employment is unaffected by transfers in the currency area except to the extent that there are indirect effects through changes in the wage. There are similar effects in the flexible exchange rate regime, except that consumption variability is affected only indirectly by transfers through the wage and exchange rate and transfers and employment are substitutes.

Considering Table 1, it can be seen first, that the threshold values for the discount factors are consistent to our earlier discussion. Second, for small shocks, no transfers are sustained in either regime and a flexible exchange rate system is optimal. Equally for larger shocks, consumption is equalized in both regimes and the flexible exchange rate regime again dominates. However, for intermediate values, $z \in(0.856,0.935)$, the currency area sustains more transfers and dominates the flexible exchange rate regime. Third, greater risk aversion increases the demand for transfers and makes the costs of a currency area without transfers higher. Therefore, for a range of $\gamma$, higher transfers can be sustained in the currency area than in the flexible exchange rate regime. ${ }^{35}$ This in turn means the currency area dominates in terms of welfare for some range of $\gamma$. Fourth, for a high value of $\psi$, the costs of variability of employment in a currency area are high and a currency area, whereas for low $\psi$, such costs are small. ${ }^{36}$ Fifth, a higher $\sigma$ increases variability and increases the demand for insurance. As with higher risk aversion, the currency area dominates for intermediate values of $\sigma$. Finally, a currency area dominates for a wide range of $\mu$ and $\theta$. In particular, our simulations show that welfare is fairly insensitive to changes in the preference for money holdings and the elasticity of substitution between labor services.

The parameter ranges given in Table 1 are quite plausible. A $4 \%$ interest rate corresponds to a discount fact of $\delta=0.962$, just within the range given in Table 1 . A value of $z=0.935$ corresponds to a standard deviation of the productivity shock of $3.25 \%$. A coefficient of risk aversion greater than $\gamma=2.4$ is in the range of typical empirical estimates found in the literature (see, e.g., Todter, 2008), though it might also be considered quite high. Any labor supply elasticity $\psi^{-1}>0.66$ is

\footnotetext{
${ }^{34}$ There are indirect effects of changes in parameter values that occur through changes in the wage. These indirect effects are incorporated in the computations, but the indirect effects on utility are quite small.

${ }^{35}$ Exchange rate fluctuations only depend indirectly on risk aversion through the effect of risk aversion on transfers.

${ }^{36}$ There are indirect effects of changes in $\psi$ on wage setting. A higher value of $\psi$ typically leads to lower wages and prices and higher consumption.
} 
within or close to the range studied in the literature (see, e.g., Devereux, 2004). Similarly, the values for $\sigma$ presented in Table 1 are close to the ones proposed in the literature (see, e.g., Mendoza, 1991; Stockman and Tesar, 1995). Our claim here is not to have demonstrated the optimality of currency areas in all situations. We have shown that it can be beneficial for plausible parameter values in a simple example. However, we do want to conclude from our discussion that the potential for different risk sharing possibilities is an important determinant of optimum currency areas, that should not be left out of account.

\section{Transaction costs}

So far, our analysis has been weighted against the currency area except to the extent that it sustains greater fiscal transfers. However, one advantage of currency areas lies in the elimination of transaction costs in exchanging currencies (see, e.g., Alesina and Barro, 2002; Bayoumi, 1994). In this section, we check the robustness of the previous analysis by adding iceberg transaction costs of the type considered by Samuelson (1954). In particular, to receive one unit of the foreign currency, domestic consumers must purchase $\tau>1$ units of foreign currency. They therefore pay a transaction cost of $\tau-1>0$ for the exchange of currencies. For simplicity, we continue to assume that government transfers are not subject transaction costs, and therefore, maintain the identity $T+\varepsilon T^{*}=0$. Finally, the currency area is free of such transaction costs so that $\tau=1$. We therefore just need to discuss the flexible exchange rate regime.

In the presence of transaction costs, the Home consumer price of a Foreign variety $v^{*}$ is $p\left(v^{*}\right)=$ $\varepsilon \tau p^{*}\left(v^{*}\right)$ and the Foreign consumer price of Home variety $v$ is $p^{*}(v)=\tau p(v) / \varepsilon$. Thus, price indices depend on $\tau$ and $B(\epsilon)$ and $B^{*}(\varepsilon)$, given in equation (2), need to be rewritten as:

$$
B(\varepsilon)=\left(\frac{1}{2} b+\frac{1}{2} b^{*} \tau^{1-\sigma} \varepsilon^{1-\sigma}\right)^{\frac{1}{1-\sigma}}, \quad \text { and } \quad B^{*}(\varepsilon)=\left(\frac{1}{2} b^{*}+\frac{1}{2} b \tau^{1-\sigma} \varepsilon^{\sigma-1}\right)^{\frac{1}{1-\sigma}}
$$

Symmetry again implies $W=W^{*}$. It remains true that $Y+T=1$. Using the labor market clearing conditions, the exchange rate $\varepsilon$ is a function of the transfer $T$ and transaction cost $\tau$, which solves the following equation (see details in Appendix E):

$$
\frac{1-T}{1+\frac{T}{\varepsilon}}=\frac{b}{b^{*}} \varepsilon^{\sigma}\left(\frac{1+\tau^{1-\sigma} \varepsilon \Upsilon(\varepsilon, \tau)}{\tau^{1-\sigma}+\varepsilon \Upsilon(\varepsilon, \tau)}\right)
$$

where $\Upsilon(\varepsilon, \tau) \equiv\left(P /\left(\varepsilon P^{*}\right)\right)^{1-\sigma}=\left(B(\varepsilon) /\left(\varepsilon B^{*}(\varepsilon)\right)\right)^{1-\sigma}$ is a welfare-based measure of the real exchange rate. As expected, for $\tau=1, \Upsilon(\varepsilon, 1)=1$ and the above equation is equivalent to equation (15). With the appropriate modification described above, the wages $W$ and utility $U_{s}(T, W)$ are still determined by the expressions (17) and (18) and all other equilibrium conditions remain unchanged.

One expects that consumption falls with transaction costs. It is easy to show that this is true when shocks and transaction costs are small enough. Indeed, we know that consumption falls with 
higher price index and lower wage, which depend on $B(\varepsilon)$ and $\varepsilon$. Transaction costs have a positive effect on $B(\varepsilon)$ and $B^{*}(\varepsilon)$ but only a second-order effect on the exchange rate (that is, $\partial B(\varepsilon) / \partial \tau>0$ and $\left.\lim _{\tau \rightarrow 1, b / b^{*} \rightarrow 1} \mathrm{~d} \varepsilon / \mathrm{d} \tau=0\right)$. Hence, a small increase in $\tau$ from $\tau=1$ raises the price indices and reduces the wage because $B(\varepsilon)$ and $\mathrm{E}\left[(B(\varepsilon) A)^{\gamma-1}\right]$ increase (see (17)). As a result, transaction costs lead to first-order reduction of expected utility in the flexible exchange rate regime and make the currency areas with consumption sharing the better option. Figure 4 depicts the sets of parameters for which currency areas with consumption sharing transfers dominate flexible exchange rate regimes with no transfers. It clearly shows that currency areas become beneficial for a larger set of economic parameters and in particular for small enough shocks. The figure is plotted for a small transaction cost, $\tau=0.01$. To sum up, currency areas are more likely to be preferred in the presence of transaction costs.

We now discuss whether transaction costs make the transfer mechanisms more or less sustainable. The effect on the sustainability of transfers is difficult to disentangle because transaction costs reduce households' utility in all states of nature and at all levels of transfers. In other words, both benefits of sustaining transfers and of deviating from the mechanism fall with transaction costs. Since the sustainability conditions (22) are expressed in terms of utility differences, one may conjecture that the effect of transaction costs on these conditions is small. ${ }^{37}$ This conjecture turns out to be true in the simulation exercises we have computed. Overall, transaction costs have only small effects on the critical discount factors $\underline{\delta}^{f}$ and $\bar{\delta}^{f}$. Figure 5 plots those discount factors for the same parameters as in Figure 2 but for the set of transaction costs $\tau \in\{1.00,1.05,1.10,1.15\}$. Note that to show the small effect we need to use transaction costs that are much larger than one in Figure $4(\tau=1.01)$. For larger transaction costs, the critical discount factor $\underline{\delta}^{f}$ (thin dashed curves) slightly moves down while the critical discount factor $\bar{\delta}^{f}$ moves up (thin curves). This means that transaction costs make consumption sharing transfers slightly less sustainable. They nevertheless allow countries to sustain small transfers for a greater range of parameter values. To sum up, transaction costs have only a small effect on the sustainability of transfers under flexible exchange rates. It is still possible to find configuration (such as the point A) that sustains no transfer under flexible rates and sustain full consumption sharing under currency areas.

\footnotetext{
${ }^{37}$ In the presence of transaction costs, the consumption sharing transfers are no longer identical under fixed and flexible exchange rates. On the one hand, more resources are transferred to maintain the consumption of the country with the bad shock. On the other hand, transaction costs affect the exchange rates. We have taken this into account in our numerical exercise.
} 
EUc-EUf with transaction costs various $\psi$ and $\gamma$

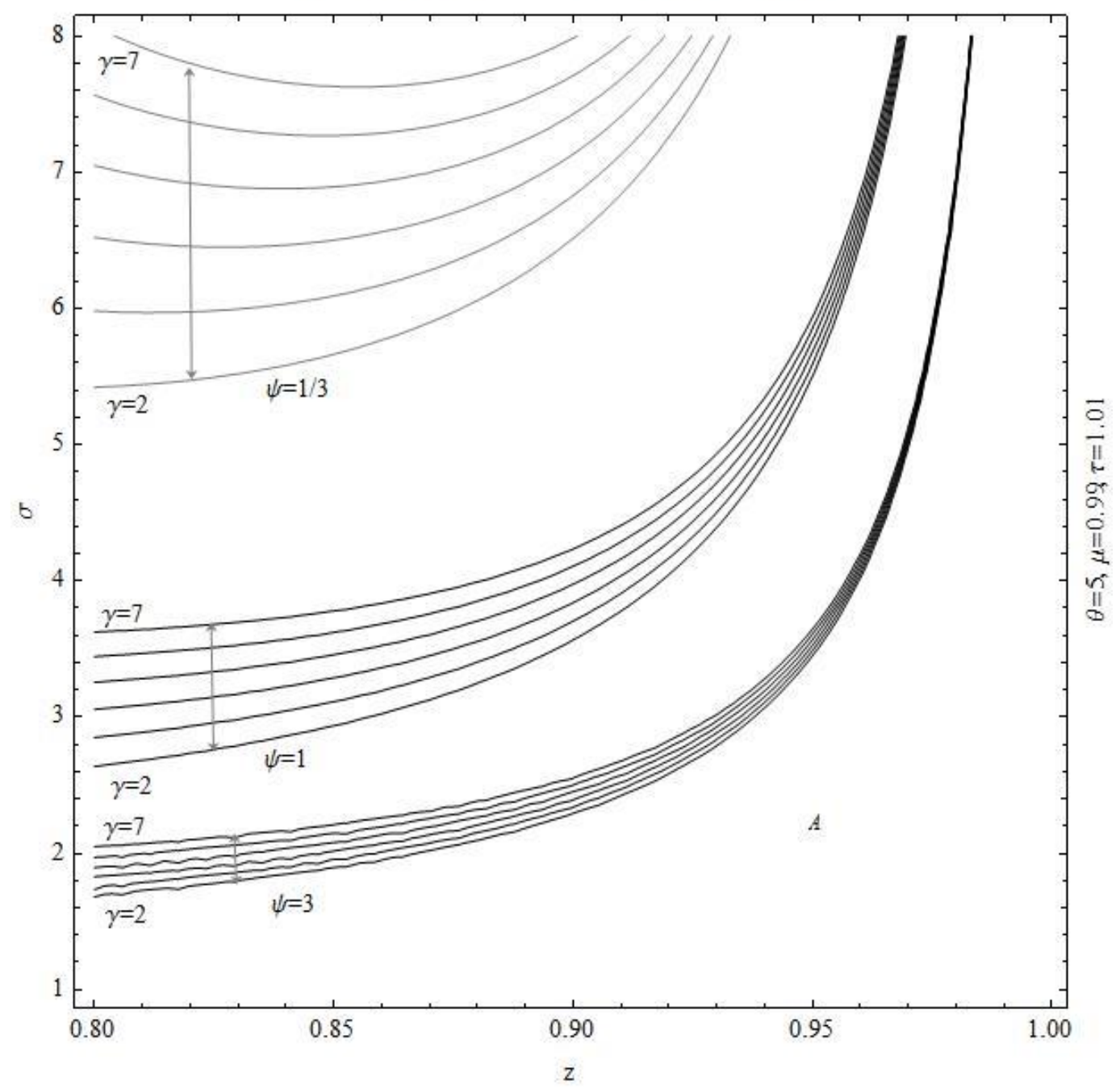

Figure 4: EXPECTED UTILITy With TRANSACTION COSTS: LOCI OF INDIFFERENCE BETWEEN A COMMON CURRENCY WITH CONSUMPTION SHARING TRANSFERS AND A FLEXIBLE EXCHANGE RATE REGIME WITH NO TRANSFERS FOR A RANGE OF VALUES OF $\psi$ AND $\gamma$. Other Parameter values: $\tau=1.1, \theta=5$ AND $\mu=0.99$.

\section{Conclusion}

This paper has discussed the relationship between the formation of a currency area and voluntary transfers between countries, where transfers are sustained by future risk sharing gains. A currency area can sustain more risk-sharing because the cost of reneging on transfers is higher in the currency area than in the flexible exchange rate regime. In this sense, the formation of a currency area is a commitment device that may allow countries to share more risk. The extent to which this extra risk sharing compensates for the inflexibility of the exchange rate is found to depend on parameter values: the desirability a currency area is enhanced by stronger product differentiation, increased 


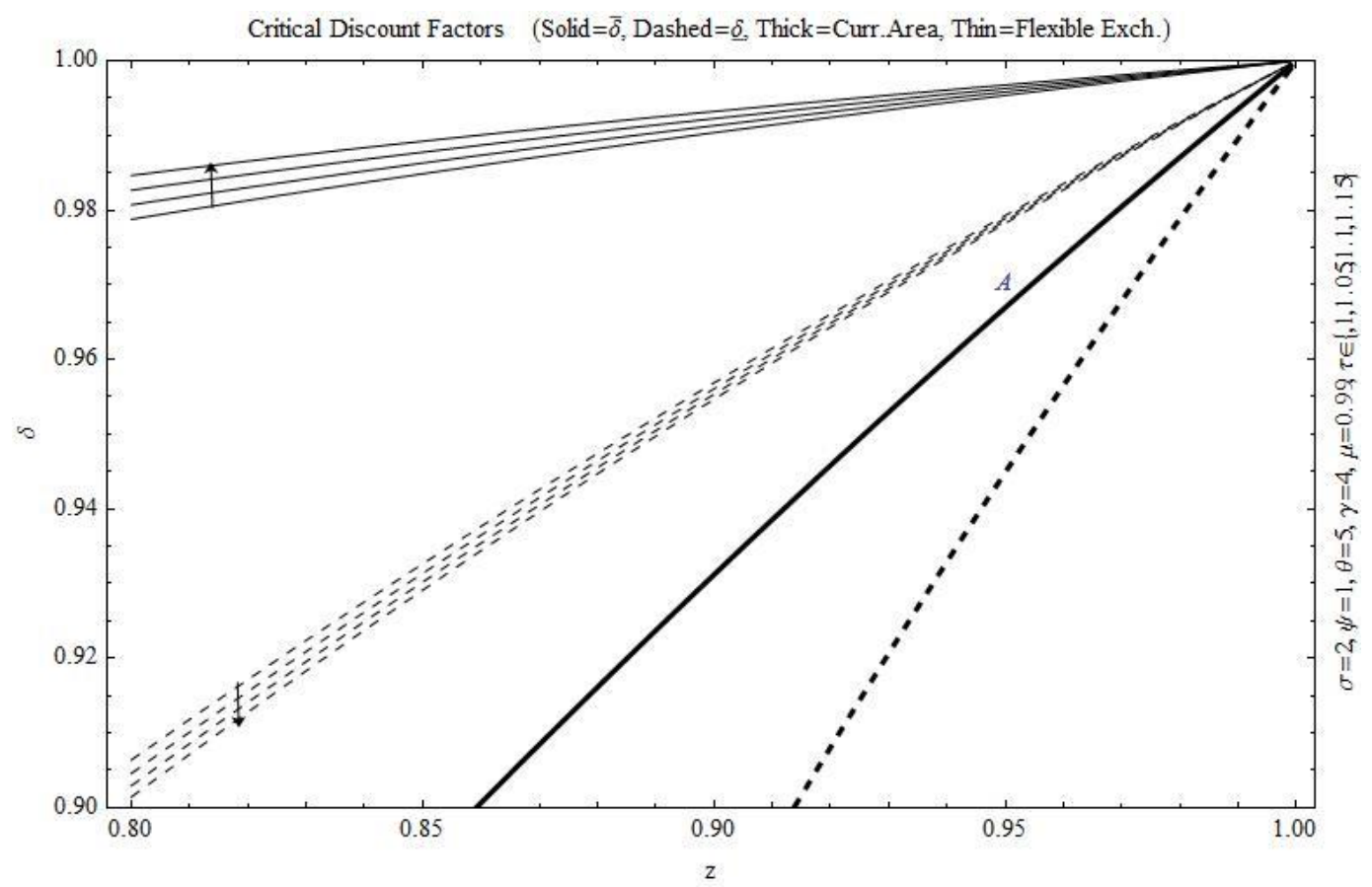

Figure 5: Critical discount FaCtors as A FunCtion of The SHOCK $z$. Lines ARE DRAWN FOR $\tau=1$ (NO TRANSACTION COSTS) AND $\tau=1.05,1.10,1.15$. ThE SOLID THIN LINES CORRESPOND TO $\bar{\delta}^{f}$, WHICH INCREASES WITH $\tau$ FOR EACH VALUE OF $z$. The DASHED THIN LINES CORRESPOND TO $\underline{\delta}^{f}$, WHICH FALLS WITH $\tau$. THE SOLID AND DASH THICK LINES CORRESPOND TO $\bar{\delta}^{c}$ AND $\underline{\delta}^{c}$ AND ARE UNAFFECTED BY $\tau$. Other PARAMETER VALUES: $\sigma=2, \gamma=5, \psi=1, \theta=5$ AND $\mu=0.99$.

labor supply flexibility and greater risk aversion.

It is important to recognize that our paper abstracts from a number of key features. First, we have restricted the analysis to two countries with symmetric and perfectly negatively correlated shocks. Second, we have assumed away any mechanism for intertemporal redistribution other than transfers. Both these assumptions have been made to keep the analysis tractable and to make the point that a currency area may have the benefit of sustaining more risk sharing than the flexible exchange rate regime. Despite the fact that our results are obtained in a specific context, our conclusion is that the increased potential for risk sharing that the currency area can sustain should not be ignored in the calculation of an optimal currency regime.

\section{References}

Alesina, A., Barro, R.J., 2002. Currency unions. The Quarterly Journal of Economics 117, 409-436. Alesina, A., Perotti, R., Spolaore, E., 1995. Together or separately? Issues on the costs and benefits 
of political and fiscal unions. European Economic Review 39, 751-758.

Arellano, C., Heathcote, J., 2010. Dollarization and financial integration. Journal of Economic Theory $145,944-973$.

Backus, D.K., Kehoe, P.J., Kydland, F.E., 1992. International real business cycles. Journal of Political Economy 100, 745-775.

Basu, S., Fernald, J.G., 1997. Returns to scale in U.S. production: Estimates and implications. Journal of Political Economy 105, 249-283.

Baxter, M., Jermann, U.J., 1997. The international diversification puzzle is worse than you think. American Economic Review 87, 170-180.

Bayoumi, T.A., 1994. A formal theory of optimum currency areas. IMF Staff Papers 42, 537-554.

Castro, R., Koumtingué, N., 2014. On the individual optimality of economic integration. Journal of Monetary Economics 68, 115-135.

Ching, S., Devereux, M.B., 2003. Mundell revisited: a simple approach to the costs and benefits of a single currency area? Review of International Economics 11, 674-691.

Cooper, R., Kempf, H., 2004. Overturning Mundell: Fiscal policy in a monetary union. The Review of Economic Studies 71, 371-396.

Corsetti, G., Dedola, L., Leduc, S., 2008. International risk sharing and the transmission of productivity shocks. Review of Economic Studies 75, 443-473.

Corsetti, G., Dedola, L., Leduc, S., 2010. Optimal monetary policy in open economies, in: Friedman, B.M., Woodford, M. (Eds.), Handbook of Monetary Economics. Elsevier. volume 3 of Handbook of Monetary Economics. chapter 16, pp. 861-933.

Corsetti, G., Martin, P., Pesenti, P., 2013. Varieties and the transfer problem. Journal of International Economics 89, 1-12.

Devereux, M.B., 2004. Should the exchange rate be a shock absorber? Journal of International Economics 62, 359-377.

Drèze, J.H., 2000. Economic and social securtiy in the twenty-first century, with attention to Europe. Scandinavian Journal of Economics 102, 327-348.

Farhi, E., Werning, I., 2014. Fiscal unions. Mimeo. 
Forni, M., Reichlin, L., 1999. Risk and potential insurance in Europe. European Economic Review 43, $1237-1256$.

French, K.R., Poterba, J.M., 1991. Investor diversification and international equity markets. American Economic Review 81, 170-180.

Fuchs, W., Lippi, F., 2006. Monetary union with voluntary participation. Review of Economic Studies 73, 437-457.

Kehoe, T.J., Levine, D.K., 2001. Liquidity constrained markets versus debt constrained markets. Econometrica 69, 575-598.

Kenen, P.B., 1969. The theory of optimum currency areas: An eclectic view, in: Mundell, R.A., Swoboda, A.K. (Eds.), Monetary Problems of the International Economy. Chicago University Press, Chicago, pp. 41-60.

Lewis, K.K., 1999. Trying to explain home bias in equities and consumption. Journal of Economic Literature 37, 571-608.

Mendoza, E.G., 1991. Real business cycles in a small open economy. American Economic Review $81,797-818$.

Mundell, R.A., 1961. A theory of optimum currency areas. American Economic Review 51, 657-665.

Mundell, R.A., 1973. Uncommon arguments for common currencies, in: Johnson, H.G., Swoboda, A.K. (Eds.), The Economics of Common Currencies. George Allen and Unwin, London. Proceedings of the Madrid Conference on Optimum Currency Areas, pp. 195-220.

Obstfeld, M., Rogoff, K., 1995. Exchange rate dynamics redux. Journal of Political Economy 103, 624-660.

Persson, T., Tabellini, G., 1996. Federal fiscal constitutions: Risk sharing and moral hazard. Econometrica 64, 623-646.

Reichling, F., Whalen, C., 2015. Estimates of the Frisch elasticity of labor supply: A review. Eastern Economic Journal, Forthcoming.

Rose, A.K., Engel, C., 2002. Currency unions and international integration. Journal of Money, Credit and Banking 34, 1067-89.

Samuelson, P.A., 1954. The transfer problem and transport costs, II: Analysis of effects of trade impediments. Economic Journal 62, 264-289. 
Stockman, A.C., Tesar, L.L., 1995. Tastes and technology in a two-country model of the business cycle: Explaining international comovements. American Economic Review 85, 168-185.

Thomas, J.P., Worrall, T., 1988. Self-enforcing wage contracts. Review of Economic Studies 55, $541-554$.

Todter, K.H., 2008. Estimating the uncertainty of relative risk aversion. Applied Financial Economics Letters 4, 25-27.

\section{Appendix A Wage setting}

This appendix derives the wage conditions given in equations (13) and (17). Recall that wages are set one period in advance taking aggregate profits, prices, money supply and transfers as given.

\section{Currency area}

The first-order condition for the household wage setting problem is

$$
w(h)=\frac{\theta}{\theta-1} \frac{\mathrm{E}\left[\ell_{s}(h)^{1+\psi}\right]}{\mathrm{E}\left[\xi V^{\prime}\left(\xi \frac{w(h) \ell_{s}(h)+\Pi_{s}+T_{s}+M_{0}}{P_{s}}\right) \frac{\ell_{s}(h)}{P_{s}}\right]} .
$$

Aggregating over households, the common wage $W$ satisfies

$$
W=\frac{\theta}{\theta-1} \frac{\mathrm{E}\left[L_{s}(W)^{1+\psi}\right]}{\mathrm{E}\left[\xi V^{\prime}\left(X_{s}\left(T_{s}, W\right)\right) \frac{L_{s}(W)}{P_{s}(W)}\right]} .
$$

Since $L_{s}(W)=\rho b_{s} / W$, we have

$$
\mathrm{E}\left[L_{s}(W)^{1+\psi}\right]=\frac{1}{\left(\frac{W}{\rho}\right)^{1+\psi}} \mathrm{E}\left[b_{s}^{(1+\psi)}\right]
$$

and since $V^{\prime}(x)=x^{-\gamma}, Y_{s}(W)=b_{s}, P_{s}(W)=(W / \rho) A_{s}$ and $M_{0}=(1-\mu) / \mu$, we have

$$
\mathrm{E}\left[\xi V^{\prime}\left(X_{s}\left(T_{s}, W\right)\right) \frac{L_{s}(W)}{P_{s}(W)}\right]=\frac{1}{\left(\frac{W}{\rho}\right)^{2-\gamma}} \xi^{1-\gamma} \mu^{\gamma} \mathrm{E}\left[\frac{\left(\mu\left(b_{s}+T_{s}\right)+(1-\mu)\right)^{-\gamma} b_{s}}{A_{s}^{1-\gamma}}\right] .
$$

Hence, the wage is set such that

$$
W=\left(\frac{W}{\rho}\right)^{1-\gamma-\psi} \frac{\theta}{\theta-1} \xi^{\gamma-1} \mu^{-\gamma} \frac{\mathrm{E}\left[b_{s}^{(1+\psi)}\right]}{\mathrm{E}\left[\left(\mu\left(b_{s}+T_{s}\right)+(1-\mu)\right)^{-\gamma} b_{s} A_{s}^{\gamma-1}\right]},
$$

or rewriting

$$
W=\left(\kappa_{0} \frac{\mathrm{E}\left[b_{s}^{(1+\psi)}\right]}{\mathrm{E}\left[\left(\mu\left(b_{s}+T_{s}\right)+(1-\mu)\right)^{-\gamma} b_{s} A_{s}^{\gamma-1}\right]}\right)^{\frac{1}{\gamma+\psi}}
$$


where $\kappa_{0}$ is a constant given by

$$
\kappa_{0}=\frac{\theta}{\theta-1} \rho^{\gamma+\psi-1} \xi^{\gamma-1} \mu^{-\gamma}
$$

Equation (A.3) is equation (13) in the paper. We note that

$$
\xi^{1-\gamma} \mu^{\gamma-1}\left(\frac{W}{\rho}\right)^{\gamma+\psi}=\kappa_{2} \frac{\mathrm{E}\left[b_{s}^{(1+\psi)}\right]}{\mathrm{E}\left[\left(\mu\left(b_{s}+T_{s}\right)+(1-\mu)\right)^{-\gamma} b_{s} A_{s}^{\gamma-1}\right]},
$$

where

$$
\kappa_{2}=\frac{\theta}{\theta-1} \frac{1}{\mu \rho} .
$$

Absent any transfers, $T_{s}=0$ for all $s$, the wage, denoted by $W_{0}$ satisfies

$$
W_{0}=\left(\kappa_{0} \frac{\mathrm{E}\left[b_{s}^{(1+\psi)}\right]}{\mathrm{E}\left[\left(\mu b_{s}+(1-\mu)\right)^{-\gamma} b_{s} A_{s}^{\gamma-1}\right]}\right)^{\frac{1}{\gamma+\psi}} .
$$

Using the consumption equalizing transfers $\bar{T}_{s}=(1 / 2)\left(b_{s}^{*}-b_{s}\right)$ given in equation (19), we get $\left(b_{s}+\bar{T}_{s}\right)=1$ because $b_{s} / 2+b_{s}^{*} / 2=1$. The wage in in this case, denoted by $\bar{W}$, is

$$
\bar{W}=\left(\kappa_{0} \frac{\mathrm{E}\left[b_{s}^{1+\psi}\right]}{\mathrm{E}\left[b_{s} A_{s}^{\gamma-1}\right]}\right)^{\frac{1}{\gamma+\psi}}=W_{0}\left(\frac{\mathrm{E}\left[\left(\mu b_{s}+(1-\mu)\right)^{-\gamma} b_{s} A_{s}^{\gamma-1}\right]}{\mathrm{E}\left[b_{s} A_{s}^{\gamma-1}\right]}\right)^{\frac{1}{\gamma+\psi}}
$$

which confirms equation (20) in the text.

For no transfers $\left(T_{s}=0\right)$ and no uncertainty where $a=a^{*}=1$ we get $b_{i}=1$ and $A=2^{1 /(1-\sigma)}$ and hence the wage is

$$
W=\left(\kappa_{0}\left(2^{\frac{\gamma-1}{\sigma-1}}\right)\right)^{\frac{1}{\gamma+\psi}} .
$$

Utility in state $s$ with transfer $T$ and wage $W$ is given by

$$
U_{s}(T, W)=\left(\frac{W}{\rho}\right)^{-(1+\psi)}\left(\frac{\xi^{1-\gamma} \mu^{\gamma-1}\left(\frac{W}{\rho}\right)^{\gamma+\psi}\left(\mu\left(b_{s}+T\right)+(1-\mu)\right)^{1-\gamma} A_{s}^{\gamma-1}}{1-\gamma}-\frac{b_{s}^{1+\psi}}{1+\psi}\right)
$$

Using (A.4) and abusing notation, this can be rewritten as

$$
U_{s}(T, W)=-\left(\frac{W}{\rho}\right)^{-(1+\psi)}\left(\frac{\kappa_{1} \mathrm{E}\left[b_{s}^{1+\psi}\right]}{1+\psi}\right)\left(\frac{\left(\mu\left(b_{s}+T\right)+(1-\mu)\right)^{1-\gamma} A_{s}^{\gamma-1}}{\mathrm{E}\left[\left(\mu\left(b_{s}+T_{s}\right)+(1-\mu)\right)^{-\gamma} b_{s} A_{s}^{\gamma-1}\right]}+\frac{b_{s}^{1+\psi}}{\kappa_{1} \mathrm{E}\left[b_{s}^{1+\psi}\right]}\right),
$$

where $\kappa_{1}=\kappa_{2}(1+\psi) /(\gamma-1)$ and $W$ is given by equation (A.3).

\section{Flexible exchange rates}

Using (16), we have

$$
\mathrm{E}\left[L\left(T_{s}, W\right)^{1+\psi}\right]=\frac{1}{\left(\frac{W}{\rho}\right)^{1+\psi}} \mathrm{E}\left[\left(1-T_{s}\right)^{(1+\psi)}\right]
$$


Since $V^{\prime}(x)=x^{-\gamma}, X_{s}(W, \varepsilon)=\xi /\left(\mu P_{s}(W, \varepsilon)\right), L(T, W)=(\rho / W)(1-T), P_{s}(W, \varepsilon)=(W / \rho) A_{s} B_{s}(\varepsilon)$ and $M_{0}=(1-\mu) / \mu$, we have

$$
\mathrm{E}\left[\xi V^{\prime}\left(X_{s}\left(W, \varepsilon_{s}\right)\right) \frac{L\left(T_{s}, W\right)}{P_{s}\left(W, \varepsilon_{s}\right)}\right]=\xi^{1-\gamma}\left(\frac{w}{\rho}\right)^{\gamma-2} \mu^{\gamma} \mathrm{E}\left[\left(A_{s} B_{s}\left(\varepsilon_{s}\right)\right)^{\gamma-1}\left(1-T_{s}\right)\right] .
$$

Substituting into (A.1), and recalling that $\varepsilon_{s}$ is a function of $T_{s}$ from equation (15), we get

$$
W=\left(\kappa_{0} \frac{\mathrm{E}\left[\left(1-T_{s}\right)^{(1+\psi)}\right]}{\mathrm{E}\left[\left(A_{s} B_{s}\left(\varepsilon_{s}\left(T_{s}\right)\right)\right)^{\gamma-1}\left(1-T_{s}\right)\right]}\right)^{\frac{1}{\gamma+\psi}} .
$$

Equation (A.8) reproduces equation (17) in the text. We note for future reference that

$$
\xi^{1-\gamma} \mu^{\gamma-1}\left(\frac{W}{\rho}\right)^{\gamma+\psi}=\kappa_{2} \frac{\mathrm{E}\left[\left(1-T_{s}\right)^{(1+\psi)}\right]}{\mathrm{E}\left[\left(A_{s} B_{s}\left(\varepsilon_{s}\left(T_{s}\right)\right)\right)^{\gamma-1}\left(1-T_{s}\right)\right]} .
$$

When $T_{s}=0$ for all $s$, we get

$$
W_{0}=\left(\kappa_{0} \frac{1}{\mathrm{E}\left[\left(A_{s} B_{s}\left(\varepsilon_{s}(0)\right)\right)^{\gamma-1}\right]}\right)^{\frac{1}{\gamma+\psi}}
$$

At $T_{s}=\bar{T}_{s}, \varepsilon_{s}\left(\bar{T}_{s}\right)=1, B_{s}(1)=1$ and $b_{s}+\bar{T}_{s}=1$. Hence, when $T_{s}=\bar{T}_{s}$ for all $s$,

$$
\bar{W}=\left(\kappa_{0} \frac{\mathrm{E}\left[b_{s}^{1+\psi}\right]}{\mathrm{E}\left[b_{s} A_{s}^{\gamma-1}\right]}\right)^{\frac{1}{\gamma+\psi}}=W_{0}\left(\frac{\mathrm{E}\left[\left(A_{s} B_{s}\left(\varepsilon_{s}(0)\right)\right)^{\gamma-1}\right]}{\mathrm{E}\left[b_{s} A_{s}^{\gamma-1}\right]}\right)^{\frac{1}{\gamma+\psi}}
$$

which again confirms equation (20) in the text.

If there are no transfers and no uncertainty $\left(a=a^{*}\right)$, we have $\varepsilon=1$ and hence, $A_{i}=1$. Therefore, in this case (with $a=1$ ),

$$
W=\left(\kappa_{0}\left(2^{\frac{\gamma-1}{\sigma-1}}\right)\right)^{\frac{1}{\gamma+\psi}} .
$$

This is the same as in the common currency case.

Utility in state $s$ with transfer $T$ and wage $W$ is given by

$$
U_{s}(T, W)=\left(\frac{W}{\rho}\right)^{-(1+\psi)}\left(\frac{\xi^{1-\gamma} \mu^{\gamma-1}\left(\frac{W}{\rho}\right)^{\gamma+\psi}\left(A_{s} B_{s}\left(\varepsilon_{s}(T)\right)\right)^{\gamma-1}}{1-\gamma}-\frac{(1-T)^{1+\psi}}{1+\psi}\right)
$$

Using (A.9), this can be rewritten as

$$
U_{s}(T, W)=-\left(\frac{W}{\rho}\right)^{-(1+\psi)}\left(\frac{\kappa_{1} \mathrm{E}\left[\left(1-T_{s}\right)^{1+\psi}\right]}{1+\psi}\right)\left(\frac{\left(A_{s} B_{s}\left(\varepsilon_{s}(T)\right)\right)^{\gamma-1}}{\mathrm{E}\left[\left(A_{s} B_{s}\left(\varepsilon_{s}\left(T_{s}\right)\right)\right)^{\gamma-1}\left(1-T_{s}\right)\right]}+\frac{(1-T)^{1+\psi}}{\kappa_{1} \mathrm{E}\left[\left(1-T_{s}\right)^{1+\psi}\right]}\right)
$$

where $\kappa_{1}=\kappa_{2}(1+\psi) /(\gamma-1)$ and $W$ is given by equation (A.8). 


\section{Appendix B Fiscal transfers}

In this appendix we compare a currency area with consumption equalising transfers to a flexible exchange rate regime with no transfers. Where necessary we use superscripts $c$ and $f$ to distinguish the two cases. Because of symmetry, we have $A_{s}=A$ for each $s$ and that $\mathrm{E}\left[b_{s}\right]=1$ in equation (20). Using this together with setting $T_{s}=0$ for each $s$ in equation (17) gives

$$
\bar{W}=\left(\kappa_{0} \frac{\mathrm{E}\left[b_{s}^{1+\psi}\right]}{A^{\gamma-1}}\right)^{\frac{1}{\gamma+\psi}}, \quad \text { and } \quad W_{0}^{f}=\left(\kappa_{0} \frac{1}{A^{\gamma-1} \mathrm{E}\left[B_{s}\left(\varepsilon_{s}(0)\right)^{\gamma-1}\right]}\right)^{\frac{1}{\gamma+\psi}}
$$

where $\bar{W}$ is the wage with consumption equalizing transfers and $W_{0}^{f}$ is the wage with no transfers in the flexible exchange rate regime. Hence, the ratio of the two wages is

$$
\left(\frac{W_{0}^{f}}{\bar{W}}\right)^{1+\psi}=\left(\frac{1}{\mathrm{E}\left[b_{s}^{1+\psi}\right] \mathrm{E}\left[B_{s}\left(\varepsilon_{s}(0)\right)^{\gamma-1}\right]}\right)^{\frac{1+\psi}{\gamma+\psi}}
$$

It can be shown this ratio is larger than one. Substituting $b_{s}+\bar{T}_{s}=1$ into equation (A.6), using equation (A.4) and taking expectations, the expected utility in the common currency (using superscript $c$ to denote the common currency regime) is

$$
\mathrm{E}\left[U_{s}^{c}\left(\bar{T}_{s}, \bar{W}\right)\right]=-\left(\frac{\bar{W}}{\rho}\right)^{-(1+\psi)}\left(\frac{1+\kappa_{1}}{1+\psi}\right) \mathrm{E}\left[b_{s}^{1+\psi}\right]
$$

Similarly, setting $T_{s}=0$ in equation (A.11), using equation (A.9) and taking expectations, the expected utility in the flexible regime (using superscript $f$ to denote the flexible exchange rate regime) is

$$
\mathrm{E}\left[U_{s}^{f}\left(0, W_{0}^{f}\right)\right]=-\left(\frac{W_{0}^{f}}{\rho}\right)^{-(1+\psi)}\left(\frac{1+\kappa_{1}}{1+\psi}\right) .
$$

We finally establish the conditions under which the currency area dominates. Taking the ratio and using equation (B.1) we have

$$
\frac{\mathrm{E}\left[U_{s}^{c}\left(\bar{T}_{s}, \bar{W}\right)\right]}{\mathrm{E}\left[U_{s}^{f}\left(0, W_{0}^{f}\right)\right]}=\left(\frac{W_{0}^{f}}{\bar{W}}\right)^{1+\psi} \mathrm{E}\left[b_{s}^{1+\psi}\right]=\left(\frac{1}{\mathrm{E}\left[B_{s}\left(\varepsilon_{s}(0)\right)^{\gamma-1}\right]}\right)^{\frac{1+\psi}{\gamma+\psi}}\left(\mathrm{E}\left[b_{s}^{1+\psi}\right]\right)^{\frac{\gamma-1}{\gamma+\psi}} .
$$

Let $r_{1}(z)$ denote this ratio as a function of the shock $z$ in the two-state case. Since utility is negative, $r_{1}(z)<1$ is equivalent to $\mathrm{E}\left[U_{s}^{c}\left(\bar{T}_{s}, \bar{W}\right)\right]>\mathrm{E}\left[U_{s}^{f}\left(0, W_{0}^{f}\right)\right]$. It is easy to check that $r_{1}(1)=1$ and $r_{1}^{\prime}(1)=0$. The sign of $r_{1}^{\prime \prime}(1)$ is the same as the sign of $\left(\psi \sigma^{2}-\sigma-\gamma\right)$. Thus, $r_{1}^{\prime \prime}(1)<0$ if $\gamma>\sigma(\psi \sigma-1)$. Hence, for $z$ close to one, the currency union dominates if $\gamma>\sigma(\psi \sigma-1)$. We have $\sigma>1$ and $\psi>0$. Likewise, it can be shown that $\lim _{z \rightarrow 0} r_{1}(z)=0$ and therefore, by the continuity of $r_{1}(z)$ in $z$, there is a $\hat{z}$ such that for $z<\hat{z}$ (that is, for large shocks) the currency area will dominate. This proves Proposition 1. 


\section{Appendix C Sustaining consumption sharing}

This appendix computes the critical discount factors that sustain consumption equalizing transfers and provides a prrof of Proposition 2.

Provided $\mathrm{E}\left[U_{s}\left(\bar{T}_{s}, \bar{W}\right)\right]-\mathrm{E}\left[U_{s}\left(0, W_{0}\right)\right]>0$, the inequality (24) shows that full consumption-sharing is sustainable for discount factors above a critical level $\bar{\delta}$. Define $\lambda:=(\delta-1) / \delta$ and $\bar{\lambda}:=(\bar{\delta}-1) / \bar{\delta}$ where

$$
\bar{\lambda}=\frac{\mathrm{E}\left[U_{s}\left(\bar{T}_{s}, \bar{W}\right)\right]-\mathrm{E}\left[U_{s}\left(0, W_{0}\right)\right]}{U_{G}\left(\bar{T}_{G}, \bar{W}\right)-U_{G}(0, \bar{W})}<0
$$

Then, full consumption-sharing is possible if $\lambda \geq \bar{\lambda}$. Using the Maclaurin series, we have the approximation $\bar{\delta} \approx 1+\bar{\lambda}$ for $\bar{\lambda}$ close to zero.

\section{Common currency}

Let

$$
\chi_{1}(\mu):=\frac{\mathrm{E}\left[\left(\mu b_{s}+(1-\mu)\right)^{1-\gamma}\right]}{\mathrm{E}\left[\left(\mu b_{s}+(1-\mu)\right)^{-\gamma} b_{s}\right]} .
$$

With $\mathrm{E}\left[b_{s}\right]=1$, it is easily checked that $\chi_{1}(0)=\chi_{1}(1)=1$. Also, since either $b_{s}>\mu b_{s}+(1-\mu)>1$ or $b_{s}<\mu b_{s}+(1-\mu)<1$ for $\mu \in(0,1)$, it is easily checked that $\chi_{1}(\mu)>1$ for $\mu \in(0,1)$. Using equations (A.6) and (A.4), $A_{s}=A, \mathrm{E}\left[b_{s}\right]=1, b_{s}+\bar{T}_{s}=1$, and taking expectations

$$
\begin{aligned}
& \mathrm{E}\left[U_{s}\left(\bar{T}_{s}, \bar{W}\right)\right]=-\left(\frac{\bar{W}}{\rho}\right)^{-(1+\psi)}\left(\frac{1+\kappa_{1}}{1+\psi}\right) \mathrm{E}\left[b_{s}^{1+\psi}\right], \\
& \mathrm{E}\left[U_{s}\left(0, W_{0}\right)\right]=-\left(\frac{W_{0}}{\rho}\right)^{-(1+\psi)}\left(\frac{1+\chi_{1}(\mu) \kappa_{1}}{1+\psi}\right) \mathrm{E}\left[b_{s}^{1+\psi}\right] .
\end{aligned}
$$

From equation (A.2),

$$
\bar{W}=W_{0}\left(\mathrm{E}\left[\left(\mu b_{s}+(1-\mu)\right)^{-\gamma} b_{s}\right]\right)^{\frac{1}{\gamma+\psi}} .
$$

It is clear that $\bar{W}>W_{0}$ for $\mu=1$ because of the convexity of $b_{s}^{1-\gamma}$. Equally, $\bar{W}=W_{0}$ for $\mu=0$. It can also be checked that $\bar{W}<W_{0}$ for $\mu$ small enough. Thus, we conclude that $\bar{W}>W_{0}$ for $\mu>\hat{\mu}$ where $0<\hat{\mu}<1$. Therefore, since $\chi_{1}(\mu) \geq 1, \mathrm{E}\left[U_{s}\left(\bar{T}_{s}, \bar{W}\right)\right]>\mathrm{E}\left[U_{s}\left(0, W_{0}\right)\right]$ for $\mu>\hat{\mu}$. Also, using equations (A.6) and (A.4)

$$
U_{G}\left(\bar{T}_{G}, \bar{W}\right)-U_{G}(0, \bar{W})=-\left(\frac{\bar{W}}{\rho}\right)^{-(1+\psi)}\left(\frac{\kappa_{1} \mathrm{E}\left[b_{s}^{1+\psi}\right]}{1+\psi}\right)\left(1-\left(\mu b_{G}+(1-\mu)\right)^{1-\gamma}\right) .
$$

Substituting the expressions for $\mathrm{E}\left[U_{s}\left(\bar{T}_{s}, \bar{W}\right)\right], \mathrm{E}\left[U_{s}\left(0, W_{0}\right)\right]$ and $U_{G}\left(\bar{T}_{G}, \bar{W}\right)-U_{G}(0, \bar{W})$ into (C.1) and using equation (C.2) gives

$$
\bar{\lambda}=\frac{\left(1+\frac{1}{\kappa_{1}}\right)-\mathrm{E}\left[\left(\mu b_{s}+(1-\mu)\right)^{-\gamma} b_{s}\right]^{\frac{1+\psi}{\gamma+\psi}}\left(\chi_{1}(\mu)+\frac{1}{\kappa_{1}}\right)}{1-\left(\left(\mu b_{G}+(1-\mu)\right)^{1-\gamma}\right)} .
$$




\section{Small shocks and derivation of equation (25)}

For small risks ( $z$ close to one), the above expression can be linearized to give (where the superscript $c$ indicates it is the critical value in the currency area case)

$$
\bar{\lambda}^{c} \approx \frac{1}{4}\left(\left(1+\frac{1}{\kappa_{1}}\right)-(1-\mu)\left(\frac{(\psi-1)}{(1+\psi)}+\left(\frac{1}{\kappa_{1}}\right) \frac{(1+\gamma)}{(\gamma-1)}\right)\right)\left(\frac{1+\psi}{\gamma+\psi}\right) \gamma(\sigma-1)(z-1) .
$$

Using $1 / \kappa_{1}=(\theta-1)(\gamma-1) \mu(\sigma-1) /(1+\psi) \theta \sigma$ and letting $\mu \rightarrow 1$ gives

$$
\bar{\lambda}^{c} \approx \frac{1}{4}\left(\sigma(1+\psi)+(\gamma-1)(\sigma-1) \frac{(\theta-1)}{\theta}\right)\left(\frac{\gamma}{\gamma+\psi}\right)\left(\frac{\sigma-1}{\sigma}\right)(z-1) .
$$

For $z$ close to one, $\bar{\lambda}^{c}$ is close to zero and hence, using the approximation $\bar{\delta} \approx 1+\bar{\lambda}$

$$
\bar{\delta}^{c} \approx 1+\frac{1}{4}\left(\sigma(1+\psi)+(\gamma-1)(\sigma-1) \frac{(\theta-1)}{\theta}\right)\left(\frac{\gamma}{\gamma+\psi}\right)\left(\frac{\sigma-1}{\sigma}\right)(z-1) .
$$

It can be seen, that since $z<1, \bar{\delta}^{c}<1$ for all parameter values. Setting $\psi=(\theta-1) / \theta=1$ gives equation (25) in the text.

\section{Flexible exchange rate}

Using equations (A.11) and (A.9), $A_{s}=A, \mathrm{E}\left[b_{s}\right]=1, b_{s}+\bar{T}_{s}=1, \varepsilon_{s}\left(\bar{T}_{s}\right)=1, B_{s}(1)=1$ and taking expectations

$$
\begin{aligned}
\mathrm{E}\left[U_{s}\left(\bar{T}_{s}, \bar{W}\right)\right] & =-\left(\frac{\bar{W}}{\rho}\right)^{-(1+\psi)}\left(\frac{1+\kappa_{1}}{1+\psi}\right) \mathrm{E}\left[b_{s}^{1+\psi}\right], \\
\mathrm{E}\left[U_{s}\left(0, W_{0}\right)\right] & =-\left(\frac{W_{0}}{\rho}\right)^{-(1+\psi)}\left(\frac{1+\kappa_{1}}{1+\psi}\right) .
\end{aligned}
$$

Therefore,

$$
\mathrm{E}\left[U_{s}\left(\bar{T}_{s}, \bar{W}\right)\right]-\mathrm{E}\left[U_{s}\left(0, W_{0}\right)\right]=-\left(\frac{\bar{W}}{\rho}\right)^{-(1+\psi)}\left(\frac{1+\kappa_{1}}{1+\psi}\right)\left(\mathrm{E}\left[b_{s}^{1+\psi}\right]-\left(\frac{\bar{W}}{W_{0}}\right)^{1+\psi}\right)
$$

Also, rewriting equation (B.1) gives

$$
\bar{W}=W_{0}\left(\mathrm{E}\left[b_{s}^{1+\psi}\right] \mathrm{E}\left[\left(B_{s}\left(\varepsilon_{s}(0)\right)\right)^{\gamma-1}\right]\right)^{\frac{1}{\gamma+\psi}}
$$

We have $\mathrm{E}\left[b_{s}^{1+\psi}\right] \geq 1$ by the convexity of $b_{s}^{1+\psi}$ with strict inequality for $\psi>0$ and $z<1$. Also, since $B_{s}\left(\varepsilon_{s}(0)\right)$ is a weighted power mean of 1 and $\varepsilon_{s}(0)$, we conclude that $B_{s}\left(\varepsilon_{s}(0)\right) \geq \min \left\{1, \varepsilon_{s}(0)\right\}$. With two states this gives $B_{G}\left(\varepsilon_{G}(0)\right)>\varepsilon_{G}(0)$ and $B_{B}\left(\varepsilon_{B}(0)\right)>\varepsilon_{B}(0)=1 / \varepsilon_{G}(0)>1$. For $\gamma>1, \varepsilon_{G}(0)^{\gamma-1}+\varepsilon_{G}(0)^{1-\gamma}$ is decreasing in $\varepsilon_{G}(0)$ and has a minimum value of 2 when $\varepsilon_{G}(0)=1$, which occurs when $z=1$. Thus, it can be concluded that $\mathrm{E}\left[\left(B_{s}\left(\varepsilon_{s}(0)\right)\right)^{\gamma-1}\right] \geq 1$ with strict inequality for $z<1$ and hence, $\bar{W}>W_{0}$ for $z<1$. Since the expected utility $\mathrm{E}\left[U_{s}\left(\bar{T}_{s}, \bar{W}\right)\right]$ is the same under both regimes, the conditions under which $\mathrm{E}\left[U_{s}\left(\bar{T}_{s}, \bar{W}\right)\right]-\mathrm{E}\left[U_{s}\left(0, W_{0}\right)\right]>0$ have already been established in Appendix B. In particular, if 
$\gamma>\sigma(\psi \sigma-1)$, then $\mathrm{E}\left[U_{s}\left(\bar{T}_{s}, \bar{W}\right)\right]-\mathrm{E}\left[U_{s}\left(0, W_{0}\right)\right]>0$ for small risks and there is always a risk large enough $\left(z\right.$ small enough) such that $\mathrm{E}\left[U_{s}\left(\bar{T}_{s}, \bar{W}\right)\right]-\mathrm{E}\left[U_{s}\left(0, W_{0}\right)\right]>0$. From equations (A.11) and (A.9)

$$
U_{G}\left(\bar{T}_{G}, \bar{W}\right)-U_{G}(0, \bar{W})=-\left(\frac{\bar{W}}{\rho}\right)^{-(1+\psi)}\left(\frac{\kappa_{1} \mathrm{E}\left[b_{s}^{1+\psi}\right]}{1+\psi}\right)\left(1-B_{G}\left(\varepsilon_{G}(0)\right)^{\gamma-1}+\frac{b_{G}^{1+\psi}-1}{\kappa_{1} \mathrm{E}\left[b_{s}^{1+\psi}\right]}\right) .
$$

Therefore, substituting the expressions for $\mathrm{E}\left[U_{s}\left(\bar{T}_{s}, \bar{W}\right)\right], \mathrm{E}\left[U_{s}\left(0, W_{0}\right)\right]$ and $U_{G}\left(\bar{T}_{G}, \bar{W}\right)-U_{G}(0, \bar{W})$ into (C.1) and using equation (C.3) gives

$$
\bar{\lambda}=\frac{\left(1+\frac{1}{\kappa_{1}}\right)\left(1-\left(\mathrm{E}\left[b_{s}^{1+\psi}\right]\right)^{\frac{1-\gamma}{\gamma+\psi}}\left(\mathrm{E}\left[B_{s}\left(\varepsilon_{s}(0)\right)^{\gamma-1}\right]\right)^{\frac{1+\psi}{\gamma+\psi}}\right)}{\left(1-B_{G}\left(\varepsilon_{G}(0)\right)^{\gamma-1}\right)+\frac{1}{\kappa_{1}}\left(b_{G}^{1+\psi}-1\right)\left(\mathrm{E}\left[b_{s}^{1+\psi}\right]\right)^{-1}}
$$

\section{Small shocks and derivation of equation (26)}

For small shocks, linearizing about $z=1$ gives the approximation (using the superscript $f$ to indicate the flexible exchange rate case)

$$
\bar{\lambda}^{f} \approx \frac{1}{4}\left(1+\frac{1}{\kappa_{1}}\right)\left(\frac{1+\psi}{\gamma+\psi}\right)\left(\frac{(\gamma-1)(\gamma-\sigma(\sigma \psi-1))}{(\gamma-1)+(1+\psi)\left(\frac{\sigma}{\kappa_{1}}\right)}\right)\left(\frac{\sigma-1}{\sigma}\right)(z-1) .
$$

Using $1 / \kappa_{1}=(\theta-1)(\gamma-1) \mu(\sigma-1) /(1+\psi) \theta \sigma$ and the approximation $\bar{\delta} \approx 1+\bar{\lambda}$, we get

$$
\bar{\delta}^{f} \approx 1+\frac{1}{4}\left(1+\mu \frac{\theta-1}{\theta} \frac{\gamma-1}{1+\psi} \frac{\sigma-1}{\sigma}\right)\left(\frac{1+\psi}{\gamma+\psi}\right)\left(\frac{(\gamma-\sigma(\sigma \psi-1))}{1+\mu(\sigma-1) \frac{(\theta-1)}{\theta}}\right)\left(\frac{\sigma-1}{\sigma}\right)(z-1) .
$$

It can be seen that for $\gamma<\sigma(\sigma \psi-1)$, the above approximation gives $\bar{\delta}^{f}>1$. Since this is not possible, we conclude $\bar{\delta}^{f}=1$ for $\gamma \leq \sigma(\sigma \psi-1)$ and $\bar{\delta}<1$ for $\gamma>\sigma(\sigma \psi-1)$. Setting $\mu=\psi=(\theta-1) / \theta=1$ in the above approximation gives equation (26) in the text.

\section{Proof of Proposition 2}

Recall that $\bar{\delta}:=1 /(1-\bar{\lambda})$ where $\bar{\lambda}<0$. Thus, $\bar{\delta}^{c}<\bar{\delta}^{f}$ implies that $\bar{\lambda}^{c}<\bar{\lambda}^{f}$ or equivalently $\bar{\lambda}^{c} / \bar{\lambda}^{f}>1$. For $z$ close to 1 , we have the ratio

$$
\frac{\bar{\lambda}^{c}}{\bar{\lambda}^{f}}=\left(1-(1-\mu)\left(\frac{\mu(1+\gamma)(\sigma-1)\left(\frac{\theta-1}{\theta}\right)+\sigma(\psi-1)}{\mu(\gamma-1)(\sigma-1)\left(\frac{\theta-1}{\theta}\right)+\sigma(1+\psi)}\right)\right)\left(\gamma \sigma \frac{\left(1+(\sigma-1)\left(\frac{\theta-1}{\theta}\right)\right)}{(\gamma-\sigma(\sigma \psi-1))}\right) .
$$

As argued above, under the condition $\gamma \leq \sigma(\sigma \psi-1)$, we have that $\bar{\delta}^{c}<1$ and $\bar{\delta}^{f}=1$ so that $\bar{\delta}^{c}<\bar{\delta}^{f}$. For the opposite condition $\gamma>\sigma(\sigma \psi-1)$, we focus on the case where $\mu=1$ and $z$ close to 1 . We get the ratio

$$
r_{2}:=\frac{\bar{\lambda}^{c}}{\bar{\lambda}^{f}}=\gamma \sigma \frac{\left(1+(\sigma-1)\left(\frac{\theta-1}{\theta}\right)\right)}{(\gamma-\sigma(\sigma \psi-1))}
$$

which is positive because the numerator and denominator are positive. It is easily checked that $r_{2}$ is decreasing in $\gamma$ for $\psi>1 / \sigma$. Also $\lim _{\gamma \rightarrow \infty} r_{2}=1+(\sigma-1)(1+\sigma((\theta-1) / \theta))>1$. Thus, $r_{2}>1$ for $\psi>1 / \sigma$ and hence, $\bar{\delta}^{c}<\bar{\delta}^{f}$. By continuity, the same result applies for $\mu$ close to one. 


\section{Appendix D Sustaining transfers}

This appendix computes the critical discount factors that just sustain small transfers. Some of the more tedious computations is relegated to a supplementary Appendix S.

\section{Currency area}

Recall that $\epsilon=1$ and therefore that the expected discounted utility in state $G$ is

$$
\begin{aligned}
V_{G}(\mathcal{T}):= & U_{G}(0, W(0))-U_{G}(0, W(\mathcal{T})) \\
& \quad+\frac{1}{(1-\delta)}\left(\left(1-\frac{\delta}{2}\right)\left(U_{G}(-\mathcal{T}, W(\mathcal{T}))-U_{G}(0, W(0))\right)+\left(\frac{\delta}{2}\right)\left(U_{B}(\mathcal{T}, W(\mathcal{T}))-U_{B}(0, W(0))\right)\right) .
\end{aligned}
$$

We can therefore rewrite the sustainability condition $V_{G}^{\prime}(0)>0$ as $\delta>\underline{\delta}$ where

$$
\frac{1}{\underline{\delta}}=\frac{\left(\mathrm{E}\left[\frac{\partial U_{s}(0, W(0))}{\partial T}\right]+\left(\frac{W^{\prime}(0)}{W(0)}\right) \mathrm{E}\left[\frac{\partial U_{s}(0, W(0))}{\partial W} W(0)\right]\right)}{\frac{\partial U_{G}(0, W(0))}{\partial T}} .
$$

We now consider each of the terms in (D.1). First, differentiating the wage equation (A.3) with $T_{G}=-\mathcal{T}$ and $T_{B}=\mathcal{T}$ and recalling that $A_{s}=A$, we compute

$$
\frac{W^{\prime}(0)}{W(0)}=-\frac{\mu \gamma}{\gamma+\psi} \frac{\left(\left(\mu b_{G}+(1-\mu)\right)^{-\gamma-1} b_{G}-\left(\mu b_{B}+(1-\mu)\right)^{-\gamma-1} b_{B}\right)}{\left(\left(\mu b_{G}+(1-\mu)\right)^{-\gamma} b_{G}+\left(\mu b_{B}+(1-\mu)\right)^{-\gamma} b_{B}\right)} .
$$

Next, recall that $L_{s}(W)=b_{s} \rho / W$ and with $M_{0}=(1-\mu) / \mu$ and $A_{s}=A, X_{s}\left(T_{s}, W\right)=\xi\left(\mu\left(b_{s}+T_{s}\right)+(1-\right.$ $\mu))(\rho /(\mu W A))$. We compute,

$$
W(0) \frac{\partial U_{s}(0, W(0))}{\partial W}=-\left(\frac{W(0)}{\rho}\right)^{-(1+\psi)} \kappa_{2} \mathrm{E}\left[b_{s}^{1+\psi}\right]\left(\frac{\left(\mu b_{s}+(1-\mu)\right)^{1-\gamma}}{\mathrm{E}\left[\left(\mu b_{s}+(1-\mu)\right)^{-\gamma} b_{s}\right]}-\frac{1}{\kappa_{2}} \frac{b_{s}^{1+\psi}}{\mathrm{E}\left[b_{s}^{1+\psi}\right]}\right)
$$

and

$$
\frac{\partial U_{s}(0, W(0))}{\partial T}=\mu\left(\frac{W(0)}{\rho}\right)^{-(1+\psi)} \kappa_{2} \mathrm{E}\left[b_{s}^{1+\psi}\right]\left(\frac{\left(\mu b_{s}+(1-\mu)\right)^{-\gamma}}{\mathrm{E}\left[\left(\mu b_{s}+(1-\mu)\right)^{-\gamma} b_{s}\right]}\right),
$$

where $\kappa_{2}=\theta /((\theta-1) \mu \rho)$. Therefore,

$$
\underline{\frac{1}{\delta}}=\frac{\mathrm{E}\left[\left(\mu b_{s}+(1-\mu)\right)^{-\gamma}\right]+\frac{\gamma}{\gamma+\psi}\left(\frac{\mathrm{E}\left[\left(\mu b_{s}+(1-\mu)\right)^{1-\gamma}\right]}{\mathrm{E}\left[\left(\mu b_{s}+(1-\mu)\right)^{-\gamma} b_{s}\right]}-\frac{1}{\kappa_{2}}\right)\left(\frac{1}{2}\left(\mu b_{G}+(1-\mu)\right)^{-\gamma-1}-\frac{1}{2}\left(\mu b_{B}+(1-\mu)\right)^{-\gamma-1}\right)}{\left(\mu b_{G}+(1-\mu)\right)^{-\gamma}} .
$$

\section{Small shocks and the derivation of equation (27)}

Taking a first-order approximation of this equation about $z=1$ gives

$$
\frac{1}{\underline{\delta}^{c}} \approx 1-\frac{1}{2}\left(\frac{\mu \psi}{(\gamma+\psi)}+\frac{\mu \gamma+(1-\mu)\left(\kappa_{2}-1\right)}{(\gamma+\psi) \kappa_{2}}\right) \gamma(\sigma-1)(z-1) .
$$


Note the term in the brackets is positive because $\kappa_{2}>1$. (This is equivalent to positive gains from risksharing). Hence, we can conclude that $\underline{\delta}^{c}<1$ for small shocks. Substituting for $\kappa_{2}$ gives

$$
\frac{1}{\underline{\delta}^{c}} \approx 1-\frac{1}{2}\left(\frac{1+\mu(\psi-1)}{(\gamma+\psi)}+\frac{\mu(\mu \gamma-(1-\mu))(\sigma-1)\left(\frac{\theta-1}{\theta}\right)}{(\gamma+\psi) \sigma}\right) \gamma(\sigma-1)(z-1) .
$$

Using $\underline{\lambda}:=(\underline{\delta}-1) / \underline{\delta}$ and the approximation $\underline{\delta} \approx 1+\underline{\lambda}$ and setting $\mu=\psi=(\theta-1) / \theta=1$ gives $(27)$ in the text.

\section{Flexible case}

Writing $W_{0}:=W(0)$ and $\epsilon_{0}:=\epsilon(0)$, we have

$$
\begin{aligned}
V_{G}(\mathcal{T}):= & U_{G}\left(0, W_{0}, \epsilon_{0}\right)-U_{G}\left(0, W(\mathcal{T}), \epsilon_{0}\right) \\
& +\frac{1}{(1-\delta)}\left(\left(1-\frac{\delta}{2}\right)\left(U_{G}(-\varepsilon(\mathcal{T}) \mathcal{T}, W(\mathcal{T}), \epsilon(\mathcal{T}))-U_{G}\left(0, W_{0}, \epsilon_{0}\right)\right)\right) \\
& +\frac{1}{(1-\delta)}\left(\left(\frac{\delta}{2}\right)\left(U_{B}(\mathcal{T}, W(\mathcal{T}), 1 / \epsilon(\mathcal{T}))-U_{B}\left(0, W_{0}, 1 / \epsilon_{0}\right)\right)\right)
\end{aligned}
$$

Therefore, the sustainability condition $V_{G}^{\prime}(0)>0$ requires that $\delta>\underline{\delta}^{f}$ where

$\frac{1}{\underline{\delta}^{f}}=\frac{\frac{1}{2}\left(\frac{\partial U_{B}\left(0, W_{0}, 1 / \epsilon_{0}\right)}{\partial T}+\frac{\partial U_{G}\left(0, W_{0}, \epsilon_{0}\right)}{\partial T} \epsilon_{0}-\left(\frac{\partial U_{B}\left(0, W_{0}, 1 / \epsilon_{0}\right)}{\partial \varepsilon}\left(\frac{1}{\epsilon_{0}^{2}}\right)+\frac{\partial U_{G}\left(0, W_{0}, \epsilon_{0}\right)}{\partial \varepsilon}\right) \epsilon^{\prime}(0)+\left(\frac{W^{\prime}(0)}{W_{0}}\right)\left(\frac{\partial U_{G}\left(0, W_{0}, \epsilon_{0}\right)}{\partial W} W_{0}+\frac{\partial U_{B}\left(0, W_{0}, 1 / \epsilon_{0}\right)}{\partial W} W_{0}\right)\right)}{\frac{\partial U_{G}\left(0, W_{0}, \epsilon_{0}\right)}{\partial T} \epsilon_{0}-\frac{\partial U_{G}\left(0, W_{0}, \epsilon_{0}\right)}{\partial \varepsilon} \epsilon^{\prime}(0)}$.

To evaluate this, we evaluate the components of this formula and substitute into (D.5). First, we have

$$
\begin{aligned}
U_{G}(-\epsilon(\mathcal{T}) \mathcal{T}, W, \epsilon) & =\frac{X_{G}(W, \epsilon)^{1-\gamma}}{1-\gamma}-\frac{L(-\epsilon(\mathcal{T}) \mathcal{T}, W)^{1+\psi}}{1+\psi}, \\
U_{B}(\mathcal{T}, W, 1 / \epsilon) & =\frac{X_{B}(W, 1 / \epsilon)^{1-\gamma}}{1-\gamma}-\frac{L(\mathcal{T}, W)^{1+\psi}}{1+\psi}
\end{aligned}
$$

where labor supply is given by $L(T, W)=\rho(1-T) / W$ and consumption is given by:

$$
X_{G}(W, \epsilon)=\xi \mu^{-1} \frac{1}{\frac{W}{\rho} A B(\varepsilon)}, \quad \text { and } \quad \tilde{X}_{B}(W, \epsilon)=\xi \mu^{-1} \frac{1}{\frac{W}{\rho} A \frac{B(\epsilon)}{\epsilon}}=\epsilon X_{G}(W, \epsilon),
$$

where $A=\left(a_{G}^{1-\sigma}+a_{B}^{1-\sigma}\right)^{\frac{1}{1-\sigma}}$ and $B(\epsilon):=B_{G}(\epsilon)=\epsilon B_{B}(1 / \epsilon)=\left(\frac{1}{2} b_{G}+\frac{1}{2} b_{B} \varepsilon^{1-\sigma}\right)^{\frac{1}{1-\sigma}}$. Differentiating the exchange rate condition and evaluating at $\mathcal{T}=0$ yields:

$$
\frac{\epsilon^{\prime}(0)}{\epsilon_{0}}=\frac{\left(1+\epsilon_{0}\right)}{\sigma}
$$


Differentiating $U_{G}$ and $U_{B}$ and evaluating at $\mathcal{T}=0$ gives

$$
\begin{aligned}
\frac{\partial U_{G}\left(0, W_{0}, \epsilon_{0}\right)}{\partial T} & =\frac{\partial U_{B}\left(0, W_{0}, 1 / \epsilon_{0}\right)}{\partial T}=\left(\frac{W_{0}}{\rho}\right)^{-(1+\psi)}, \\
W_{0} \frac{\partial U_{G}\left(0, W_{0}, \epsilon_{0}\right)}{\partial W} & =\left(\frac{W_{0}}{\rho}\right)^{-(1+\psi)}\left(-\kappa_{2} \frac{B\left(\varepsilon_{0}\right)^{\gamma-1}}{\frac{1}{2} B\left(\varepsilon_{0}\right)^{\gamma-1}+\frac{1}{2}\left(\frac{B\left(\varepsilon_{0}\right)}{\varepsilon_{0}}\right)^{\gamma-1}+1}\right), \\
W_{0} \frac{\partial U_{B}\left(0, W_{0}, 1 / \epsilon_{0}\right)}{\partial W} & =\left(\frac{W_{0}}{\rho}\right)^{-(1+\psi)}\left(\begin{array}{c}
\left.-\kappa_{2} \frac{B\left(\varepsilon_{0}\right)}{\varepsilon_{0}}\right)^{\gamma-1} \\
\frac{1}{2} B\left(\varepsilon_{0}\right)^{\gamma-1}+\frac{1}{2}\left(\frac{B\left(\varepsilon_{0}\right)}{\varepsilon_{0}}\right)^{\gamma-1}+1
\end{array}\right), \\
\frac{\partial U_{G}\left(0, W_{0}, \epsilon_{0}\right)}{\partial \varepsilon} \epsilon^{\prime}(0) & =-\left(\frac{W_{0}}{\rho}\right)^{-(1+\psi)}\left(\frac{\kappa_{2} \epsilon_{0}}{\sigma}\right)\left(\frac{B\left(\epsilon_{0}\right)^{\gamma-1}}{E B\left(\epsilon_{0}\right)}\right), \\
\frac{\partial \tilde{U}_{B}\left(0, W_{0}, \epsilon_{0}\right)}{\partial \epsilon} \epsilon^{\prime}(0) & =\left(\frac{W_{0}}{\rho}\right)^{-(1+\psi)}\left(\frac{\kappa_{2}}{\sigma}\right)\left(\frac{\left(\frac{B\left(\epsilon_{0}\right)}{\epsilon_{0}}\right)^{\gamma-1}}{E B_{\gamma}\left(\epsilon_{0}\right)}\right)
\end{aligned}
$$

where

$$
E B_{\gamma}\left(\epsilon_{0}\right)=\left(\frac{1}{2} B\left(\epsilon_{0}\right)^{\gamma-1}+\frac{1}{2}\left(\frac{B\left(\epsilon_{0}\right)}{\epsilon_{0}}\right)^{\gamma-1}\right) .
$$

Next, we differentiate the wage equation (17) and evaluate it at $\mathcal{T}=0$. Using equation (D.6), we compute

$$
(\gamma+\psi) \frac{W^{\prime}(0)}{W_{0}}=\frac{(1+\psi)}{2}\left(\epsilon_{0}-1\right)-\left(\frac{1}{2} \frac{B\left(\epsilon_{0}\right)^{\gamma-1} \epsilon_{0}\left(1+\frac{\gamma-1}{\sigma}\right)}{E B_{\gamma}\left(\varepsilon_{0}\right)}-\frac{1}{2} \frac{\left(\frac{B\left(\epsilon_{0}\right)}{\epsilon_{0}}\right)^{\gamma-1}\left(1+\frac{\gamma-1}{\sigma}\right)}{E B_{\gamma}\left(\epsilon_{0}\right)}\right) .
$$

\section{Small shocks and derivation of equation (28)}

Taking an approximation about $z=1$ so that

$\frac{1}{\underline{\delta}^{f}} \approx 1-\frac{1}{2}\left(\frac{(\gamma+\psi)\left(\gamma+\mu(\sigma-1)\left(\frac{\theta-1}{\theta}\right)\right)-\left((\gamma-1)\left(1+\frac{\gamma}{\sigma}\right)-\psi\right)\left(\sigma-\mu(\sigma-1)\left(\frac{\theta-1}{\theta}\right)\right)}{(\gamma+\psi)\left(1+\mu(\sigma-1)\left(\frac{\theta-1}{\theta}\right)\right)}\right)\left(\frac{\sigma-1}{\sigma}\right)(z-1)$.

Using $\underline{\lambda}:=(\underline{\delta}-1) / \underline{\delta}$ and the approximation $\underline{\delta} \approx 1+\underline{\lambda}$, and setting $\mu=\psi=(\theta-1) / \theta=1$, gives $(28)$ in the text.

\section{Proof of Proposition 3}

It has already been shown that for small shocks $\bar{\delta}^{c}<1$ and consequently $\bar{\delta}^{c}$ is positively related to $z$ for $z$ close to one: that is, transfers are more likely to be sustained for larger shocks close to $z=1$.

Equation (28) gives the approximation for $\underline{\delta}^{f}$ for $\mu=\psi=(\theta-1) / \theta=1$. It is clear that since $\sigma>1$, the bracketed term is strictly positive. Hence, we can conclude that by continuity $\underline{\delta}^{f}<1$ for $\mu$ and $\psi$ close to one and for $\theta$ large. Again, it follows that for small shocks $\bar{\delta}^{f}$ is positively related to $z$ for $z$ close to one.

Next, we show $\bar{\delta}^{c}>\underline{\delta}^{c}$ for $\mu$ close to one and for $\sigma>\psi^{-1}$. Note that as $\mu \rightarrow 1$, we get from above that

$$
\frac{1}{\underline{\delta}^{c}} \approx 1-\frac{1}{2}\left(\frac{\psi}{(\gamma+\psi)}+\frac{\gamma}{(\gamma+\psi) \kappa_{2}}\right) \gamma(\sigma-1)(z-1) .
$$


Comparing $\bar{\delta}^{c}$ with $\underline{\delta}^{c}$, for $\mu \rightarrow 1$, we have $\bar{\delta}^{c}>\underline{\delta}^{c}$ provided

$$
\frac{1}{2}\left(\frac{\psi}{(\gamma+\psi)}+\frac{\gamma}{(\gamma+\psi) \kappa_{2}}\right)>\frac{1}{4}\left(1+\frac{1}{\kappa_{1}}\right)\left(\frac{(1+\psi)}{(\gamma+\psi)}\right) .
$$

With $\kappa_{1}=\kappa_{2}(1+\psi) /(\gamma-1)$, this reduces to

$$
\psi>1-\frac{1+\gamma}{\kappa_{2}} .
$$

Since $\kappa_{2}=(\theta /(\theta-1))(\sigma /(\sigma-1))$, we have $\bar{\delta}^{c}>\underline{\delta}^{c}$ provided

$$
\psi>\frac{1}{\sigma}-\frac{(\sigma-1)}{\sigma}\left((1+\gamma) \frac{(\theta-1)}{\theta}-1\right) .
$$

Since the second term is positive and given continuity in $\mu$, we conclude that $\sigma>\psi^{-1}$ is sufficient for $\bar{\delta}^{c}>\underline{\delta}^{c}$ with $\mu$ close to one.

Comparing equations (26) and (28), it can be seen that for $\mu=\psi=(\theta-1) / \theta=1, \bar{\delta}^{f}>\underline{\delta}^{f}$ provided

$$
2\left(\gamma^{2}(\sigma-1)+\gamma\left(\sigma+(\sigma-1)^{2}\right)+\sigma(1+\sigma)\right)>(\gamma-\sigma(\sigma-1))((1+\sigma)+\gamma(\sigma-1)),
$$

or rewriting, provided

$$
\gamma^{2}(\sigma-1)+\gamma\left(\sigma^{3}-2 \sigma+1\right)+\sigma(1+\sigma)^{2}>0 .
$$

Since $\sigma>1$, the above inequality holds strictly and we can conclude that by continuity $\bar{\delta}^{f}>\underline{\delta}^{f}$ for $\mu$ and $\psi$ close to one and $\theta$ large.

Finally, we show that a sufficient condition for $\underline{\delta}^{f}>\underline{\delta}^{c}$ is $\sigma>1+\theta /(\theta-1)$. Write the approximations given above (taking $\mu=1$ ) as

$$
\begin{aligned}
\underline{\delta}^{c} & \approx 1+\frac{1}{2} \nu^{c}\left(\frac{\sigma-1}{\sigma}\right)(z-1), \\
\underline{\delta}^{f} & \approx 1+\frac{1}{2} \nu^{f}\left(\frac{\sigma-1}{\sigma}\right)(z-1),
\end{aligned}
$$

where

$$
\begin{aligned}
\nu^{c} & =\gamma \frac{\psi \sigma+\gamma(\sigma-1)\left(\frac{\theta-1}{\theta}\right)}{(\gamma+\psi)}, \\
\nu^{f} & =\frac{(\gamma+\psi)\left(\gamma(\sigma-1)\left(\frac{\theta-1}{\theta}\right)\right)-\left((\gamma-1)\left(1+\frac{\gamma}{\sigma}\right)-\psi\right)\left(\sigma-(\sigma-1)\left(\frac{\theta-1}{\theta}\right)\right)}{(\gamma+\psi)\left(1+(\sigma-1)\left(\frac{\theta-1}{\theta}\right)\right)} .
\end{aligned}
$$

Since $z<1$, we have $\underline{\delta}^{f}>\underline{\delta}^{c}$ when $\nu^{c}-\nu^{f}>0$. It can be shown that $\nu^{c}-\nu^{f}$ is increasing in $\gamma$, and thus, if $\nu^{c}-\nu^{f}>0$ for $\gamma=1$, then $\nu^{c}-\nu^{f}>0$ for all $\gamma>1$. Evaluating $\nu^{c}-\nu^{f}$ at $\gamma=1$ gives the following quadratic in $\sigma:^{38}$

$$
\frac{\theta-1}{\theta}\left(\frac{\theta-1}{\theta}+\psi\right) \sigma^{2}-\frac{\theta-1}{\theta}\left(2 \frac{\theta-1}{\theta}+\psi\right) \sigma-1-\psi+\left(\frac{\theta-1}{\theta}\right)^{2} .
$$

This is a convex quadratic with a positive determinant and one negative and one positive real root. Thus, a sufficient condition for $\nu^{c}-\nu^{f}>0$ is that $\sigma$ is larger than the upper root. It can be checked that the upper root is decreasing in $\psi$. Thus, a sufficient condition is that $\sigma$ is larger than the upper root as $\psi \rightarrow 0$. Calculating this limit gives $\sigma>1+(\theta /(\theta-1))$.

\footnotetext{
${ }^{38}$ Note that for $\psi=(\theta-1) / \theta=1$ this quadratic is $2 \sigma^{2}-3 \sigma-1$ as given in the main text.
} 


\section{Proof of Proposition 4}

The sign of the difference between $\bar{\delta}^{c}$ and $\underline{\delta}^{f}$ is determined by a quadratic in $\gamma$. With $\mu=1$ the coefficients of this quadratic are:

$$
\begin{aligned}
a(\sigma ; \theta) & =(\sigma-1)\left(\frac{\theta-1}{\theta}\right)\left(\sigma-2+\sigma(\sigma-1)\left(\frac{\theta-1}{\theta}\right)\right), \\
b(\sigma ; \theta, \psi) & =(\sigma-1)\left(\left(\frac{\theta-1}{\theta}\right) \sigma^{2}+2+\frac{3 \sigma}{\theta}\right)+\left(\frac{\theta}{\theta-1}\right)\left(\frac{1}{\theta}+\frac{(\psi-1) \sigma}{(\sigma-1)}\right) a(\sigma, \theta), \\
c(\sigma ; \theta, \psi) & =-2 \sigma\left(\sigma(1+\psi)-(\sigma-1)\left(\frac{\theta-1}{\theta}\right)\right) .
\end{aligned}
$$

For $\psi=(\theta-1) / \theta=1$, the quadratic in $\gamma$ determined by these coefficients is given by the LHS of inequality (29) in the text:

$$
a(\sigma)=(\sigma-1)\left(\sigma^{2}-2\right) ; \quad b(\sigma)=(\sigma-1)\left(\sigma^{2}+2\right) ; \quad c(\sigma)=-2 \sigma(1+\sigma) .
$$

Let $\Delta(\sigma)$ denote the discriminant of the quadratic as a function of $\sigma$ and let $\gamma^{u}(\sigma)$ and $\gamma^{l}(\sigma)$ denote the upper and lower real roots when the discriminant in non-negative. Likewise, let $\gamma^{*}(\sigma)=-b(\sigma ; \theta, \psi) /(2 a(\sigma ; \theta))$ denote the value of $\gamma$ at the turning point and let $v^{*}(\sigma)=-\left(\left(b(\sigma)^{2}\right) / 4 a(\sigma)\right)+c(\sigma)$ denote the value of the quadratic at the turning point. The roots satisfy $\frac{1}{2}\left(\gamma^{u}(\sigma)+\gamma^{l}(\sigma)\right)=\gamma^{*}(\sigma)$ and $\gamma^{u}(\sigma) \cdot \gamma^{l}(\sigma)=$ $c(\sigma ; \theta, \psi) / a(\sigma ; \theta)$.

First let

$$
\bar{\sigma}(\theta):=\frac{-1+\frac{\theta-1}{\theta}+\sqrt{1+6 \frac{\theta-1}{\theta}+\left(\frac{\theta-1}{\theta}\right)^{2}}}{2 \frac{\theta-1}{\theta}} .
$$

We have $\bar{\sigma}(\theta)$ decreasing and

$$
\lim _{\theta \rightarrow 1} \bar{\sigma}(\theta)=2 ; \quad \lim _{\theta \rightarrow \infty} \bar{\sigma}(\theta)=\sqrt{2} .
$$

For $\sigma<\bar{\sigma}(\theta), a(\sigma, \theta)<0$ and for $\sigma>\bar{\sigma}(\theta), a(\sigma, \theta)>0$. We have $a(\bar{\sigma}(\theta), \theta)=0$ and $\bar{\sigma}(\theta) \in(\sqrt{2}, 2)$.

It can be shown that

$$
\lim _{\sigma \downarrow \bar{\sigma}(\theta)} \gamma^{u}(\sigma)=-\frac{c(\bar{\sigma}(\theta))}{b(\bar{\sigma}(\theta))}=(1+\psi)+(1+2 \psi) h(\theta)
$$

where

$$
h(\theta)=\frac{\left(\frac{\theta-1}{\theta}\right)\left(3\left(1-\frac{\theta-1}{\theta}\right)-\sqrt{1+6 \frac{\theta-1}{\theta}+\left(\frac{\theta-1}{\theta}\right)^{2}}\right)}{4\left(1-\frac{\theta-1}{\theta}\left(3-\frac{\theta-1}{\theta}\right)\right)} .
$$

It is readily checked that $h(1)=0, h(\theta)$ is increasing and $\lim _{\theta \rightarrow \infty} h(\theta)=\sqrt{2} / 2$. With $\psi>0$ and $\theta>1$, we have $\lim _{\sigma \downarrow \bar{\sigma}(\theta)} \gamma^{u}(\sigma)>1$. Also

$$
\lim _{\sigma \rightarrow \infty} \gamma^{u}(\sigma)=0
$$

We will show that $\gamma^{u}(\sigma)$ crosses the $\gamma=1$ line just once, that is, there is a unique value of $\hat{\sigma}$ such that $\gamma^{u}(\hat{\sigma})=1, \gamma^{u}(\sigma)>1$ for all $\sigma \in\left(\bar{\sigma}(\theta), \hat{\sigma}\right.$ and $\gamma^{u}(\sigma)<1$ for all $\sigma>\hat{\sigma}$. We have that $\gamma^{u}(\sigma)=1$ implies $a(\sigma)+b(\sigma)+c(\sigma)=0$. The solution to this equation is the solution to a quadratic in $\sigma$ :

$$
\alpha(\psi, \theta) \sigma^{2}+\beta(\psi, \theta) \sigma+\chi(\psi, \theta)=0
$$


where

$$
\alpha(\psi, \theta)=\frac{\theta-1}{\theta}(1+\psi) ; \quad \beta(\psi, \theta)=1-\psi-3 \frac{\theta-1}{\theta}-\psi \frac{\theta-1}{\theta} ; \quad \chi(\psi, \theta)=-2\left(1+\psi-\frac{\theta-1}{\theta}\right) .
$$

It is clear that $\alpha(\psi, \theta)>0$ and $\chi(\psi, \theta)<0$, so the discriminant of this quadratic is positive and there are two real roots (one positive, one negative). Let $\sigma^{u}(\psi, \theta)$ denote the upper root. It can be checked that $\sigma^{u}(\psi, \theta)>\bar{\sigma}(\theta)$ for all $\psi>0$ and $\theta>1$. It can also be checked that $\sigma^{u}(\psi, \theta)$ is increasing in $\psi$ and decreasing in $\theta$. Also,

$$
\lim _{\psi \rightarrow \infty} \sigma^{u}(\psi, \theta)=\frac{1+\frac{\theta-1}{\theta}+\sqrt{1+10 \frac{\theta-1}{\theta}+\left(\frac{\theta-1}{\theta}\right)^{2}}}{2 \frac{\theta-1}{\theta}},
$$

and

$$
\lim _{\theta \rightarrow \infty} \sigma^{u}(\psi, \theta)=1+\sqrt{\frac{1+3 \psi}{1+\psi}} .
$$

Thus, for $\theta$ large and $\mu$ close to one, $\sigma>1+\sqrt{(1+\psi)(1+3 \psi)} /(1+\psi)$ is a sufficient condition for $\underline{\delta}^{f}>\bar{\delta}^{c}$. It can be seen that for $\psi=1, \lim _{\theta \rightarrow \infty} \sigma^{u}(\psi, 1)=1+\sqrt{2}$ and therefore, for $\mu=\psi=(\theta-1) / \theta=1$, a sufficient condition for (29) to hold for any $\gamma>1$ is $\sigma>1+\sqrt{2}$. Similarly, (30) holds for $\gamma>\sqrt{2}(1+\sqrt{2})$. Since $\sigma^{u}(\psi, \theta)$ is increasing in $\psi$, a sufficient condition for $\underline{\delta}^{f}>\bar{\delta}^{c}$ can be obtained for any $\psi$ and $\theta$ large by taking the limit:

$$
\lim _{(\theta \rightarrow \infty, \psi \rightarrow \infty)} \sigma^{u}(\psi, \theta)=1+\sqrt{3}
$$

\section{Appendix E Transaction costs}

In the presence of iceberg exchange rate transaction costs, prices of exported goods are multiplied by $\tau$ so that the Home price of Foreign goods is $\varepsilon \tau p^{*}\left(v^{*}\right)$ and the Foreign price of Home goods is $(1 / \varepsilon) \tau p(v)$. The price indices depend on transaction costs such that $P^{1-\sigma}=\int_{0}^{1} p(v)^{1-\sigma} d v+\int_{0}^{1}\left(\varepsilon \tau p^{*}\left(v^{*}\right)\right)^{1-\sigma} d v^{*}$ and $\left(P^{*}\right)^{1-\sigma}=\int_{0}^{1}\left(p^{*}\left(v^{*}\right)\right)^{1-\sigma} d v^{*}+\int_{0}^{1}(\tau p(v) / \varepsilon)^{1-\sigma} d v$. The demands for the variety $v$ of a Home firm must here be split between the demand by domestic and foreign consumers

$$
\mu \frac{(p(v))^{-\sigma}}{P^{1-\sigma}}\left(Y+T+M_{0}\right) \quad \text { and } \tau\left(\mu \frac{(\tau p(v) / \varepsilon)^{-\sigma}}{\left(P^{*}\right)^{1-\sigma}}\left(Y^{*}+T^{*}+M_{0}^{*}\right)\right) .
$$

In the second expression, the term $\tau$ in front of the parentheses accounts for the fact that the firms must produce $\tau$ times the actual consumption and the term in $\tau$ within the parentheses accounts for the fact that the consumers pays $\tau$ times her actual consumption. We assume that governments' transfers are not subject to transaction cost so that $T=-\varepsilon T^{*}$.

The Home firm chooses the same input mix as before so that it faces a wage $W$. Under monopolistic competition the firm takes as aggregate variable as givens and therefore faces iso-elastic demand functions. It thus sets its price with constant markup on costs: $p(v)=p:=a W / \rho$. Similarly, for Foreign firms, $p^{*}\left(v^{*}\right)=p^{*}:=a^{*} W^{*} / \rho$. The demand is the same for all producers in the same country $(D:=D(v)$ and 
$\left.D^{*}:=D^{*}\left(v^{*}\right)\right)$ and it is equal to the sum of the two previous expressions:

$$
\begin{aligned}
D & =\mu\left(\frac{a W}{\rho}\right)^{-\sigma}\left(\frac{\left(Y+T+M_{0}\right)}{P^{1-\sigma}}+\tau\left(\frac{\tau}{\varepsilon}\right)^{-\sigma} \frac{\left(Y^{*}+T^{*}+M_{0}^{*}\right)}{\left(P^{*}\right)^{1-\sigma}}\right), \\
D^{*} & =\mu\left(\frac{a^{*} W^{*}}{\rho}\right)^{-\sigma}\left(\frac{\left(Y^{*}+T^{*}+M_{0}^{*}\right)}{\left(P^{*}\right)^{1-\sigma}}+\tau(\tau \varepsilon)^{-\sigma} \frac{\left(Y+T+M_{0}\right)}{P^{1-\sigma}}\right),
\end{aligned}
$$

where

$$
\begin{gathered}
P=(W / \rho)\left((a)^{1-\sigma}+\left(\tau \frac{\varepsilon W^{*}}{W}\right)^{1-\sigma}\left(a^{*}\right)^{1-\sigma}\right)^{\frac{1}{1-\sigma}}, \\
P^{*}=\left(W^{*} / \rho\right)\left(\left(a^{*}\right)^{1-\sigma}+\left(\tau \frac{W}{\varepsilon W^{*}}\right)^{1-\sigma}(a)^{1-\sigma}\right)^{\frac{1}{1-\sigma}} .
\end{gathered}
$$

In contrast to the model without transaction cost, we here have $P \neq \varepsilon P^{*}$. We write the price indices as

$$
P(W, \varepsilon)=(W / \rho) A B(\varepsilon), \quad \text { and } \quad P^{*}(W, \varepsilon)=\left(W^{*} / \rho\right) A B^{*}(\varepsilon),
$$

where

$$
B(\varepsilon):=\left(\frac{1}{2} b+\frac{1}{2} b^{*}\left(\tau \frac{\varepsilon W^{*}}{W}\right)^{1-\sigma}\right)^{\frac{1}{1-\sigma}}, \quad \text { and } \quad B^{*}(\varepsilon):=\left(\frac{1}{2} b^{*}+\frac{1}{2} b\left(\tau \frac{W}{\varepsilon W^{*}}\right)^{1-\sigma}\right)^{\frac{1}{1-\sigma}}
$$

Given the symmetry of countries and states of nature, we have $W=W^{*}$. It can be shown that $W$ is given by expression (A.8) with the above $B(\varepsilon)$ and $B^{*}(\varepsilon)$.

We now focus on the flexible exchange rate regime. The money supply equalizes the money demand so that $Y+T=1$ as before. Because $Y=W L / \rho$, the labor supply is equal to $L=\rho Y / W=\rho(1-T) / W$ at Home and $L^{*}=\rho\left(1-T^{*}\right) / W^{*}$ abroad. The labor supply is equal its demand at Home and abroad so that $L^{*}=a^{*} D^{*}$. This yields $L / L^{*}=\left(a / a^{*}\right)\left(D / D^{*}\right)$. The exchange rate is then be computed as the solution of

$$
\frac{1-T}{1+\frac{T}{\varepsilon}}\left(\frac{a^{*}}{a}\right)^{1-\sigma}=\varepsilon^{\sigma}\left(\frac{1+\tau^{1-\sigma} \varepsilon\left(\frac{P}{\varepsilon P^{*}}\right)^{1-\sigma}}{\tau^{1-\sigma}+\varepsilon\left(\frac{P}{\varepsilon P^{*}}\right)^{1-\sigma}}\right) .
$$

We can further write this as

$$
\frac{1-T}{1+\frac{T}{\varepsilon}}=\frac{b}{b^{*}} \varepsilon^{\sigma}\left(\frac{1+\tau^{1-\sigma} \varepsilon \Upsilon(\varepsilon, \tau)}{\tau^{1-\sigma}+\varepsilon \Upsilon(\varepsilon, \tau)}\right)
$$

where

$$
\Upsilon(\varepsilon, \tau) \equiv\left(\frac{P}{\varepsilon P^{*}}\right)^{1-\sigma}=\frac{b+b^{*} \tau^{1-\sigma} \varepsilon^{1-\sigma}}{b^{*} \varepsilon^{1-\sigma}+b \tau^{1-\sigma}} .
$$

Note that $\Upsilon^{\frac{1}{1-\sigma}}$ denotes the welfare-based real exchange rate. The terms in the parentheses of the RHS of (E.1) and (E.2) encapsulates the effect of transaction costs on the exchange rate. As expected, for $\tau=1$, we have that $\Upsilon(\varepsilon, 1)=1$, so that the exchange rate is determined by the expression (15) in the absence of transaction cost. It can be shown that $\lim _{\tau \rightarrow 1, b / b^{*} \rightarrow 1} \mathrm{~d} \varepsilon / \mathrm{d} \tau=0$. As a consequence, the exchange rate is almost invariant to very small transaction costs and productivity differences. For larger values, transaction 
costs create a home bias so that the terms of trade, $P / P^{*}=\varepsilon(\Upsilon(\varepsilon, \tau))^{\frac{1}{1-\sigma}}$, differs from the nominal exchange rates $\varepsilon$. Finally expected utilities are given by the same expression as (18) taking into account the new values for $B$ and $B^{*}$. Those expressions are used to compute the values in Figure 4.

We now consider the consumption sharing transfers. Since transaction costs affect the exchange rate, they will also alter the values of transfers that equalise consumption. As before, Home consumption in state $s$ is equal to $X_{s}=(\xi / \mu)\left(1 / P_{s}\right)$ and consumption sharing implies $P_{s}=P_{s}^{*}$ and therefore $B_{s}=B_{s}^{*}$, which implies that $\Upsilon(\varepsilon, \tau)=\varepsilon^{\sigma-1}$. Since $P_{s} \neq \varepsilon_{s} P_{s}^{*}$, consumption sharing does not lead to the unit exchange rate $\varepsilon_{s}=1$. Solving $B_{s}=B_{s}^{*}$ for $\varepsilon_{s}$ yields

$$
\varepsilon_{s}^{1-\sigma}=\frac{1}{2 \tau^{1-\sigma}}\left(\left(1-\frac{b_{s}}{b_{s}^{*}}\right)+\sqrt{\left(1-\frac{b_{s}}{b_{s}^{*}}\right)^{2}+4 \tau^{2(1-\sigma)}\left(\frac{b_{s}}{b_{s}^{*}}\right)}\right) .
$$

The consumption sharing transfer is deduced from the exchange rate expression (E.2) as:

$$
T_{s}=\frac{1-\frac{b_{s}}{b_{s}^{*}} \Gamma_{s}}{1+\frac{1}{\varepsilon_{s}} \frac{b_{s}}{b_{s}^{*}} \Gamma_{s}}, \quad \text { where } \quad \Gamma_{s}=\varepsilon_{s}^{\sigma} \tau^{\sigma-1} \frac{1+\tau^{1-\sigma} \varepsilon_{s}^{\sigma}}{1+\tau^{\sigma-1} \varepsilon_{s}^{\sigma}} .
$$

As expected, for $\tau=1$, we get $\varepsilon_{s}=1, \Gamma_{s}=1$ and $T_{s}=\frac{b_{s}^{*}-b_{s}}{b_{s}^{*}+b_{s}}=\frac{1}{2}\left(b_{s}^{*}-b_{s}\right)$. The computation of the critical discount factor $\underline{\delta}^{f}$ and $\bar{\delta}^{f}$ follows the same procedure as with no transaction costs. 


\section{Appendix S Supplementary - Not for Publication}

This supplementary Appendix provides details of the derivations given in Appendix D.

\section{Currency area}

Recall that $\epsilon=1$ and therefore that the expected discounted utility in state $G$ is $V_{G}(\mathcal{T}):=\tilde{V}_{G}(\mathcal{T}, W(\mathcal{T}))$ where

$$
\begin{aligned}
\tilde{V}_{G}(\mathcal{T}, W(\mathcal{T})):= & U_{G}(-\mathcal{T}, W(\mathcal{T}))-U_{G}(0, W(0))+U_{G}(0, W(0))-U_{G}(0, W(\mathcal{T})) \\
& \quad+\frac{1}{(1-\delta)}\left(\left(\frac{\delta}{2}\right)\left(U_{G}(-\mathcal{T}, W(\mathcal{T}))-U_{G}(0, W(0))\right)+\left(\frac{\delta}{2}\right)\left(U_{B}(\mathcal{T}, W(\mathcal{T}))-U_{B}(0, W(0))\right)\right) \\
= & U_{G}(0, W(0))-U_{G}(0, W(\mathcal{T})) \\
& \quad+\frac{1}{(1-\delta)}\left(\left(1-\frac{\delta}{2}\right)\left(U_{G}(-\mathcal{T}, W(\mathcal{T}))-U_{G}(0, W(0))\right)+\left(\frac{\delta}{2}\right)\left(U_{B}(\mathcal{T}, W(\mathcal{T}))-U_{B}(0, W(0))\right)\right)
\end{aligned}
$$

We have

$$
\begin{aligned}
V_{G}^{\prime}(\mathcal{T})=\frac{\partial \tilde{V}_{G}(\mathcal{T}, W(\mathcal{T}))}{\partial \mathcal{T}}+\frac{\partial \tilde{V}_{G}(\mathcal{T}, W(\mathcal{T}))}{\partial W} W^{\prime}(\mathcal{T}) \\
=\left(\frac{1}{1-\delta}\right)\left(\left(\frac{\delta}{2}\right) \frac{\partial U_{B}(\mathcal{T}, W(\mathcal{T}))}{\partial T}-\left(1-\frac{\delta}{2}\right) \frac{\partial U_{G}(-\mathcal{T}, W(\mathcal{T}))}{\partial T}\right) \\
\quad-\frac{W^{\prime}(\mathcal{T})}{W(\mathcal{T})} \frac{\partial U_{G}(0, W(\mathcal{T}))}{\partial W} W(\mathcal{T}) \\
\quad+\left(\frac{1}{1-\delta}\right) \frac{W^{\prime}(\mathcal{T})}{W(\mathcal{T})}\left(\left(1-\frac{\delta}{2}\right) \frac{\partial U_{G}(-\mathcal{T}, W(\mathcal{T}))}{\partial W} W(\mathcal{T})+\left(\frac{\delta}{2}\right) \frac{\partial U_{B}(\mathcal{T}, W(\mathcal{T}))}{\partial W} W(\mathcal{T})\right)
\end{aligned}
$$

Evaluating at $\mathcal{T}=0$, we get

$$
\begin{aligned}
V_{G}^{\prime}(0)= & \left(\frac{1}{1-\delta}\right)\left(\frac{W^{\prime}(0)}{W(0)}\right)\left(\frac{\delta}{2}\right)\left(\frac{\partial U_{G}(0, W(0))}{\partial W} W(0)+\frac{\partial U_{B}(0, W(0))}{\partial W} W(0)\right) \\
& +\left(\frac{1}{1-\delta}\right)\left(\left(\frac{\delta}{2}\right) \frac{\partial U_{B}(0, W(0))}{\partial T}-\left(1-\frac{\delta}{2}\right) \frac{\partial U_{G}(0, W(0))}{\partial T}\right) \\
= & \left(\frac{1}{1-\delta}\right)\left(\delta\left\{\mathrm{E}\left[\frac{\partial U_{s}(0, W(0))}{\partial T}\right]+\left(\frac{W^{\prime}(0)}{W(0)}\right) \mathrm{E}\left[\frac{\partial U_{s}(0, W(0))}{\partial W} W(0)\right]\right\}-\frac{\partial U_{G}(0, W(0))}{\partial T}\right) .
\end{aligned}
$$

We can therefore rewrite the requirement that $V_{G}^{\prime}(0)>0$ as $\delta>\underline{\delta}$ where

$$
\frac{1}{\underline{\delta}}=\frac{\left(\mathrm{E}\left[\frac{\partial U_{s}(0, W(0))}{\partial T}\right]+\left(\frac{W^{\prime}(0)}{W(0)}\right) \mathrm{E}\left[\frac{\partial U_{s}(0, W(0))}{\partial W} W(0)\right]\right)}{\frac{\partial U_{G}(0, W(0))}{\partial T}} .
$$

This is equation (D.1) in Appendix D. We now consider each of the terms in (S.1). First, differentiating 
the wage equation (A.3) with $T_{G}=-\mathcal{T}$ and $T_{B}=\mathcal{T}$ and recalling that $A_{s}=A$

$$
\begin{aligned}
\frac{W^{\prime}(\mathcal{T})}{W(\mathcal{T})} & =\frac{\mathrm{d}}{\mathrm{d} \mathcal{T}} \ln W(\mathcal{T}) \\
& =\frac{\mathrm{d}}{\mathrm{d} \mathcal{T}} \ln \left(\kappa_{0} \frac{\mathrm{E}\left[b_{s}^{1+\psi}\right]}{\mathrm{E}\left[\left(\mu\left(b_{s}+T_{s}\right)+(1-\mu)\right)^{-\gamma} b_{s} A_{s}^{\gamma-1}\right]}\right)^{\frac{1}{\gamma+\psi}} \\
& =-\frac{1}{\gamma+\psi} \frac{\mathrm{d}}{\mathrm{d} \mathcal{T}} \ln \left(\mathrm{E}\left[\left(\mu\left(b_{s}+T_{s}\right)+(1-\mu)\right)^{-\gamma} b_{s} A_{s}^{\gamma-1}\right]\right) \\
& =-\frac{1}{\gamma+\psi} \frac{\frac{\mathrm{d}}{\mathrm{d} \mathcal{T}}\left(\mathrm{E}\left[\left(\mu\left(b_{s}+T_{s}\right)+(1-\mu)\right)^{-\gamma} b_{s} A_{s}^{\gamma-1}\right]\right)}{\mathrm{E}\left[\left(\mu\left(b_{s}+T_{s}\right)+(1-\mu)\right)^{-\gamma} b_{s} A_{s}^{\gamma-1}\right]} \\
& =-\frac{1}{\gamma+\psi} \frac{\left(\mathrm{E}\left[\frac{\mathrm{d}}{\mathrm{d} \mathcal{T}}\left(\mu\left(b_{s}+T_{s}\right)+(1-\mu)\right)^{-\gamma} b_{s}\right]\right)}{\mathrm{E}\left[\left(\mu\left(b_{s}+T_{s}\right)+(1-\mu)\right)^{-\gamma} b_{s}\right]} \\
& =\frac{\mu \gamma}{\gamma+\psi} \frac{\left(\mathrm{E}\left[\left(\mu\left(b_{s}+T_{s}\right)+(1-\mu)\right)^{-\gamma-1} \frac{\mathrm{d} T_{s}}{\mathrm{~d} \mathcal{T}} b_{s}\right]\right)}{\mathrm{E}\left[\left(\mu\left(b_{s}+T_{s}\right)+(1-\mu)\right)^{-\gamma} b_{s}\right]}
\end{aligned}
$$

which can be rewritten as

$$
\frac{W^{\prime}(\mathcal{T})}{W(\mathcal{T})}=-\frac{\mu \gamma}{\gamma+\psi} \frac{\left(\left(\mu\left(b_{G}-\mathcal{T}\right)+(1-\mu)\right)^{-\gamma-1} b_{G}-\left(\mu\left(b_{B}+\mathcal{T}\right)+(1-\mu)\right)^{-\gamma-1} b_{B}\right)}{\left(\left(\mu\left(b_{G}-\mathcal{T}\right)+(1-\mu)\right)^{-\gamma} b_{G}+\left(\mu\left(b_{B}+\mathcal{T}\right)+(1-\mu)\right)^{-\gamma} b_{B}\right)} .
$$

Next, recall that $L_{s}(W)=b_{s} \rho / W$ and with $M_{0}=(1-\mu) / \mu$ and $A_{s}=A, X_{s}\left(T_{s}, W\right)=\xi\left(\mu\left(b_{s}+T_{s}\right)+(1-\right.$ $\mu))(\rho /(\mu W A))$. Therefore

$$
W \frac{\partial X_{s}\left(T_{s}, W\right)}{\partial W}=-X_{s}\left(T_{s}, W\right) \text { and } \quad W \frac{\mathrm{d} L_{s}(W)}{\mathrm{d} W}=-L_{s}(W)
$$

and hence,

$$
\begin{aligned}
W \frac{\partial U_{s}\left(T_{s}, W\right)}{\partial W} & =W\left(X_{s}\left(T_{s}, W\right)^{-\gamma} \frac{\partial X_{s}\left(T_{s}, W\right)}{\partial W}-L_{s}(W)^{\psi} \frac{\mathrm{d} L_{s}(W)}{\mathrm{d} W}\right) \\
& =-\left(X_{s}\left(T_{s}, W\right)^{1-\gamma}-L_{s}(W)^{1+\psi}\right) .
\end{aligned}
$$

Then substituting for $X_{s}(T, W)$ and $L_{s}(W)$ using (A.4) gives

$$
W \frac{\partial U_{s}\left(T_{s}, W\right)}{\partial W}=-\left(\frac{W}{\rho}\right)^{-(1+\psi)} \kappa_{2} \mathrm{E}\left[b_{s}^{1+\psi}\right]\left(\frac{\left(\mu\left(b_{s}+T_{s}\right)+(1-\mu)\right)^{1-\gamma}}{\mathrm{E}\left[\left(\mu\left(b_{s}+T_{s}\right)+(1-\mu)\right)^{-\gamma} b_{s}\right]}-\frac{1}{\kappa_{2}} \frac{b_{s}^{1+\psi}}{\mathrm{E}\left[b_{s}^{1+\psi}\right]}\right)
$$

where $\kappa_{2}=\theta /((\theta-1) \mu \rho)$. Finally, using $\partial X_{s}(T, W) / \partial T=\mu X_{s}(T, W) /\left(\mu\left(b_{s}+T_{s}\right)+(1-\mu)\right)$ and using (A.4) gives

$$
\begin{aligned}
\frac{\partial U_{s}\left(T_{s}, W\right)}{\partial T} & =X_{s}\left(T_{s}, W\right)^{-\gamma} \frac{\partial X_{s}\left(T_{s}, W\right)}{\partial T} \\
& =\frac{\mu}{\left(\mu\left(b_{s}+T_{s}\right)+(1-\mu)\right)} X_{s}\left(T_{s}, W\right)^{1-\gamma}
\end{aligned}
$$


Again substituting for $X_{s}(T, W)$ and using (A.4) gives

$$
\frac{\partial U_{s}\left(T_{s}, W\right)}{\partial T}=\mu\left(\frac{W}{\rho}\right)^{-(1+\psi)} \kappa_{2} \mathrm{E}\left[b_{s}^{1+\psi}\right]\left(\frac{\left(\mu\left(b_{s}+T_{s}\right)+(1-\mu)\right)^{-\gamma}}{\mathrm{E}\left[\left(\mu\left(b_{s}+T_{s}\right)+(1-\mu)\right)^{-\gamma} b_{s}\right]}\right)
$$

Evaluating (S.2) at $\mathcal{T}=0$, and (S.3) and (S.4) at $T_{s}=0$, taking expectations and substituting into (S.1) gives

$$
\frac{1}{\underline{\delta}}=\frac{\mathrm{E}\left[\left(\mu b_{s}+(1-\mu)\right)^{-\gamma}\right]+\frac{\gamma}{\gamma+\psi}\left(\frac{\mathrm{E}\left[\left(\mu b_{s}+(1-\mu)\right)^{1-\gamma}\right]}{\mathrm{E}\left[\left(\mu b_{s}+(1-\mu)\right)^{-\gamma} b_{s}\right]}-\frac{1}{\kappa_{2}}\right)\left(\frac{1}{2}\left(\mu b_{G}+(1-\mu)\right)^{-\gamma-1}-\frac{1}{2}\left(\mu b_{B}+(1-\mu)\right)^{-\gamma-1}\right)}{\left(\mu b_{G}+(1-\mu)\right)^{-\gamma}} .
$$

Taking a first-order approximation of this equation about $z=1$ gives

$$
\frac{1}{\underline{\delta}^{c}} \approx 1-\frac{1}{2}\left(\frac{\mu \psi}{(\gamma+\psi)}+\frac{\mu \gamma+(1-\mu)\left(\kappa_{2}-1\right)}{(\gamma+\psi) \kappa_{2}}\right) \gamma(\sigma-1)(z-1) .
$$

Note the term in the bracket is positive because $\kappa_{2}>1$. (This is equivalent to positive gains from risksharing). Hence, we can conclude that $\underline{\delta}^{c}<1$ for small schocks. Substituting in for $\kappa_{2}$ gives

$$
\frac{1}{\underline{\delta}^{c}} \approx 1-\frac{1}{2}\left(\frac{1+\mu(\psi-1)}{(\gamma+\psi)}+\frac{\mu(\mu \gamma-(1-\mu))(\sigma-1)\left(\frac{\theta-1}{\theta}\right)}{(\gamma+\psi) \sigma}\right) \gamma(\sigma-1)(z-1)
$$

Using $\underline{\lambda}:=(\underline{\delta}-1) / \underline{\delta}$ and the approximation $\underline{\delta} \approx 1+\underline{\lambda}$ and setting $\mu=\psi=(\theta-1) / \theta=1$ gives $(27)$ in the text.

Note that as $\mu \rightarrow 1$, we get

$$
\frac{1}{\underline{\delta}^{c}} \approx 1-\frac{1}{2}\left(\frac{\psi}{(\gamma+\psi)}+\frac{\gamma}{(\gamma+\psi) \kappa_{2}}\right) \gamma(\sigma-1)(z-1) .
$$

Comparing $\bar{\delta}^{c}$ with $\underline{\delta}^{c}$, for $\mu=1$, we have $\bar{\delta}^{c}>\underline{\delta}^{c}$ provided

$$
\frac{1}{2}\left(\frac{\psi}{(\gamma+\psi)}+\frac{\gamma}{(\gamma+\psi) \kappa_{2}}\right)>\frac{1}{4}\left(1+\frac{1}{\kappa_{1}}\right)\left(\frac{(1+\psi)}{(\gamma+\psi)}\right)
$$

With $\kappa_{1}=\kappa_{2}(1+\psi) /(\gamma-1)$, this reduces to

$$
\psi>1-\frac{1+\gamma}{\kappa_{2}}
$$

Since $\kappa_{2}=(\theta /(\theta-1))(\sigma /(\sigma-1))$, we have $\bar{\delta}^{c}>\underline{\delta}^{c}$ provided

$$
\psi>\frac{1}{\sigma}-\frac{(\sigma-1)}{\sigma}\left((1+\gamma) \frac{(\theta-1)}{\theta}-1\right) .
$$

Since the second term is positive and given continuity in $\mu$, we conclude that $\psi>1 / \sigma$ is sufficient for $\bar{\delta}^{c}>\underline{\delta}^{c}$ with $\mu$ close to one. 


\section{Flexible case}

Let $V_{G}(\mathcal{T}):=\hat{V}_{G}(\mathcal{T}, W(\mathcal{T}), \epsilon(\mathcal{T}))$ denote expected discounted net utility in the good state. Writing $W_{0}:=$ $W(0)$ and $\epsilon_{0}:=\epsilon(0)$, where we have

$$
\begin{aligned}
\hat{V}_{G}(\mathcal{T}, W(\mathcal{T}), \epsilon(\mathcal{T})):= & U_{G}\left(0, W_{0}, \epsilon_{0}\right)-U_{G}\left(0, W(\mathcal{T}), \epsilon_{0}\right) \\
& +\frac{1}{(1-\delta)}\left(\left(1-\frac{\delta}{2}\right)\left(U_{G}(-\varepsilon(\mathcal{T}) \mathcal{T}, W(\mathcal{T}), \epsilon(\mathcal{T}))-U_{G}\left(0, W_{0}, \epsilon_{0}\right)\right)\right) \\
& +\frac{1}{(1-\delta)}\left(\left(\frac{\delta}{2}\right)\left(U_{B}(\mathcal{T}, W(\mathcal{T}), 1 / \epsilon(\mathcal{T}))-U_{B}\left(0, W_{0}, 1 / \epsilon_{0}\right)\right)\right)
\end{aligned}
$$

Differentiating

$$
V_{G}^{\prime}(\mathcal{T})=\frac{\partial \hat{V}_{G}(\mathcal{T}, W(\mathcal{T}), \epsilon(\mathcal{T}))}{\partial \mathcal{T}}+\frac{\partial \hat{V}_{G}(\mathcal{T}, W(\mathcal{T}), \epsilon(\mathcal{T}))}{\partial W} W^{\prime}(\mathcal{T})+\frac{\partial \hat{V}_{G}(\mathcal{T}, W(\mathcal{T}), \epsilon(\mathcal{T}))}{\partial \epsilon} \epsilon^{\prime}(\mathcal{T})
$$

where

$$
\begin{aligned}
\frac{\partial \hat{V}_{G}(\mathcal{T}, W(\mathcal{T}), \epsilon(\mathcal{T}))}{\partial \mathcal{T}}= & \left(\frac{1}{1-\delta}\right)\left(\left(\frac{\delta}{2}\right) \frac{\partial U_{B}(\mathcal{T}, W(\mathcal{T}), 1 / \epsilon(\mathcal{T}))}{\partial T}-\left(1-\frac{\delta}{2}\right) \frac{\partial U_{G}(-\epsilon(\mathcal{T}) \mathcal{T}, W(\mathcal{T}), \epsilon(\mathcal{T}))}{\partial T} \epsilon(\mathcal{T})\right) \\
\frac{\partial \hat{V}_{G}(\mathcal{T}, W(\mathcal{T}), \epsilon(\mathcal{T}))}{\partial W} W^{\prime}(\mathcal{T})= & \left(\frac{1}{1-\delta}\right) \frac{W^{\prime}(\mathcal{T})}{W(\mathcal{T})}\left(\frac{\delta}{2} \frac{\partial U_{G}(-\epsilon(\mathcal{T}) \mathcal{T}, W(\mathcal{T}), \epsilon(\mathcal{T}))}{\partial W} W(\mathcal{T})+\frac{\delta}{2} \frac{\partial U_{B}(\mathcal{T}, W(\mathcal{T}), 1 / \epsilon(\mathcal{T}))}{\partial W} W(\mathcal{T})\right) \\
\frac{\partial \hat{V}_{G}(\mathcal{T}, W(\mathcal{T}), \epsilon(\mathcal{T}))}{\partial \epsilon} \epsilon^{\prime}(\mathcal{T})= & \left(\frac{1}{1-\delta}\right)\left(\left(1-\frac{\delta}{2}\right) \frac{\partial U_{G}(-\epsilon(\mathcal{T}) \mathcal{T}, W(\mathcal{T}), \epsilon(\mathcal{T}))}{\partial \varepsilon} \epsilon^{\prime}(\mathcal{T})-\left(\frac{\delta}{2}\right) \frac{\partial U_{B}(\mathcal{T}, W(\mathcal{T}), 1 / \epsilon(\mathcal{T}))}{\partial \varepsilon}\left(\frac{\epsilon^{\prime}(\mathcal{T})}{\epsilon(\mathcal{T})^{2}}\right)\right) \\
& -\left(\frac{1}{1-\delta}\right)\left(\left(1-\frac{\delta}{2}\right) \frac{\partial U_{G}(-\epsilon(\mathcal{T}) \mathcal{T}, W(\mathcal{T}), \epsilon(\mathcal{T}))}{\partial T} \epsilon^{\prime}(\mathcal{T}) \mathcal{T}\right)
\end{aligned}
$$

Evaluating at $\mathcal{T}=0$ gives

$$
\begin{aligned}
\frac{\partial \hat{V}_{G}\left(0, W_{0}, \epsilon_{0}\right)}{\partial \mathcal{T}} & =\left(\frac{1}{1-\delta}\right)\left(\left(\frac{\delta}{2}\right) \frac{\partial U_{B}\left(0, W_{0}, 1 / \epsilon_{0}\right)}{\partial T}-\left(1-\frac{\delta}{2}\right) \frac{\partial U_{G}\left(0, W_{0}, \epsilon_{0}\right)}{\partial T} \epsilon_{0}\right) \\
\frac{\partial \hat{V}_{G}\left(0, W_{0}, \epsilon_{0}\right)}{\partial W} W^{\prime}(0) & =\left(\frac{1}{1-\delta}\right) \frac{W^{\prime}(0)}{W_{0}}\left(\frac{\delta}{2} \frac{\partial U_{G}\left(0, W_{0}, \epsilon_{0}\right)}{\partial W} W_{0}+\frac{\delta}{2} \frac{\partial U_{B}\left(0, W_{0}, 1 / \epsilon_{0}\right)}{\partial W} W_{0}\right) \\
\frac{\partial \hat{V}_{G}\left(0, W_{0}, \epsilon_{0}\right)}{\partial \epsilon} \epsilon^{\prime}(0) & =\left(\frac{1}{1-\delta}\right)\left(\left(1-\frac{\delta}{2}\right) \frac{\partial U_{G}\left(0, W_{0}, \epsilon_{0}\right)}{\partial \varepsilon} \epsilon^{\prime}(0)-\left(\frac{\delta}{2}\right) \frac{\partial U_{B}\left(0, W_{0}, 1 / \epsilon_{0}\right)}{\partial \varepsilon}\left(\frac{\epsilon^{\prime}(0)}{\epsilon_{0}^{2}}\right)\right) .
\end{aligned}
$$

It is confirmed below that $\partial U_{G}\left(0, W_{0}, \epsilon_{0}\right) / \partial T>0$ and $\partial U_{G}\left(0, W_{0}, \epsilon_{0}\right) / \partial \varepsilon<0$. Therefore, for $V_{G}^{\prime}(0)>0$, we require $\delta>\underline{\delta}^{f}$ where

$$
\frac{1}{\underline{\delta}^{f}}=\frac{\frac{1}{2}\left(\frac{\partial U_{B}\left(0, W_{0}, 1 / \epsilon_{0}\right)}{\partial T}+\frac{\partial U_{G}\left(0, W_{0}, \epsilon_{0}\right)}{\partial T} \epsilon_{0}-\left(\frac{\partial U_{B}\left(0, W_{0}, 1 / \epsilon_{0}\right)}{\partial \varepsilon}\left(\frac{1}{\epsilon_{0}^{2}}\right)+\frac{\partial U_{G}\left(0, W_{0}, \epsilon_{0}\right)}{\partial \varepsilon}\right) \epsilon^{\prime}(0)+\left(\frac{W^{\prime}(0)}{W_{0}}\right)\left(\frac{\partial U_{G}\left(0, W_{0}, \epsilon_{0}\right)}{\partial W} W_{0}+\frac{\partial U_{B}\left(0, W_{0}, 1 / \epsilon_{0}\right)}{\partial W} W_{0}\right)\right)}{\frac{\partial U_{G}\left(0, W_{0}, \epsilon_{0}\right)}{\partial T} \epsilon_{0}-\frac{\partial U_{G}\left(0, W_{0}, \epsilon_{0}\right)}{\partial \varepsilon} \epsilon^{\prime}(0)} .
$$

This is equation (D.5) in Appendix D. To evaluate this, we evaluate the component elements and substitute into (S.5). First, we have

$$
U_{G}(-\epsilon(\mathcal{T}) \mathcal{T}, W, \epsilon)=\frac{X_{G}(W, \epsilon)^{1-\gamma}}{1-\gamma}-\frac{L(-\epsilon(\mathcal{T}) \mathcal{T}, W)^{1+\psi}}{1+\psi}
$$

and

$$
U_{B}(\mathcal{T}, W, 1 / \epsilon)=\frac{X_{B}(W, 1 / \epsilon)^{1-\gamma}}{1-\gamma}-\frac{L(\mathcal{T}, W)^{1+\psi}}{1+\psi}
$$


where labour supply is given by $L(T, W)=\rho(1-T) / W$. To find the expression for consumption let

$$
A=\left(a_{G}^{1-\sigma}+a_{B}^{1-\sigma}\right)^{\frac{1}{1-\sigma}} \quad \text { and } \quad B(\epsilon):=B_{G}(\epsilon)=\left(\frac{1}{2} b_{G}+\frac{1}{2} b_{B} \varepsilon^{1-\sigma}\right)^{\frac{1}{1-\sigma}} .
$$

With this definition $B_{B}(1 / \epsilon)=B(\epsilon) / \epsilon$. For convenience, let $\tilde{X}_{B}(W, \epsilon):=X_{B}(W, 1 / \epsilon)$. Then consumption is given by

$$
X_{G}(W, \epsilon)=\xi \mu^{-1} \frac{1}{\frac{W}{\rho} A B(\varepsilon)} \quad \text { and } \quad \tilde{X}_{B}(W, \epsilon)=\xi \mu^{-1} \frac{1}{\frac{W}{\rho} A \frac{B(\epsilon)}{\epsilon}}=\epsilon X_{G}(W, \epsilon) .
$$

From these equations we get

$$
\frac{\partial X_{G}(W, \epsilon)}{\partial \epsilon}=-X_{G}(W, \epsilon) \frac{B^{\prime}(\epsilon)}{B(\epsilon)} \quad \text { and } \quad \frac{\partial \tilde{X}_{B}(W, \epsilon)}{\partial \epsilon}=-\tilde{X}_{B}(W, \epsilon)\left(\frac{1}{\epsilon}\right)\left(\epsilon \frac{B^{\prime}(\epsilon)}{B(\epsilon)}-1\right) .
$$

Also,

$$
\frac{\partial L(T, W)}{\partial T}=-\frac{\rho}{W} ; \quad W \frac{\partial L(T, W)}{\partial W}=-L(T, W)
$$

and

$$
W \frac{\partial X_{G}(W, \epsilon)}{\partial W}=-X_{G}(W, \epsilon) ; \quad W \frac{\partial \tilde{X}_{B}(W, \epsilon)}{\partial W}=-\tilde{X}_{B}(W, \epsilon)
$$

We have

$$
\frac{\partial U_{G}\left(0, W_{0}, \epsilon_{0}\right)}{\partial T}=\frac{\partial U_{B}\left(0, W_{0}, 1 / \epsilon_{0}\right)}{\partial T}=\left(\frac{W_{0}}{\rho}\right)^{-(1+\psi)}
$$

and

$$
\begin{aligned}
W_{0} \frac{\partial U_{G}\left(0, W_{0}, \epsilon_{0}\right)}{\partial W} & =W_{0} X_{G}\left(W_{0}, \epsilon_{0}\right)^{-\gamma} \frac{\partial X_{G}\left(W_{0}, \epsilon_{0}\right)}{\partial W}-W_{0} L\left(0, W_{0}\right)^{\psi} \frac{\partial L\left(0, W_{0}\right)}{\partial W} \\
& =-X_{G}\left(W_{0}, \epsilon_{0}\right)^{1-\gamma}+L\left(0, W_{0}\right)^{1+\psi} \\
& =-\xi^{1-\gamma} A^{\gamma-1} B\left(\epsilon_{0}\right)^{\gamma-1} \mu^{\gamma-1}\left(\frac{W_{0}}{\rho}\right)^{\gamma-1}+\left(\frac{W_{0}}{\rho}\right)^{-(1+\psi)} \\
& =\left(\frac{W_{0}}{\rho}\right)^{-(1+\psi)}\left(-\xi^{1-\gamma} A^{\gamma-1} B\left(\varepsilon_{0}\right)^{\gamma-1} \mu^{\gamma-1}\left(\frac{\rho}{W_{0}}\right)^{-\gamma-\psi}+1\right) \\
& =\left(\frac{W_{0}}{\rho}\right)^{-(1+\psi)}\left(-\kappa_{2} \frac{B\left(\varepsilon_{0}\right)^{\gamma-1}}{\frac{1}{2} B\left(\varepsilon_{0}\right)^{\gamma-1}+\frac{1}{2}\left(\frac{B\left(\varepsilon_{0}\right)}{\varepsilon_{0}}\right)^{\gamma-1}}+1\right)
\end{aligned}
$$

where the last line uses (A.9) with $T_{s}=0$ for $s=G, B, A_{s}=A$ and $\mathrm{E}\left[B_{s}\left(\varepsilon_{s}(0)\right)\right]=(1 / 2) B\left(\epsilon_{0}\right)^{\gamma-1}+$ $\left.(1 / 2)\left(B\left(\epsilon_{0}\right)\right) / \epsilon_{0}\right)^{\gamma-1}$. Let $\tilde{U}_{B}(T, W, \epsilon):=U_{B}(T, W, 1 / \epsilon)$. Then, similarly

$$
\begin{aligned}
W_{0} \frac{\partial \tilde{U}_{B}\left(0, W_{0}, \epsilon_{0}\right)}{\partial W} & =-\tilde{X}_{B}\left(W_{0}, \epsilon_{0}\right)^{1-\gamma}+L\left(0, W_{0}\right)^{1+\psi} \\
& =-\epsilon_{0}^{1-\gamma} \xi^{1-\gamma} A^{\gamma-1} B\left(\varepsilon_{0}\right)^{\gamma-1} \mu^{\gamma-1}\left(\frac{W_{0}}{\rho}\right)^{\gamma-1}+\left(\frac{W_{0}}{\rho}\right)^{-(1+\psi)} \\
& =\left(\frac{W_{0}}{\rho}\right)^{-(1+\psi)}\left(-\xi^{1-\gamma} A^{\gamma-1}\left(\frac{B\left(\varepsilon_{0}\right)}{\varepsilon_{0}}\right)^{\gamma-1} \mu^{\gamma-1}\left(\frac{W_{0}}{\rho}\right)^{\gamma+\psi}+1\right) \\
& =\left(\frac{W_{0}}{\rho}\right)^{-(1+\psi)}\left(-\kappa_{2} \frac{\left(\frac{B\left(\varepsilon_{0}\right)}{\varepsilon_{0}}\right)^{\gamma-1}}{\frac{1}{2} B\left(\varepsilon_{0}\right)^{\gamma-1}+\frac{1}{2}\left(\frac{B\left(\varepsilon_{0}\right)}{\varepsilon_{0}}\right)^{\gamma-1}}+1\right) .
\end{aligned}
$$


Hence, since $\partial \tilde{U}_{B}(T, W, \epsilon) / \partial W=\partial U_{B}(T, W, 1 / \epsilon) / \partial W$

$$
\frac{1}{2} \frac{\partial U_{G}\left(0, W_{0}, \epsilon_{0}\right)}{\partial W} W_{0}+\frac{1}{2} \frac{\partial U_{B}\left(0, W_{0}, 1 / \epsilon_{0}\right)}{\partial W} W_{0}=-\left(\frac{W_{0}}{\rho}\right)^{-(1+\psi)}\left(\kappa_{2}-1\right) .
$$

Recalling that $\epsilon_{0}=\left(b_{G} / b_{B}\right)^{-1 / \sigma}$ and differentiating $B(\epsilon)$ we get

$$
\frac{\epsilon B^{\prime}(\epsilon)}{B(\epsilon)}=\frac{1}{1+\epsilon^{\sigma-1} \epsilon_{0}^{-\sigma}}
$$

Evaluating at $\epsilon_{0}$ gives

$$
\frac{\epsilon_{0} B^{\prime}\left(\epsilon_{0}\right)}{B\left(\epsilon_{0}\right)}=\frac{\epsilon_{0}}{1+\epsilon_{0}} \quad \text { and } \quad \frac{\epsilon_{0} B^{\prime}\left(\epsilon_{0}\right)}{B\left(\epsilon_{0}\right)}-1=-\frac{1}{1+\epsilon_{0}} .
$$

Next, repeating equation $(21)$

$$
\epsilon(\mathcal{T})=\left(\frac{b_{G}}{b_{B}}\right)^{-\frac{1}{\sigma}}\left(\frac{1+\epsilon(\mathcal{T}) \mathcal{T}}{1-\mathcal{T}}\right)^{\frac{1}{\sigma}}
$$

where $\mathcal{T}$ is the transfer received by the country with the bad productivity shock. Differentiating implicity we get

$$
\epsilon^{\prime}(\mathcal{T})=\frac{\epsilon(\mathcal{T})\left(\varepsilon_{0}^{\sigma}+\epsilon(\mathcal{T})^{\sigma-1}\right)}{\sigma \epsilon(\mathcal{T})^{\sigma-1}-\mathcal{T}\left(\epsilon_{0}^{\sigma}+\sigma \epsilon(\mathcal{T})^{\sigma-1}\right)}=\frac{\epsilon(\mathcal{T})(1+\epsilon(\mathcal{T}))}{(1-\mathcal{T})(\sigma(1+\mathcal{T} \epsilon(\mathcal{T}))-\mathcal{T} \epsilon(\mathcal{T}))}
$$

Evaluating at $\mathcal{T}=0$ gives

$$
\frac{\epsilon^{\prime}(0)}{\epsilon_{0}}=\frac{\left(1+\epsilon_{0}\right)}{\sigma}
$$

Define

$$
E B_{\gamma}\left(\epsilon_{0}\right):=\left(\frac{1}{2} B\left(\epsilon_{0}\right)^{\gamma-1}+\frac{1}{2}\left(\frac{B\left(\epsilon_{0}\right)}{\epsilon_{0}}\right)^{\gamma-1}\right)
$$

Hence, differentiating with respect to $\epsilon$ gives

$$
\begin{aligned}
\frac{\partial U_{G}\left(0, W_{0}, \epsilon_{0}\right)}{\partial \varepsilon} \epsilon^{\prime}(0) & =X_{G}\left(W_{0}, \epsilon_{0}\right)^{-\gamma} \frac{\partial X_{G}\left(W_{0}, \epsilon_{0}\right)}{\partial \varepsilon} \epsilon^{\prime}(0) \\
& =-X_{G}\left(W_{0}, \epsilon_{0}\right)^{1-\gamma}\left(\frac{B^{\prime}\left(\varepsilon_{0}\right)}{B\left(\epsilon_{0}\right)}\right) \epsilon^{\prime}(0)=-\left(\frac{\epsilon_{0}}{\sigma}\right) X_{G}\left(W_{0}, \epsilon_{0}\right)^{1-\gamma} \\
& =-\left(\frac{\epsilon_{0}}{\sigma}\right) \xi^{1-\gamma} \mu^{\gamma-1} A^{\gamma-1}\left(\frac{W_{0}}{\rho}\right)^{\gamma-1} B\left(\epsilon_{0}\right)^{\gamma-1} \\
& =-\left(\frac{W_{0}}{\rho}\right)^{-(1+\psi)}\left(\left(\frac{\epsilon_{0}}{\sigma}\right) \xi^{1-\gamma} \mu^{\gamma-1} A^{\gamma-1}\left(\frac{W_{0}}{\rho}\right)^{\gamma+\psi} B\left(\epsilon_{0}\right)^{\gamma-1}\right) \\
& =-\left(\frac{W_{0}}{\rho}\right)^{-(1+\psi)}\left(\frac{\kappa_{2} \epsilon_{0}}{\sigma}\right)\left(\frac{B\left(\epsilon_{0}\right)^{\gamma-1}}{E B_{\gamma}\left(\epsilon_{0}\right)}\right)
\end{aligned}
$$

where the second line substitutes for $B^{\prime}\left(\epsilon_{0}\right)$ and $\epsilon^{\prime}(0)$ and again use has been made of equation (A.9) in the 
last line. Similarly

$$
\begin{aligned}
\frac{\partial \tilde{U}_{B}\left(0, W_{0}, \epsilon_{0}\right)}{\partial \epsilon} \epsilon^{\prime}(0) & =\tilde{X}_{B}\left(W_{0}, \epsilon_{0}\right)^{-\gamma} \frac{\partial \tilde{X}_{B}\left(W_{0}, \epsilon_{0}\right)}{\partial \epsilon} \epsilon^{\prime}(0) \\
& =-\tilde{X}_{B}\left(W_{0}, \epsilon_{0}\right)^{1-\gamma}\left(\frac{\epsilon_{0} B^{\prime}\left(\epsilon_{0}\right)}{B\left(\epsilon_{0}\right)}-1\right)\left(\frac{\epsilon^{\prime}(0)}{\epsilon_{0}}\right)=\left(\frac{1}{\sigma}\right) \tilde{X}_{B}\left(W_{0}, \epsilon_{0}\right)^{1-\gamma} \\
& =\left(\frac{1}{\sigma}\right) \xi^{1-\gamma} \mu^{\gamma-1} A^{\gamma-1}\left(\frac{W_{0}}{\rho}\right)^{\gamma-1}\left(\frac{B\left(\epsilon_{0}\right)}{\epsilon_{0}}\right)^{\gamma-1} \\
& =\left(\frac{W_{0}}{\rho}\right)^{-(1+\psi)}\left(\left(\frac{1}{\sigma}\right) \xi^{1-\gamma} \mu^{\gamma-1} A^{\gamma-1}\left(\frac{\rho}{W_{0}}\right)^{-\gamma-\psi}\left(\frac{B\left(\epsilon_{0}\right)}{\epsilon_{0}}\right)^{\gamma-1}\right) \\
& =\left(\frac{W_{0}}{\rho}\right)^{-(1+\psi)}\left(\frac{\kappa_{2}}{\sigma}\right)\left(\frac{\left(\frac{B\left(\epsilon_{0}\right)}{\epsilon_{0}}\right)^{\gamma-1}}{E B_{\gamma}\left(\epsilon_{0}\right)}\right)
\end{aligned}
$$

Since $\partial \tilde{U}_{B}\left(0, W_{0}, \epsilon_{0}\right) / \partial \epsilon=-\left(1 / \epsilon_{0}^{2}\right) \partial U_{B}\left(0, W_{0}, 1 / \epsilon_{0}\right) / \partial \varepsilon$, substituting terms into (S.5) gives

$$
\frac{1}{\underline{\delta}^{f}}=\frac{\frac{1}{2}\left(1+\epsilon_{0}\right)+\frac{1}{2}\left(\frac{\kappa_{2}}{\sigma}\right)\left(\frac{\left(\frac{B\left(\epsilon_{0}\right)}{\epsilon_{0}}\right)^{\gamma-1}}{E B_{\gamma}\left(\epsilon_{0}\right)}\right)+\frac{1}{2}\left(\frac{\kappa_{2} \epsilon_{0}}{\sigma}\right)\left(\frac{\left(B\left(\epsilon_{0}\right)\right)^{\gamma-1}}{E B_{\gamma}\left(\epsilon_{0}\right)}\right)-\left(\kappa_{2}-1\right)\left(\frac{W^{\prime}(0)}{W_{0}}\right)}{\epsilon_{0}+\left(\frac{\kappa_{2} \epsilon_{0}}{\sigma}\right)\left(\frac{\left(B\left(\epsilon_{0}\right)\right)^{\gamma-1}}{E B_{\gamma}\left(\epsilon_{0}\right)}\right)}
$$

Next, to calculate $W^{\prime}(0) / W_{0}$, use the wage equation (17),

$$
(W(\mathcal{T}))^{\gamma+\psi}=\frac{\kappa_{0} A^{1-\gamma}\left((1+\epsilon(\mathcal{T}) \mathcal{T})^{1+\psi}+(1-\mathcal{T})^{1+\psi}\right)}{(B(\epsilon(\mathcal{T})))^{\gamma-1}(1+\epsilon(\mathcal{T}) \mathcal{T})+\left(\frac{B(\epsilon(\mathcal{T}))}{\epsilon(\mathcal{T})}\right)^{\gamma-1}(1-\mathcal{T})}
$$

Differentiating and evaluating at $\mathcal{T}=0$, and using equation (S.7) gives (after some manipulation)

$$
\begin{aligned}
(\gamma+\psi) \frac{W^{\prime}(0)}{W_{0}}= & \frac{(1+\psi)}{2}\left(\epsilon_{0}-1\right) \\
& -\frac{1}{2} \frac{B\left(\epsilon_{0}\right)^{\gamma-1} \epsilon_{0}\left(1+(\gamma-1)\left(\frac{B^{\prime}\left(\epsilon_{0}\right)}{B\left(\epsilon_{0}\right)}\right)\left(\frac{\epsilon^{\prime}(0)}{\epsilon_{0}}\right)\right)}{E B_{\gamma}\left(\varepsilon_{0}\right)} \\
& +\frac{1}{2} \frac{\left(\frac{B\left(\epsilon_{0}\right)}{\epsilon_{0}}\right)^{\gamma-1}\left(1-(\gamma-1)\left(\frac{\epsilon_{0} B^{\prime}\left(\epsilon_{0}\right)}{B\left(\epsilon_{0}\right)}-1\right)\left(\frac{\epsilon^{\prime}(0)}{\epsilon_{0}}\right)\right)}{E B_{\gamma}\left(\epsilon_{0}\right)}
\end{aligned}
$$

Using equations (S.6) and (S.7) gives

$$
\frac{W^{\prime}(0)}{W_{0}}=\left(\frac{1}{(\gamma+\psi)}\right)\left(\frac{(1+\psi)}{2}\left(\epsilon_{0}-1\right)-\left(\frac{1}{2} \frac{B\left(\epsilon_{0}\right)^{\gamma-1} \epsilon_{0}\left(1+\frac{\gamma-1}{\sigma}\right)}{E B_{\gamma}\left(\varepsilon_{0}\right)}-\frac{1}{2} \frac{\left(\frac{B\left(\epsilon_{0}\right)}{\epsilon_{0}}\right)^{\gamma-1}\left(1+\frac{\gamma-1}{\sigma}\right)}{E B_{\gamma}\left(\epsilon_{0}\right)}\right)\right) .
$$

Taking approximations in (S.8) and (S.9) about $z=1$ gives

$$
\frac{W^{\prime}(0)}{W_{0}} \approx-\frac{1}{2}\left(\frac{1}{\gamma+\psi}\right)\left((\gamma-1)\left(1+\frac{\gamma}{\sigma}\right)-\psi\right) \frac{(\sigma-1)}{\sigma}(z-1)
$$

and

$$
\frac{1}{\underline{\delta}^{f}} \approx 1+\frac{1}{2}\left(\frac{1}{\kappa_{2}+\sigma}\right)\left(\frac{1}{\gamma+\psi}\right)\left(\sigma\left(\kappa_{2}-1\right)\left((\gamma-1)\left(1+\frac{\gamma}{\sigma}\right)-\psi\right)-(\gamma+\psi)\left(\kappa_{2} \gamma+\sigma\right)\right)\left(\frac{\sigma-1}{\sigma}\right)(z-1)
$$


Then, using $1 / \kappa_{2}=\mu(\theta-1)(\sigma-1) / \theta \sigma$ gives

$\frac{1}{\underline{\delta}^{f}} \approx 1+\frac{1}{2}\left(\frac{\left((\gamma-1)\left(1+\frac{\gamma}{\sigma}\right)-\psi\right)\left(\sigma-\mu(\sigma-1)\left(\frac{\theta-1}{\theta}\right)\right)-(\gamma+\psi)\left(\gamma+\mu(\sigma-1)\left(\frac{\theta-1}{\theta}\right)\right)}{(\gamma+\psi)\left(1+\mu(\sigma-1)\left(\frac{\theta-1}{\theta}\right)\right)}\right)\left(\frac{\sigma-1}{\sigma}\right)(z-1)$

Using $\underline{\lambda}:=(\underline{\delta}-1) / \underline{\delta}$ and the approximation $\underline{\delta} \approx 1+\underline{\lambda}$ and setting $\mu=\psi=(\theta-1) / \theta=1$ gives $(28)$ in the text. 